
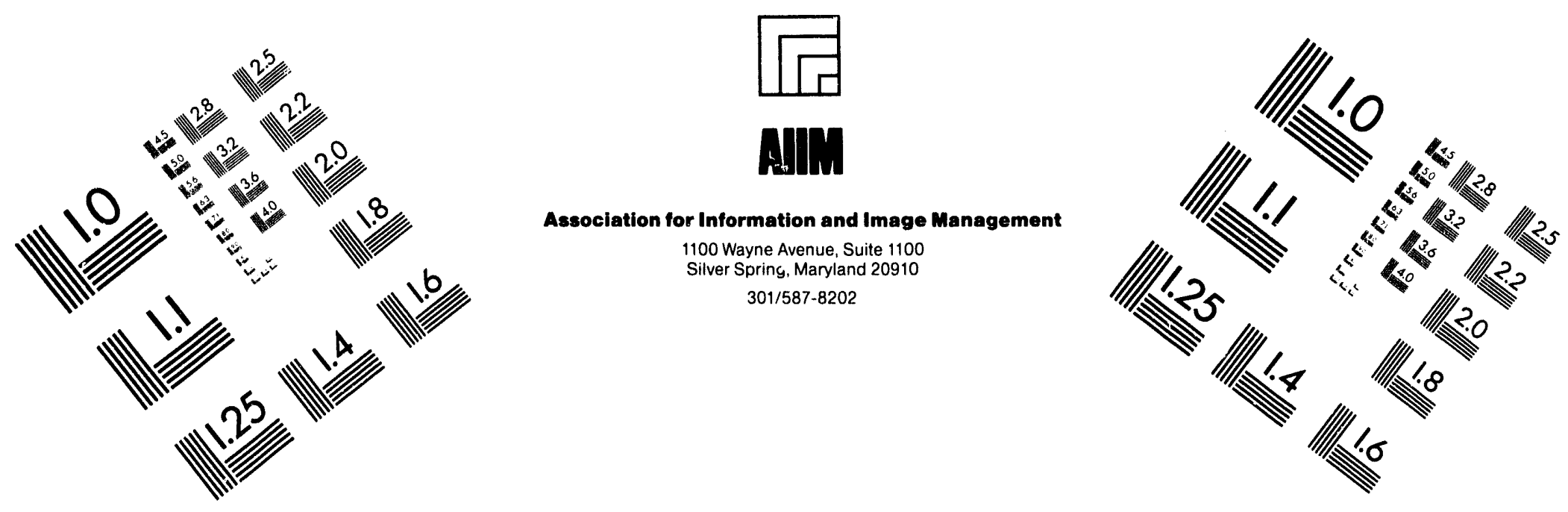

\title{
Centimeter
}

2
1

Inches
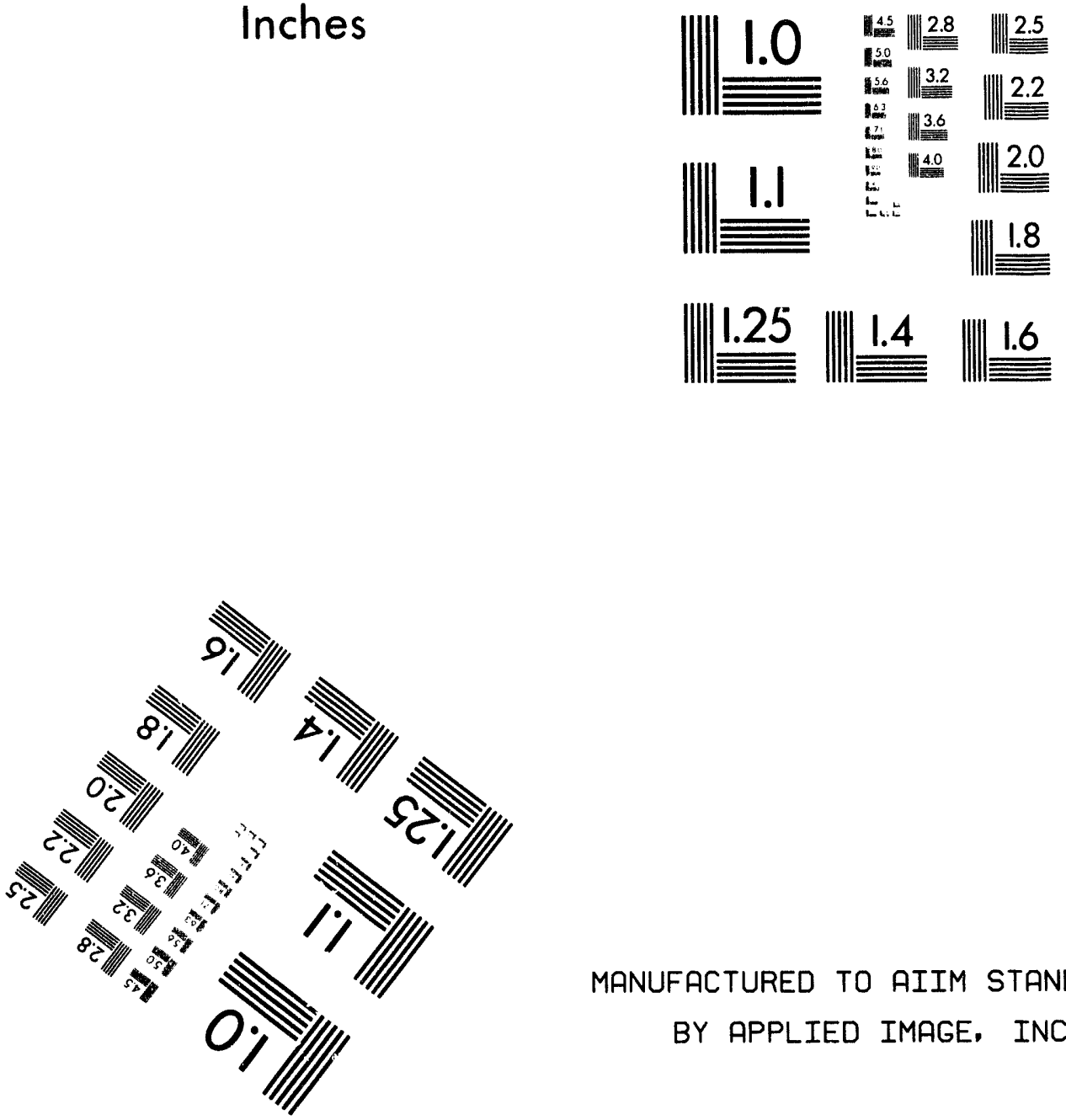

MANUFACTURED TO AIIM STANDARDS

BY APPLIED IMAGE, INC.

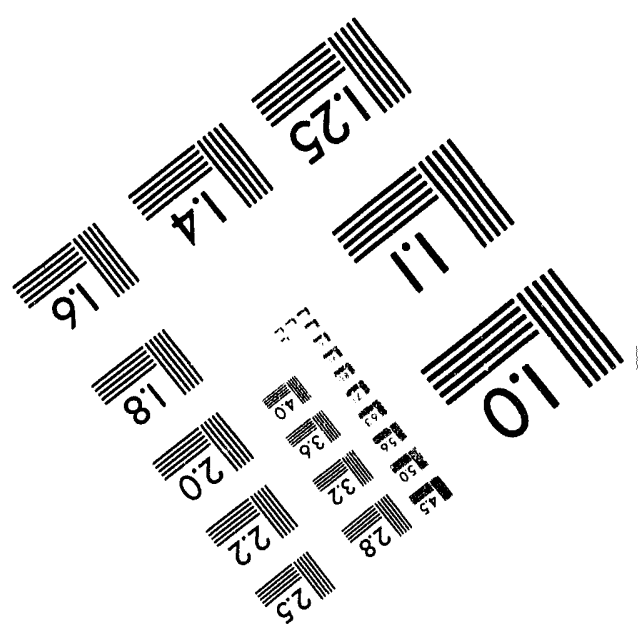



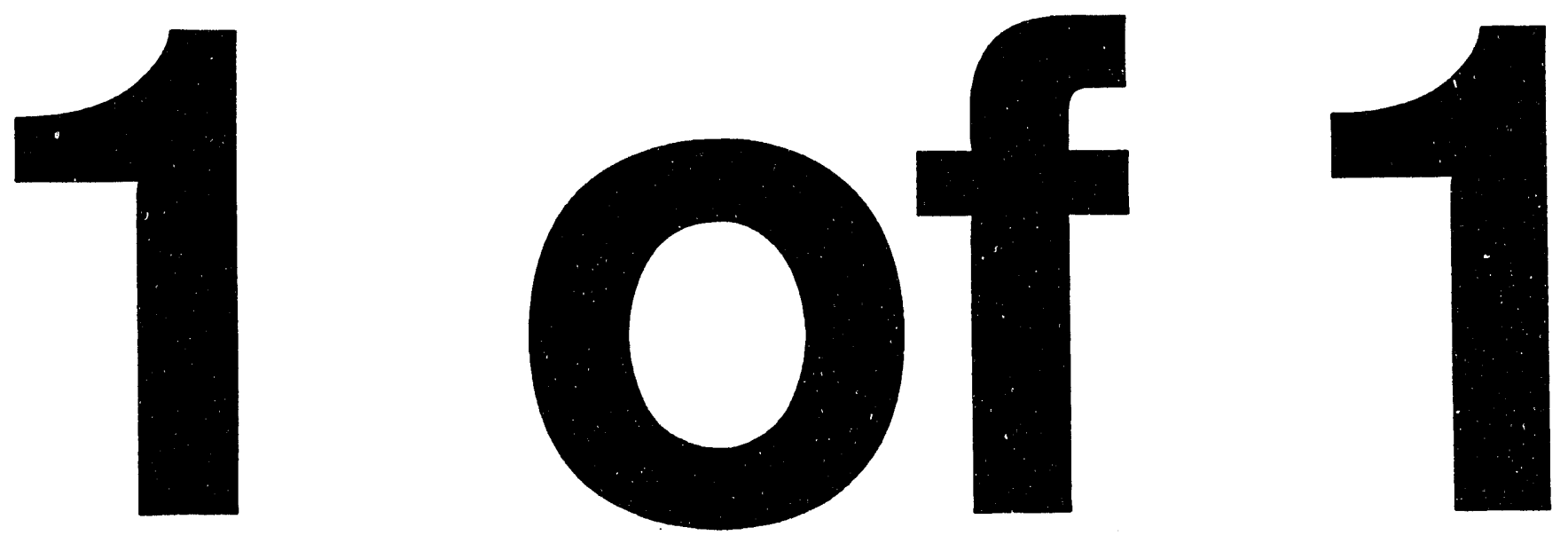


\section{ELEMENTARY PARTICLE PHYSICS AT THE UNIVERSITY OF FLORIDA}

\section{Annual Progress Report \\ DOE GRANT DE-FG05-86ER40272}

University of Florida

Gainesville, Florida 32611

November 1, 1999

Task A: Theoretical Elementary Particle Physics

R. Field, P. Ramond, P. Sikivie, C. Thorn

Task B: Experimental Elementary Particle Physics

P. Avery and J. Yelton

Task C: Axion Project

P. Sikivie, N. Sullivan, D. Tanner

Task S: Computer Acquisition

P. Avery and J. Yelton

Grant Coordinator: R. D. Field 
TASK A

\title{
RESEARCH IN THEORETICAL ELEMENTARY PARTICLE PHYSICS
}

\section{AT THE UNIVERSITY OF FLORIDA}

\author{
Annual Progress Report \\ DOE GRANT DE-FG05-86ER40272
}

University of Florida

Gainesville, Florida 32611

November 1, 1993

\author{
Principal Investigators: \\ Richard D. Field \\ Pierre M. Ramond \\ Pierre Sikivie \\ Charles B. Thorn
}

\section{DISCLAIMER}

Grant Spokesperson: R. D. Field

This report was prepared as an account of work sponsored by an agency of the United States Government. Neither the United States Government nor any agency thereof, nor any of their employees, makes any warranty, express or implied, or assumes any legal liability or recponsibility for the accuracy, completeness, or usefulness of any information, apparatus, product, or process disclosed, or represents that its use would not infringe privately uwned rights. Reference herein to any specific commercial product, process, or service by trade name, trademark, manufacturer, or otherwise does not necessarily constitute or imply its endorsement, recommendation, or favoring by the United States Government or any agency thereof. The views and opinions of authors expressed herein do not necessarily state or reflect those of the United States Government or any agency thereof. 


\title{
Annual Progress Report TASK A
}

\author{
Theoretical Elementary Particle Research at the \\ University of Florida \\ Principal Investigators: \\ Richard D. Field \\ Pierre M. Ramond \\ Pierre Sikivie \\ Charles B. Thorn
}

\begin{abstract}
This is the Annual Progress Report of the theoretical particle theory group at the University of Florida under DoE Grant DE-FG05-86ER40272. At present our group consists of four Full Professors (Field, Ramond, Thorn, Sikivie) and three Assistant Professors (Qiu, Woodard, Kennedy). Dallas Kennedy recently joined our group increasing the Particle Theory faculty to seven. In addition, we have three postdoctoral research associates, an SSC fellow, and eight graduate students. The research of our group covers a broad range of topics in theoretical high energy physics with balance between theory and phenomenology. Included in this report is a summary of the last several years of operation of the group and an outline of our current research program.
\end{abstract}

\section{OUTLINE}

I. Introduction

II. History of the Particle Theory Group

III. Institute for Fundamental Theory (IFT)

IV. Particle Theory Personnel
(a) Faculty
(b) Postdocs
(c) Graduate Students

V. Scientific Statements of the Group Members

VI. Activities of the Particle Theory Group

Appendix A. Group Publications 


\section{INTRODUCTION}

This progress report on theoretical elementary particle physics is presented by the Particle Theory Group at the University of Florida. The Particle Theory group is part of the interdisciplinary Institute for Fundamental Theory (IFT), an institute solely funded by the University. The history of the Theory Group is presented in section II, followed by a discussion of the IFT in section III.

At present our group consists of four Full Professors, R. D. Field, P. Ramond, C. Thorn, and P. Sikivie and three Assistant Professors, D. Kennedy, Z. Qiu and R. Woodard. Dallas Kennedy has just joined our faculty, effective August 1993. In addition, we have three postdoctoral research associates funded by the DoE, one SSC postdoctoral fellow, and a number of graduate students. We have produced at least one Ph.D. each of the last six years. More details about the graduate students are given in section IV.

The scientific activities of our group are rich and varied, ranging from theoretical theory all the way to numerical phenomenology. At one end of the spectrum, the baffling conceptual theoretical problem of formulating a consistent quantum theory of gravity is one aspect of the research conducted here. The extraordinarily rich superstring theory as the only known consistent theory of quantum gravity also provides a theoretical laboratory in which this difficult subject can be studied. Further, string theory offers new directions of study and links between hitherto unrelated fields of study. Some of these directions are explored in research conducted here. Although many believe that string theory will link the experimentally remote Planck world with the accessible world of particle accelerators and other detectors, this has not yet been demonstrated. The low energy world is at present in a bizarre theoretical state as it is marvelously described by the Standard Model. No deviations from this model have yet been found, but it is widely believed that it is not fundamental but rather the chiral shard of a more integrated structure, perhaps string theory. In the meantime, the question of what lies ' ectly beyond the standard model is being reinvestigated by our group, providing signatures for the structures that lie just beyond the grasp of experimentalists. Although the main theoretical extensions have been formulated a decade ago, they must be reexamined in the light of the better quality of the available data. Our group is deeply involved in one particular aspect of this work, the supersymmetric minimal extension of the standard model. Astrophysics also provides strong hints about the shortcomings of the standard model: many astrophysical phenomena, such as high energy cosmic rays, galaxy formation, and perhaps solar neutrinos and dark matter, are yet unexplained by the standard model. There also, the quality of the data has shown spectacular improvement. Our group is active in this area, both on the theoretical side and in the devising of new types of detectors for dark matter. Finally, the important question of detectability of new physics in colliders is addressed by the research of our group. This can only happen if the new physics signatures can be extracted from the ever-present standard model background. It is crucial to know which series of cuts have to be made to minimize the background and expose the new phenomena we all hope will emerge at the new accelerators. This computer intensive study is conducted in contact with experimentalists. Thus we feel that our group's activities correctly reflect many of the modern trends in our field. The detailed research conducted by the group 
is presented through individual's statements in section $\mathrm{V}$ with a list of publications in Appendix A.

In view of the geographical isolation of our campus, it is essential to the scientific survival and thriving of our group that we maintain a vigorous seminar and visitor program. Equally important is the ability for the members of our group to travel and collaborate with colleagues at other high energy physics universities and laboratories. The activities of the theory group center around a weekly seminar by an outside speaker, and a weekly journal club with an internal speaker. In addition, a small number of longer term visits to the group are made possible through the IFT. In section VI we provide a list of last year's visitors as well as the professional activities of the group members.

Within the last few years our group has shown improvement in several areas. One is through the hiring of three Assistant Professors in different areas of theoretical particle physics. Our most recent hire, Dallas Kennedy, is an expert in the radiative corrections of the standard model, and his presence here underlies our commitment to maintaining a close contact with the experimental world. Another is through the quality of our postdocs, whom we have successfully placed in academic jobs (postdocs or junior faculty); the third is through the recent crop of excellent graduate stucients. There also we have had excellent success in placing our students in research postdoctoral positions at academic institutions.

In each of the last five years, we have awarded at least one Ph.D., and placed our students in research postdocs. More are expected to graduate in the coming years, and many are actively collaborating with members of the group. We feel that the training students in theoretical physics is an important part of our role. Each year we send students to TASI and other summer programs. 


\section{HISTORICAL SURVEY}

The high energy theory group at the University of Florida was created in 1980 with the arrival of T. Curtright, R. D. Field, P. Ramond, and C. Thorn. The following year we were successful in acquiring a DOE grant. T. Curtright received a DOE Junior Investigator award. They were joined in 1981 by P. Sikivie. At that time the group had two postdoctoral associates, one funded by DOE and the other by the Department of Physics. In 1987 , in response to the formation of the IFT, the group was allowed to increase by two junior faculty. These positions were filled by Z. Qiu in 1988 and R. Woodard in 1989. T. Curtright left the group in 1986 to join the faculty at the University of Miami. This year we hired Dallas Kennedy from Fermilab to fill Curtright's position and to bring the size of the group to seven faculty.

Two members of our group have received Guggenheim fellowships. Members of our group have served on the Fermilab PAC and on HEPAP.

Over the last ten years we have had excellent postdoctoral research associates. We have had as many as six concurrent postdoctoral positions. At that time two were funded by the Department of Physics, one by IFT and three by DoE. However, at present, the Department of Physics will no longer provide for postdoctoral research associates and we are down to just three postdocs (all supported by DoE). Furthermore, since 1987 we have been producing a steady stream of good graduate students.

The rise of our group is but one aspect of the growth at the University of Florida. Over the last ten years, the Department of Physics at the University of Florida has more than doubled in size. In addition to the growth of the high energy theory group four faculty were hired in the area of theoretical astrophysics (Ipser, Detweiler, Fry, Whiting), one in the area of mathematical physics (Klauder), and six in condensed matter theory (Hershfield, Hirschfeld, Ingersent, Muttalib, Obukhov, Stanton). Schrieffer joined our Department, jointly with Florida State, on January 1, 1992. We have fruitful interactions with these groups through the Institute for Fundamental Theory (IFT). It has been more difficult to attract experimental high energy physics to Florida. However, we have succeeded in hiring three faculty in high energy experimental physics (Walker, Avery, Yelton). We currently have a search underway for an additional senior experimentalist and we hope to hire several experimental faculty in collider physics. The increase in size and quality of the science personnel in Florida was recognized recently when the NSF awarded the Magnet Lab to Florida State and the University of Florida.

From 1980 to 1990, the Department of Physics at the University of Florida hired, on the average, about three new faculty per year. The high energy theory group has succeeded because of the conscientious efforts of the faculty and postdoctoral associates and because of the financial support we have received from the Department of Physics, the University of Florida, and the Department of Energy. 


\section{INSTITUTE FOR FUNDAMENTAL THEORY}

The particle theory group has benefited substantially from the creation of the Institute for Fundamental Theory (IFT) at the University of Florida. The IFT is an interdisciplinary center involving high energy theory (HET), condensed matter theory (CMT), cosmology-astrophysics, and mathematics and receives operating funds from the University of Florida. The aim of the IFT is to provide the right intellectual environment for attracting the brightest among young mathematicians and theoretical physicists. Our group has benefited directly from the improved environment provided by IFT. In addition, the IFT has contributed funds for high energy theory visitors and provides the salary for one of our particle theory postdoctoral research associates. Furthermore, the creation of the IFT was responsible for our sixth and seventh faculty position in particle theory.

We believe that in the years to come young theorists will tend to thrive in a mul tidisciplinary environment which includes close interaction between mathematicians and physicists. There are several reasons for this belief. First of all in physics there has been in the past ten years a dramatic confluence between hitherto independent discipline: namely, cosmology-astrophysics, particle physics, and condensed matter physics.

The marriage between cosmology and HET is well documented, and needs no elaboration here. So far the links between HET and CMT have occurred along the lines of quantum statistical mechanics, quantum field theory, critical phenomena, and there has also been dramatic overlap between CMT and cosmology via the study of the kinetics of phase transition. In our view the overlap between CMT and HET will increase still more along the lines of deriving specific mechanisms of symmetry breaking. In particular it is hoped that the techniques in string theory might prove useful to the solution of $\mathrm{CMT}$ problems. In addition with the advent of string theory there is a need for a rapport between gravitation and HET, in order to arrive at a geometrical formulation of string gravity.

Just as there has been a tightening of the bonds between different areas of theoretical physics due to the universality of the methods used, their mathematical origins have motivated a historic rapprochement with pure mathematics. Thus the rationales for the IFT.

The intellectual environment of the High Energy Theory group has been enhanced by the IFT, as it brings distinguished visitors in closely related disciplines. In the recent years the IFT has funded long term visits to the group by people such as Witten, Coleman, Singer, Brink, Goddard, Hartle, and Whightman.

The first Workshop sponsored by the Institute for Fundamental Theory was held on the campus of the University of Florida on February 14 to 16 1992. Its central theme was "Dark Matter". The purpose of the workshop was to assemble observers and theorists to discuss the various questions associated with dark matter, its nature, its abundance, and its detection. At the workshop, observers and theorists discussed these questions and many more, including novel methods of detection, and new analyses of peculiar velocities that seem to indicate the pervasive nature of dark matter. The proceedings summarize these contributions and will appear shortly. 
The success of the first workshop has motivated a second IFT workshop to be held in February 1994. This second workshop will focus on "Yukawa Couplings and their Origins". We are asking DoE for some support for the workshop.

In addition, the IFT has an external advisory board. The original board members were E. Abrahams, D. Eardly, Y. Nambu, and I. Singer. The current board consists of E. Abrahams and D. Eardly together with Ken Johnson and A. Wightman. We expect the Institute to grow over the next several years as the Department of Physics plans to hire new faculty in the area of condensed matter theory. 


\section{PARTICLE THEORY PERSONNEL}

\section{(a) Faculty}

The following is a list of the faculty members of the particle theory group, their status and the length of their association with the group. Dallas Kennedy is a new member of the group. Dallas received his Ph.D. from Stanford University under Bryan Lynn and was a Research Associate at Fermilab before joining the group.

Name

T. Curtright

R. D. Field

D. Kennedy

Z. Qiu

P. Ramond

P. Sikivie

C. Thorn

R. Woodard

\section{Particle Theory Faculty}

\section{Position}

DOE Outstanding Junior Investigator

$(9 / 1 / 82-8 / 1 / 84)$

Assistant Professor

(9/1/84-9/1/85)

Associate Professor

$(9 / 1 / 85-8 / 31 / 89)$

Professor

(9/1/80-present)

Assistant Professor

(9/1/93-present)

Assistant Professor

(9/1/89-present)

Professor

(9/1/80-present)

Assistant Professor

(9/1/81-9/1/84)

Associate Professor

(91/85-8/31/88)

Professor

(9/1/88-present)

Professor

(9/1/80-present)

Assistant Professor.

(9/1/89-present)

\section{(b) Postdoctoral Fellows and Long Term Visitors}

In 1982, our first two postdocs were E. Braaten, now just recently tenured at Northwestern, and M. Chase who went on to CERN and then into industry in the UK. From 1983 to 1985 , we were joined by Richard Holman who went to Fermilab and is now an Assistant professor at Carnegie-Mellon. Our second crop of postdocs included M. Doria 
who went on to Los Alamos and is now in Brazil in a research position; J. Mc Cabe who was a postdoc from 1984 to 1986 and went on to LAPP in Annecy; and V. Rodgers who was with us from 1985 to 1987 and then went on to Stony Brook and is now an Assistant Professor at Iowa State.

In 1986, D. Harari joined our group. He is now in Buenos Aires in an academic position. Phil Oh from Chicago was here in 1986 and left having spent a year with us. He is now in a teaching position in Korea. In 1987, we were joined by D. Zoller, J. Minahan, S. Yost and A. Polychronakos, who was the first recipient of the IFT fellowship allocated to our group. In 1988, we acquired Christian Preitschopf from SLAC. In 1989, M. Awada came on board.

Name
E. Braaten
M. Chase
M. Sato
F. del Aguila
M. Doria
R. Holman
V. Rodgers
J. McCabe
P. Oh
D. Harari
J. Minahan
D. Zoller
S. Yost
A. Polychronakos
C. Preitschopf
M. Awada
S. Martin
S. Rey
S. Sin
Y. Wang
M. McGuigan
P. Griffin
K. Anagnostopoulos
M. Booth

Name

E. Braaten

M. Chase

M. Sato

F. del Aguila

M. Doria

R. Holman

V. Rodgers

J. McCabe

D. Zoller

S. Yost

A. Polychronakos

C. Preitschopf

M. Awada

S. Rey

S. Sin

Y. Wang

M. McGuigan

P. Griffin

K. Anagnostopoulos

M. Booth

Particle Theory Postdoctorai Fellows

Length of Stay

$(9 / 1 / 81-8 / 31 / 83)$

$(9 / 1 / 81-8 / 31 / 83)$

$(9 / 1 / 82-5 / 1 / 83)$

$(9 / 1 / 82-8 / 31 / 84)$

$(9 / 1 / 83-8 / 30 / 85)$

$(7 / 1 / 83-6 / 30 / 85)$

$(9 / 1 / 85-8 / 30 / 87)$

$(9 / 1 / 85-8 / 30 / 87)$

$(9 / 1 / 86-7 / 31 / 87)$

$(9 / 1 / 86-8 / 31 / 89)$

$(9 / 1 / 87-8 / 31 / 90)$

$(9 / 1 / 87-8 / 31 / 90)$

$(9 / 1 / 87-8 / 31 / 91)$

(IFT, $9 / 1 / 87-8 / 31 / 90)$

$(9 / 1 / 88-9 / 30 / 91)$

$(10 / 1 / 89-8 / 31 / 91$

$(9 / 1 / 90-8 / 31 / 92)$

$(9 / 1 / 90-7 / 31 / 91)$

(IFT, $9 / 1 / 90-8 / 15 / 92)$

(9/1/91-8/1/93)

(9/1/91-present)

(10/20/91-8/1/93)

(8/1/93-present)

(8/1/93-present)

(8/1/93-present)
Position after UF

Northwestern University CERN

Japan

University of Granada

Los Alamos

Fermilab

Stony Brook

LAPP

Korea

Buenos Aires, Argentina

University of Virginia

University of Cincinnati

University of Tennessee

Columbia University

Göteburg, Sweden

University of Cincinnati

Northeastern University

Korea

Han-Yang University

Fermilab

University of Florida

University of Florida

SSC Fellow at UF

University of Florida

University of Florida

In the summer of 1990, J. Minahan left to another postdoc at UVA and A. Polychronakos went to Columbia University. The extraordinarily competitive job situation for non-Russian non-phenomenologists that year resulted in neither Yost nor Zoller being able to secure a postdoc position at another institution. We chose to keep Yost for another 
In 1991 G. Kleppe received his Ph.D. in under Ramond's supervision and went on to a first postdoc position at V.P.I. and in 1992 another student of Ramond, Brian Wright, graduated and took a first postdoc position at the University of Wisconsin, Madison.

This year we are graduating five Ph.D. students. E. Piard, B. Keszthelyi, H. Arason, and D. Castaño were supervised by Ramond and S. Carbon is a student of Thorn. E. Piard and D. Castaño have accepted postdoctoral positions at the University of Virginia and M.I.T., respectively. H. Arason will teach in Iceland and S. Carbon has chosen to go into industry.

Name
M. Ruiz-Altaba
D. Hong
R. Viswanathan
M. Chu
C. Hagmann
T. McCarty
G. Kleppe
B. Wright
E. Piard
B. Keszthelyi
H. Arason
D. Castaño
S. Carbon
J. Kim
S. Mikaelian
J. Rubio
M. Tayebnejad
M. Kim
Y. Kanev
N. Irges
W.-L. Liu

\section{Particle Theory Graduate Students}

Advisor Position after UF

Ramond Ph.D. 9/1/87, University of Geneve

Ramond Ph.D. 9/1/88, Korea

Ramond Ph.D. 9/1/89, ICTP in Trieste, Italy

Thorn Ph.D. $4 / 1 / 90$, DAMPT in Cambridge, U.K.

Sikivie Ph.D. $8 / 3 / 90$, Berkeley

Ramond Ph.D. $8 / 3 / 90$, industry

Ramond Ph.D. $8 / 91$, V.P.I.

Ramond Ph.D. 8/92, University of Wisconsin

Ramond Ph.D. 8/93, University of Virginia

Ramond Ph.D. 8/93

Ramond Ph.D. 8/93, teaching in Iceland

Ramond Ph.D. 8/93, M.I.T.

Thorn Ph.D. 8/93, industry

Sikivie Ph.D. expected in 1994

Thorn Ph.D. expected in 1994

Woodard Ph.D. expected in 1994

Field Ph.D. expected in 1994

Sikivie Beginning research

Sikivie Beginning research

Ramond Beginning research

Qui Beginning research

Under our present funding profile, graduate students are supported by the department with teaching assistantships (TA) during the Academic Year. We support the students with DOE and IFT (and sometimes DSR) funds during the summer. In addition, whenever funds permit we would like to support students during the last stages of their Ph.D. research. 


\section{Scientific Statements of the Group Members}

\section{(a) R. D. Field}

Much of my current research is related to Hadron Colliders and the SSC. I would like to maintain a small but active group of students and postdocs working on Collider Phenomenology. At present I have one student, M. Tayevnejad ("Reza"), and one postdoc Paul Griffin working with me. I would like to take on several more students to work on phenomemology. I have spent a lot of time over the last year conducting an individual study program. The program consisted of two phenomenology lectures per week by myself together with assigned problems. Three entering graduate students (and three undergraduates) participated enthusiastically and I believe that my efforts will eventually result in more phenomenology $\mathrm{Ph} . \mathrm{D}$. students. Several of my students will be taking the Graduate Comprehensive Exam this fall and if they pass they will be able to start their research. Also, the recent hiring of Dallas Kennedy as a new Assistant Professor in our group should prove beneficial to Florida phenomenology.

Paul Griffin and I recently completed our first paper which is entitled "Enhancing the Heavy Higgs Signal with Jet-Jet Profile Cuts". The paper will appear in Physical Review $D$. The great challenge at hadron colliders is to disentangle any new physics that may be present from the "ordinary" QCD background. An important final state consists of a large transverse momentum charged lepton pair plus two accompanying jets $\left(i . e ., \ell^{+} \ell^{-} j j\right)$. It is one of the relevant signals for the production of a Higgs particle and its subsequent decay into $Z Z$ with one $Z$ decaying leptonically and the other $Z$ decaying hadronically into a $q \bar{q}$ pair which then manifests itself as a pair of jets. Unfortunately, too often the large transverse momentum production of single $Z$ bosons plus the associated jets mimic the Higgs signal. Once one requires the $Z$ boson to have a large transverse momentum by demanding a large $P_{T}$ lepton pair, one has forced the background to have a large $P_{T}$ "away-side" quark or gluon via subprocesses like $q g \rightarrow Z q$ or $q \bar{q} \rightarrow Z g$. This away-side parton often fragments via gluon bremsstrahlung, producing away-side jet pairs which resemble the signal. However, the signal jet pair and the background jet pair have quite different origins. The former arises from the decay of a color singlet $Z$ boson while the later is produced in a color non-singlet "parton shower". We examine in detail the jet-jet profile, or precise manner, in which transverse energy and mass are distributed around this jet-jet system.

Our method can help to distinguish the two jet system originating from $q \bar{q}$ the decay of a color singlet $Z$ boson from a random jet pair coming from the "ordinary" QCD gluon bremsstrahlung of colored quarks and gluons. The procedure can be summarized by the following series of selections and cuts:

- Lepton pair trigger.

- Jet pair selection.

- Jet-jet profile cuts.

- Jet-jet invariant mass cuts.

The profile analysis of the jet-jet system is accomplished by defining three "bi-polar" regions. The first region is the jet-jet core, corresponding to cells whose centers lie within 
a "distance" $R$ (core) in $\eta$ - $\phi$ space of either jet. The jet-jet center region corresponding to cells whose centers lie within $R$ (center) of either jet and the full jet-jet pair region is all the cells whose centers lie within $R$ (halo) of either jet. The jet-jet halo region corresponds to cells whose centers lie between $R$ (center) and $R$ (halo) of either jet. These regions are used to define observables that can differentiate between the jet pairs that originate from the hadronic decay of a $Z$ boson in the decay of the Higgs signal and the jet pairs that result from gluon bremsstrahlung from the recoil parion in large $P_{T}$ single $Z$ production (i.e., the background). The first observable is the fraction of the full jet pair transverse energy that occurs in the jet-jet halo region:

$$
F_{E_{T}}=E_{T}(\text { jet-jet halo }) / E_{T}(\text { full jet-jet }) \text {. }
$$

The second observable measured the invariant mass shift from the jet-jet cores to the full jet pair:

$$
\Delta M=M(\text { full jet-jet })-M(\text { jet-jet cores }) .
$$

These two quantities measure how transverse energy and mass, respectively, are deposited around the jet-jet cores. By making cuts on these observables, signal to background enhancement factors greater than 100 can be obtained. With enhancements this large, an $800 \mathrm{GeV}$ Higgs boson stands out in the invariant mass plot as a definite peak over the background.

In February, Paul Griffin applied for an "SSC Fellowship" based on our work and we sent a draft of our first paper with his application. I am happy to say that Paul was awarded the Fellowship, which provides one year salary with funds for travel and equipment. Paul plans to visit the SSC, but will spend most of the year here in Florida working with me. Also, we have guaranteed Paul an additional year after the SSC fellowship as a Postdoc with our group.

Paul and I are in the process of completing our second paper which extends our jet-jet profile analysis to include the $W W$ decay modes of the Higgs as well as the $Z Z$ channel. Our profile analysis works equally well on the away-side $W \rightarrow q \bar{q}$ system. However, for the $W W$ channel one has the $g g \rightarrow t \bar{t}$ background to contend with. We also discuss in detail the effects of experimental resolution on the profile cuts.

My graduate student, Reza, and I have been working on observing the Higgs in the purely leptonic channels (i.e., $l^{+} l^{-} l^{+} l^{-}$and $l^{+} l^{-} \nu \bar{\nu}$ ). We are examining ways of observing the $\mu^{+} \mu^{-} \tau^{+} \tau^{-}$and $e^{+} e^{-} \tau^{+} \tau^{-}$decay modes of the Higgs at the SSC. I am hoping that this work will produce a paper within the next year and that Reza can use it as part of his thesis.

I am a member of the Solenoidal Detector Collaboration (SDC) at the SSC and I have contributed to the collaboration by writing three SDC reports. In my first SDC report, I examined "jet shapes" in the forward region in high-energy proton-proton collisions at the SSC. Jets in the forward region (i.e., $\eta$ (jet) $>3$ ) arise from 2-to-2 subprocesses in which the parton-parton center of mass is "boosted" along the incident proton direction - Because of the Lorentz contraction "jets" no longer appear, on the average, as cones. The shape in the azimuthal angle $\phi$ remains roughly invariant, while the shape in $\theta_{c m}$ is 
compressed. Shapes in pseudorapidity remain roughly invariant except for slow particles where mass effects become important. This means that "jets" in the forward region look, in the laboratory, more like sections of a "disk".

In the second SDC report, I examined how much these forward jets contribute to the missing transverse energy if they are not measured (due to lack of rapidity coverage) or if they are inaccurately measured (due to, for example, a large calorimeter cell size and hence bad spatial resolution). Missing a forward jet (or assigning it the wrong transverse momentum) results in "fake" missing transverse energy (i.e., missing transverse energy that is not due to neutrinos, photinos, etc. ). "Fake" missing $E_{T}$ is a background to the true missing $E_{T}$ that can be used as a signal for new physics. Rapidity coverage turns out to be the key factor in reducing the "fake" missing transverse energy produced by ordinary QCD events. If one has incomplete rapidity coverage than occasionally one misses a forward (or backward) going jet whicn then is interpreted as missing transverse energy. In $40 \mathrm{TeV}$ proton-proton collisions this jet, when boosted to the forward direction, becomes a "disk" with an angular width of $\Delta \theta_{c m}<1^{\circ}$. Such a compressed jet can easily "squeeze" outside the rapidity coverage (carrying its transverse energy with it). In reducing "fake" missing transverse energy, the calorimeter cell size is not nearly as important as the rapidity coverage. Energy resolution is even less important than the cell size. From the "fake" missing $E_{T}$ point of view a crude forward calorimeter covering a large rapidity region is better than a good calorimeter covering a small rapidity range.

My third SDC report was written in collaboration with Paul Griffin. We extended our jet-jet profile analysis to include $600 \mathrm{GeV}$ Higgs bosons as well as $800 \mathrm{GeV}$ at the SSC.

I am not necessarily a proponent for heavy Higgs bosons. I believe that we should be prepared for anything and the jet-jet profile analysis works particularly well for high mass Higgs. In particular, the jet-jet profile methods works well if the transverse momentum of the away-side $Z \rightarrow q \bar{q}$ system is greater than about $200 \mathrm{GeV}$. For lower $P_{T}$ values the signal profile begins to resemble the background making the cuts less effective. We are investigating other techniques for lighter Higgs boson masses. If there is no Higgs particle, but instead the Weak interactions become strong at around $1 \mathrm{TeV}$, then the jetjet profile techniques should help in observing the strongly interacting subprocesses such as $W W \rightarrow Z Z$ and $Z Z \rightarrow Z Z$. We plan to look at this more closely in the future.

My overall goal over the next several years is not the Higgs boson, but supersymmetry. Using the tools that we have developed by examining the Higgs boson, I plan to conduct a detailed study of supersymmetry at the SSC. In particular, I would like to learn how to disentangle various supersymmetric signals from the ordinary QCD background at the SSC.

\section{(b) D. Kennedy}

My current research is concentrated in two areas. The first is the theory and phenomenology of electroweak gauge interactions. A major part of this lies in the area of electroweak radiative corrections, starting with my thesis work at Stanford (with Bryan W. Lynn), which ended up in a complete reformulation of the subject expressed in the so-called "star" system of momentum-dependent couplings and embodied in a radiative 
corrections Monte Carlo program (EXPOSTAR) for electron-positron colliders; gaugeinvariant classificatory methods for corrections, since developed further by other workers and applied to various kinds of electroweak processes; and a general treatment of heavy particle effects in electroweak corrections, which formed the starting point for the so-called " $S, T, U$ " analysis of electroweak data. With Paul Langacker at Penn, I have participated in this analysis myself in the last three years, deriving from precision electroweak measurements (such as the LEP data) tests of the Standard Model and constraints on non-Standard physics. The formal and phenomenological aspects were united in my 1991 TASI lectures on the subject. The subject deserves summarizing in the form of review articles, of which I plan to write two: one on electroweak data analysis, the other on formal aspects of radiative corrections in general non-Abelian gauge theories. I also plan to correct and revise my Monte Carlo code into a more general and easier-to-use form appropriate for arbitrary colliders, with the help of a computer science student.

Recently, I have worked with Chris Hill at Fermilab on a new approach to dynamical electroweak symmetry breaking (technicolor), based on breaking the technicolor group itself. Having finished a theoretical paper on this subject, I am planning a phenomenological paper or two, to outline what such theories would imply for very energy collider physics and astrophysics.

The second major area of my research has been in neutrino physics, particularly the solar neutrino problem. I began this work at Penn (Bludman, Hata, Langacker) by giving the 1990 TASI lectures on the subject. My collaborators at Penn and I have completed two major papers on solar neutrino data, based on a code partially written by myself that analyzes the data in terms of the Mikheyev-Smirnov-Wolfenstein resonant flavor oscillation effect and a simplified treatment of solar model variations. I have also done some work on neutrino mass models, which I would like to extend further into supersymmetry, lepton number violation, grand unification, astrophysics, and cosmology. The Penn collaboration will extend our work to analysis of new data from present experiments (Homestake, Kamiokande, SAGE, and GALLEX) and planning for future experiments, in particular, SuperKamiokande, Borexino, and the Sudbury Neutrino Observatory. I also hope to continue working on a more speculative idea, a systematic and more accurate method for treating models of the Sun and variations thereof, in order to separate solar from particle physics effects in the solar neutrino problem.

In the past, I have done some work on non-perturbative field theory (solitons, strong interactions), which may provide a source of future research ideas. I continue to retain a strong, if outsider, interest in the dynamics of nonlinear and complex systems (generalized statistical mechanics).

Present Research Plans:

- Electroweak reviews: (1) data analysis and (2) formal issues.

- New version of electroweak Monte Carlo EXPOSTAR.

- One or more papers on broken technicolor phenomenology.

- Solar neutrino data analysis of present and future detectors.

- Formal structure of solar models, as related to neutrinos. 
- Models of neutrino mass and mixing.

\section{(c) Z. Qiu}

It is well known that the bosonic string theory is not a consistent theory due to the existence of a tachyon. One of the goals of formulating string field theory is to discover the true vacuum of string theory. Work in this direction so far yields few insights due to our lack of understanding of closed string field theory. Moreover if the true bosonic string vacuum turns out to be a string theory, we should be able to understand it before understanding the string field theory. The question of whether there is a consistent $d>1$ bosonic string theory is a fundamental one and deserves further exploration.

In a recent paper, I consider a string theory with two types of strings with geometric interaction. I show that the theory contains strings with constant Dirichlet boundary condition and those strings are glued together by 2-d topological gravity with macroscopic boundaries. A light-cone string field theory is given and the theory has interactions to all orders. This is a string theory that incorporates non-critical $d \leq 1$ strings into critical bosonic string theory. In the first quantized language, the amplitude of the string theory has new contributions from "colored" Riemann surfaces, black and white in our case, which come from interactions between two types of strings. The white region represents ordinary bosonic string with suitable boundary condition and the black region the noncritical strings. Therefore in the calculation of amplitudes in the theory one not only has to sum over all surfaces but also has to sum over the coloration and all possible black strings as well.

There are many interesting questions one can consider following this idea. How to determine the exact mass spectrum of the modified bosonic string theory? The covariant formulation of the theory is another interesting problem in its own right.

The extension of these results to superstring is of considerable interest. Instead of topological gravity, we should consider topological supergravity as the topological sector of superstring. By including the topological sector and interacting sector to superstring, it is conceivable that the theory automatically gives an expectation value to dilaton and breaks supersymmetry. The reason is that the point-like structure from the topological string couples only with scalar fields of superstring.

I also study the role of interacting sector in string theory with $d>1$. In a recent paper, UFIFT-93-8, I quantize the theory with point-like interaction in conformal gauge. I consider the non-critical string theory in dimensions $1<d<25$ and study the scaling behavior of the partition function. The "string susceptibility" is calculated. The comparison with $d \leq 1$ non-critical string theory is made and the interpretation of the so-called " $c=1$ barrier" is addressed. I also consider the quantization of the theory in critical dimensions in conformal gauge.

The main result of the paper is that I give a procedure to find the new fixed point of non-critical string theory of $d>1$. The scaling properties of the new string theory is discussed. The first step is an observation that there is a trivial string theory for every dimensions consisting of a collection of string theories with point-like target space, 
e.g. 2-d topological gravity for each space point, with some density to be determined. It is not a very interesting string theory because the theory has trivial space dynamics. The next step is to turn on space fluctuations of the surface. By coupling of point-like strings of the trivial string theory with fluctuating surfaces in space with geometric string interaction. I introduce a parameter $\hat{\epsilon}$, "chemical potential" which controls the ratio of the contributions from point-like strings and that from fluctuating surfaces. Varying of $\hat{\epsilon}$ enables us to interpolate to a new "fixed point" with fluctuating surfaces dominating the path integral. It is this new "fixed point" that gives a non-trivial $d$ dimensional noncritical string theory. A new scaling relation is derived and its solution gives the "string susceptibility" of the new fixed point.

It is obvious that the above prescription hints a much simple formulation of the problem in terms of matrix model. Works in this direction are in progress. My work also provides a "stringy" way to deferm one non-critical string theory to other. It could help us to understand better the space of all string theories.

There are close connections between the study of string theory and that of two dimensional conformal field theory. The two-dimensional conformal field theory is a very powerful tool in studying the properties of physical systems where the relevant degrees of freedom exhibit local scale invariance. The familiar examples are the two-dimensional critical phenomena and the properties of strong coupling fixed point of Kondo impurity system.

In fact, many recently advances in these two subjects are based on the advance in the mathematical structure shared. The more familiar examples are the connections of closed string theory and the bulk conformal field theory, open string theory and boundary critical phenomena, non-ghost theorem in string theory and the question of unitarity in conformal field theory, superstring and superconformal field theory, modular invariance as consistent condition of string theory and its role to determine the spectrum of conformal field theory.

I consider the interferences in conformal field theory. In particular, two conformal field theories sharing a common boundary. The central object of interesting is a "interacting vertex" connecting two Hilbert spaces. The study of conformal interference also provides the mathematical tool needed in studying interactions of different types of strings.

\section{(d) P. Ramond}

\section{Recent Activities:}

In 1990, my students and I undertook a mammoth project on plotting all of the Standard Model's parameters up to the Planck scale. This work includes proper account of thresholds, two loop effects, and an algorithm for bringing the initial data to the same scale. The work was published in the Physical Review. The idea is to use this work as a template upon which new models are added. For several of the students, this paper has been an important part of their thesis.

In spring 1991, we had to depart from our game plan which was first write the template, then add supersymmetry. The work of Amaldi et al. forced us to publish over that 
summer a paper on the Yukawa couplings in the minimal supersymmetric extension of the Standard Model. In it we use the SUSY unification scale to demand that the bottom and tau Yukawas be the same at that scale. Then, after comparison with upsilon physics at $10 \mathrm{GeV}$, we infer from the running of the couplings bounds on the top quark masses. Remarkably, we found that the top quark mass comes out less than $200 \mathrm{GeV}$, in accordance with the $\rho$ parameter bound. This was the first time that supersymmetry has been shown to improve GUT predictions in the Yukawa sector. This work appeared in Physical Review Letters in the summer of 91 .

We continued our work on the Yukawa to include the three families. We used as input the Yukawa matrices that Jeff Harvey, David Reiss, and I had formulated in 1980, based on the work of Georgi and Jarlskog. One of the predictions was a relation between the lifetime of the B meson and the top quark mass. We showed that with supersymmetry, this relation is not inconsistent with data, provided the top quark is almost as heavy at it can be. This work was published in the Physical Review.

I wrote a preliminary version of this work for the Festschrift volume of my colleague, John Klauder, to be published by World Scientific. I may note that this work is similar to that of Dimopoulos, Hall and Raby. Ours was done with students, and got much less publicity, but our results were no less significant.

My research efforts got somewhat slowed down when I accepted to serve on the HEPAP Whitherell subpanel early in 92. This essentially took me out of research for several months. I was delighted to find that my students were sufficiently along to carry the research ball by themselves. In fact, we have another big paper in preparation, the supersymmetric version of the template paper. As of this writing, extensive numerical work has been done: we now have a program where you input the soft SUSY breaking parameters at GUT scale, and out pop the values of the sparticle masses, the top quark mass, etc... The assumptions are just supergravity inspired flavor blind breaking, radiatively induced electroweak breaking. I presented the results for the no-scale models at the HARC conference in April 93, and produced a preprint to a ppear in the proceedings. The big paper which presents the numerical results for more general supersymmetry breakings is being finished as of this writing.

With Graham Ross (Oxford) and Dick Roberts (Rutherford), I have been working on a catalogue of Yukawa matrices that are consistent with present data. I presented preliminary results at the Coral Gables conference in January 93, to appear in the proceedings. The complete work was preprinted in April and has been accepted for publication in $\mathrm{Nu}$ clear Physics B. I shows that there are but a few textures consistent with present data. These textures carry predictions of their own which must await further experiments for verification.

Lately I have been a guest scientist at the SSCL where I have renewed a collaboration with a former postdoc at Florida, Steve Martin. We had started collaborating on this project last October at a Yale workshop, and we have just finished a paper which presents new sum rules among superparticles, based on the ideas of supergravity and softly broken supersymmetry. In this case the work is not numerical! We are submitting the paper to the Physical Review D. 
I have also presented public talks aimed at general audiences, one in Aspen in July 92, one at $U$ of $F$ in the Frontiers of Science series in the Fall of 92, one at the Berkeley School in Tampa in October 92, and the last one at the School for Future Scientists in June 93.

Lastly, as Director of IFT, I supervised the IFT's first workshop on Dark Matter; I am part editor, with the proceedings to be published by World Scientific.

\section{Future Directions:}

In the near future I have contracted with Addison-Wesley to write an advanced texbook in the Frontiers of Physics series; it is to be called "Journeys Beyond the Standard Model", and it is more than half-way done. This fall, I will teach the course on the standard model, which will enable me to finish the book.

In terms of research, I have spent some time on developing a Landau-Ginzburg theory in $2+1$ dimensions. With a postdoc here, McGuigan, I have been looking at vortex solutions. This is an attempt to generalize the work of Nagaosa and Lee, which is based on ideas of Larkin and Ioffe. Much of this was motivated by conversations with Bob Schrieffer who told me of ideas on gapless superconductivity. I noticed that in $2+1$ dimensions, there are two possible order parameters, that is operators with naive dimensions less than three; they are the usual fermion pair, and the two fermion-one boson operator. I have been intrigued by the possible role of this operator in theories of high $T_{C}$. I have not been working hard on this problem of late, but aim to devote my time to it in the fall.

I also plan to pursue the research of the last three years in exploring several variations of the soft supersymmetry breaking. Of particular interst is the role of the Peccei- Quinn symmetry, and also studying the overlap with string theories. This ties in with the study of Yukawa textures where the presence of simultaneous zeros in the Yukawas may indicate hints of geometrical structure in the coset manifold; for instance a zero may be interpreted as a lack of intersection of various curves in the manifold. I plan to look at these questions in the coming year, and also continue bringing up to steam $\mathrm{Mr}$. Irges.

\section{(e) P. Sikivie}

Last spring, Diego Harari and I wrote a paper on the effects of a massless NambuGoldstone boson on polarimetry in astronomical observations. We showed that the observed correlation between the directions of elongation and of polarization of distant radiogalaxies places an upper bound on the coupling strength of a Nambu-Goldstone boson $\phi$ to two photons. If we write this interaction as $L_{\phi \gamma \gamma}=g_{\phi \gamma \gamma}\left(\frac{\alpha}{\pi v}\right) \phi \vec{E} \cdot \vec{B}$ where $v$ is the magnitude of the vacuum expectation value that breaks the global symmetry responsible for the existence of $\phi$, and $\vec{E}$ and $\vec{B}$ are the electromagnetic fields, than $g_{\phi \gamma \gamma}<50$. This bound arises because the plane of polarization of a photon rotates while the photon travels in regions where the $\phi$ field is spatially and/or temporally varying and it is expected of the cosmological $\phi$ field that it changes by order $v$ over the horizon scale. We also showed that the observed lack of polarization of the cosmic microwave background places a lower limit on $v$ of order $10^{5} \mathrm{GeV}$. This bound arises because photons convert to $\phi$ Nambu-Goldstone bosons in astrophysical magnetic fields through the interaction $L_{\phi \gamma \gamma}$. 
Jaewan Kim, Jooyoo Hong and I wrote a paper in which we obtain the relativistic equations of motion for strings with arbitrary equation of state, i.e. with arbitrary relationship between tension $\tau$ and energy per unit length $\epsilon$. We also derive the renormalization of $\tau$ and $\epsilon$ due to small wiggles in the string, to lowest order in the wiggle amplitudes for general strings but to all orders for Nambu-Goto strings. We find deviations from the equation of state $\epsilon \tau=$ constant which had been proposed by previous authors for wiggly Nambu-Goto strings. We also irgue that wiggliness may dramatically alter the cosmic gauge string scenario.

Stephen Hsu, who is a Junior Fellow at Harvard, and I wrote a paper in which we compute the long range force due to two neutrino exchange. It is of order $G_{F}^{2} r^{-6}$, which is far too small to be observed in any foreseeable experiment. It turns out that, unbeknownst to us, this force had already been calculated by Feinberg and Sucher about 25 years ago. Our results agree with theirs except for a relatively minor point: a factor of three in the relative strength of one of the spin-dependent terms.

Eric d'Hoker, who is a faculty member at UCLA, and I wrote a paper on the Casimirlike force between two beads on a straight string. We calculate the quantum-mechanical zero point energy of the string as a function of the distance $r$ between the two masses. The resulting force between the beads is attractive and falls off as $\frac{1}{r^{3}}$ for large distances (or small masses) and as $\frac{1}{r}$ for short distances (or large masses). The attractive nature of the force creates an instability under which masses added to the string tend to aggregate.

David Tanner, Yun Wang and I wrote a paper describing an experimental scheme to search for galactic halo axions with mass $m_{a}$ in the $10^{-3}$ to $10^{-5} \mathrm{eV}$ range. The cavity technique becomes unwieldy when $m_{a}$ is larger than a few $10^{-5} \mathrm{eV}$. The detector we propose consists of a large number of parallel superconducting wires embedded in a material transparent to microwave radiation. The wires carry a current configuration which produces a static, inhomogeneous magnetic field wherein axions may convert to photons. We discuss the feasibility of the detector and its sensitivity.

Yun Wang, Igor Tkachev, who is a postdoc at Fermilab, and I are studying the radial infall model of galactic halo formation. This model assumes that galactic halos form by accreting dark matter devoid of angular momentum. This assumption is probably unrealistic. Nonetheless the model has had some success in producing flat or nearly flat rotation curves. We are particularly interested in the model because it makes a prediction for the phase space structure of galactic halos and hence for the peaks in the energy spectrum of cold dark matter particles on earth. These peaks would be readily seen in a cavity detector of galactic halo axions if such a detector were to find a signal. We have developed a number of numerical and analytical techniques to investigate the model and are beginning to obtain a consistent picture of it's behaviour and properties. We hope to finish our paper on this topic before Yun Wang leaves for Fermilab at the end of the summer.

Jaewan Kim and I are investigating the response of short wavelength wiggles on a string to uniform stretching of the string. The formalism we developed a year and a half ago, in collaboration with Jooyoo Hong, allows us to analyze this problem thoroughly. The 
results can be used to analyze the behaviour of cosmic gauge strings. We are presently writing a paper on this subject.

\section{(f) C. B. Thorn}

During 1990 I worked on "subcritical string theory", the theory of strings in less than the critical space-time dimension, developing an open string field theory for subcritical dimensions. These results should be useful in further understanding the matrix model approach for $D \leq 1$ as well as subcritical string theory for $1 \leq D \leq 25$. This and related work was published in a Nuclear Physics B article and two Physics Letters.

One issue raised by progress in subcritical (i.e., $D<26$ ) string theory is the fate of the graviton so prominent in the critical theory $(D=26)$. Unfortunately the only really complete treatment of subcritical string theory, via matrix models, works only for $D \leq 1$, where the issue of gravitons does not arise. Partial understanding for the case $1 \leq D \leq 25$, indicates the absence of a massless spin 2 particle in the spectrum. The massless spin 2 particle for $\mathrm{D}=26$ forces a gauge group at least as rich as general coordinate invariance. Normally we associate such a gauge group with massless gravitons, so what could be happening in $D<26$ ? In 1991, with Michael Green, we studied an example in string theory, viz. open string theory in tree approximation where there is no massless graviton. We constructed an interpolation between this theory and closed string theory (which has a graviton). We showed that the intermediate theories do have ghosts and clarified the manner in which consistency is regained in the two limits. This example, shows how the generic problems expected from giving the graviton a mass might conceivably disappear for a discrete value of the mass. This work was published in Nuclear Physics B.

Also in 1991, I worked on developing the idea that relativistic strings are composite objects. This is motivated by the desire to bring string theory into the framework of ordinary quantum field theory, and also by the growing feeling that the apparatus of string field theory is overly cumbersome and perhaps not even internally consistent. If strings were bound states of ordinary field excitations, it would be wrong to assign string states a fundamental role in the formulation of the dynamics. I reworked the so called "fishnet" model of composite strings into an explicit postulate for an ordinary (unfortunately not Lorentz covariant) field theory on a D-1 dimensional manifold. This exercise shows that it is possible to get the rich structure of string theory from even less than a field theory in $\mathrm{D}$ dimensions, but it is hardly the final answer, lacking Lorentz covariance for one thing. A field theory underlying strings, if it exists, should have general coordinate invariance, but neither strings nor a graviton as input. As candidates for such theories I plan to investigate "topological gravity" theories and "conformal supergravity" theories. These are both examples of generally covariant field theories without input gravitons, and as such might have some light to shed on string theory. These ideas were published in the proceedings as an invited talk to the Sakharov Conference in Moscow (May 1991), and I have continued attempts to develop them in 1992.

One of the driving motivations for thinking about subcritical string theory is the old hope that string theory might be a useful tool for describing quark confinement in QCD. While it is undoubtedly true that the 't Hooft's $1 / N_{c}$ expansion of QCD leads to 
some kind of a string theory (assuming quark confinement), this "QCD string" is quite a different object than the subcritical string obtained from dual models. For one thing, the QCD string must be compatible with asymptotic freedom and contain hard point like structures. For another, QCD is not generally covariant and hence could never produce a graviton in a consistent approximation. In 1992, M. McGuigan and I calculated the large $t$ behavior of quark-antiquark Regge trajectories in large $N_{c}$ QCD. Because we work at large $t$, perturbation theory is reliable. We published this work in Physical Review Letters. A striking implication of these results is the prediction that the $\rho$ trajectory should approach zero from above as $t \rightarrow-\infty$. Since existing measurements of the $\rho$ trajectory indicate that it crosses zero at $t \sim-.5 \mathrm{GeV}^{2}$ and decreases further to around -.7 for $t \sim-7 \mathrm{GeV}^{2}$, this raises questions about how the $Q C D$ predictions square with the real world. There is persistent evidence that the measured value of $\alpha_{\rho}(t)$ increases when it is extracted from higher energy data. In Fall 1992 a graduate student Mikaelian and I studied the trends of existing data in order to estimate when asymptopia should set in and to decide whether future experiments might be able to resolve the discrepancy. We fit the data to a superposition

$$
\mathcal{M}=\beta_{1}(t) s^{0}+\beta_{2}(t) s^{\alpha}(t)
$$

where the first term is a crude representation of the hard parton $Q C D$ prediction and the second term serves to parametrize the soft hadronic part of the process. We found that a value of $\beta_{2} / \beta_{1} \sim 20$ gives a rough acount of the energy dependence found in the measured $\rho$ trajectory. For extremely high $s$, say $4000 \mathrm{GeV}^{2}$ the $Q C D$ term should stand out clearly. I presented these results to a conference at Coral Gables in January 1993. Mikaelian is currently seeking more understanding of the apparent suppression of hard components in Regge scattering in the context of a simple potential model that mimics the scaling violations of $Q C D$.

$\mathrm{My}$ interest in finding more fundamental formulations of string theory continues. One direction I will pursue is a systematic study of the short distance structure of string theory via high momentum transfer scattering amplitudes. One such study which offers some insight into the physics at short distances involves an exploration of the role "sister regge trajectories" play at high momentum transfer. This study is a major component in the research of my student Carbon who received his $\mathrm{Ph}$. D. in May 1993. We eventually plan to publish this work in a joint paper.

I spent May and June 1993 attending a research program on strings and black holes at the ITP in Santa Barbara. There has been a lot of recent attention to the information loss problem associated with Hawking radiation from black holes. I learned to my surprise that there is no consensus among the experts whether the apparent loss of quantum coherence in black hole evaporation is an issue of Planck scale physics or one of much larger distance scales. In the former case, we can hope that alternatives to Einstein gravity, such as string theory, which only depart from Einstein gravity at the Planck scale could resolve the paradox. In the latter case, string theory can't help and quantum theory would require drastic modification. This issue of Planck scale versus larger scales should be resolvable with our current understanding of gravity and I plan to spend time in the next few months getting to the bottom of it. 
In the next few years I expect my research efforts to involve:

1. Continued efforts to find a more fundamental formulation of string theory. Progress here would of course have important implications for quantum gravity and perhaps even the black hole information problem (if that is indeed a Planck scale issue).

2. Consideration of possibilities for using existing accelerators (e.g., Tevatron) to get a better handle on Regge trajectories in the regime predictable by perturbative $Q C D$. In this connection Brodsky, Tang and I have written a recent paper which provides reliable estimates for the "hard QCD" part of the Regge amplitudes. These estimates confirm that higher energies are needed to test the QCD predictions. There is much more work to do in assessing whether these predictions can be tested in collider experiments. (So far only fixed target experiments have been used to extract Regge trajectories.)

3. Continued efforts toward gleaning more theoretical information about QCD. I am still pursuing a bootstrap approach to solving large $N_{c}$ QCD. In addition, I have become involved in thinking about how more information can be obtained from Monte Carlo lattice gauge theory simulations.

\section{(g) R. Woodard}

My basic interest is quantum gravity in the larger context of Lagrangian field theory and particle physics. There are a host of interpretational issues in this field as well as three major phenomenological problems. The long distance - or "infrared" - problem is that Einstein's theory has an apparently free parameter called the "cosmological constant" whose value must be fine tuned to one part in $10^{120}$ in order to prevent the radius of the universe from either shrinking to near zero or else expanding exponentially. The short distance - or "ultraviolet" - problem is that quantum corrections to Einstein's theory diverge in an uncontrollable way. There is also a semi-classical problem having to do with claims for a breakdown of quantum mechanics in the evaporation of black holes.

I have spent most of the past two years studying the infrared problem in collaboration with Professor Nicholas Tsamis of the University of Crete. We first resolved a longstanding paradox to explain why all previous formulations of perturbative quantum gravity on de Sitter space fail to reproduce even the known classical limit. We then solved the linearized theory in toto and produced the first correct set of Feynman rules. The associated papers are HEP-92-20 - which appeared as Phys. Lett. B292 (1992) 269 - and HEP-92-14 - which has been approved for publication in Communications in Mathematical Physics.

Although the Hilbert action does not give a fully consistent quantum theory on account of the ultraviolet problem it can still be used reliably in the far infrared. We have recently exploited this fact, and new our formalism, to propose an explanation for why the currently observed cosmolomical constant is so small. This work is discussed in HEP-92-23 - which appeared as Phys. Lett. B301 (1993) 483 - and HEP-92-24. Our proposal is that the real cosmological constant is on the GUT scale. In the very early universe its effect would still have been negligible compared with high initial temperature. As the universe 
expanded it cooled, until finally thermal effects ceased to dominate the stress tensor. At this point inflation began; it also became possible to sustain the long-range correlations necessary to mediate infrared effects. As time progressed these infrared effects gradually slowed the rate at which the background geometry expanded. Any such slowing would manifest itself as a decay of the effective cosmological constant. A crude estimate of the lifetime is consistent with enough inflation to explain the homogeneity and isotropy of the observed universe.

Strong infrared effects arise because gravitons possess two properties that make them unique among the known massless particles: first, they self-interact through couplings of dimension three; second, their dynamics is not conformally invariant. The latter property enables gravitons to experience the redshift of a conformally flat, expanding universe. This redshift populates the low energy sector more fully than in flat space and so enormously enhances its importance. Because no other known massless particle can experience the gravitational redshift soft gravitons must dominate the physics of late times in an inflating universe, no matter how small the gravitational coupling constants are with respect to the matter ones.

The reason the expansion rate slows is that infrared loop effects give the graviton a negative self-en rrgy. This engenders a negative vacuum energy which is of course a source for the expansion of the background geometry. The reason this should drive the effective cosmological constant all the way to zero is that the graviton self-energy is not just negative but also infrared divergent if one assumes exponential expansion at late times, no matter how small the asymptotic time constant.

Current work concerns the limitations of our perturbative analysis. Because the most interesting density perturbations would have originated in the initial stages of inflation where perturbation theory is still valid, we are able to make a reliable estimate for $\delta \rho / \rho$. This work is being done with Professor Robert Brandenberger of Brown University. We are also working on a scheme in which all but the lowest modes of gravity are truncated and the resulting quantum mechanical system is solved numerically. Finally, we are trying to extend the analysis to the case of negative cosmological constant.

I have also produced two papers on interpretational issues and am working on a third. The first, HEP-92-16 - which appeared as Class. Quantum Grav. 10 (1993) 483 - proposes an inner product that can be used even when the spatial manifold is closed. The second, HET-92-18 - which has been approved for publication in Journal of Statistical Physics - was written in collaboration with Professor Peter T. Landsberg of the University of Southampton. We propose a technique through which ersatz ensembles can be used to study systems with negative heat capacities. (Because black holes and stars have negative heat capacities they cannot exist in equilibrium with thermal reservoirs.) The third paper is a collaboration with my graduate student, Jose Rubio. It concerns a way of defining the energy for constrained systems in which $H=0$ is one of the constraints. We applied our method to the famous problem of gravity on the manifold $T^{3} \times R$. The result is an energy functional which is positive semi-definite for perturbations about flat space. This functional also agrees with the ADM energy in the limit of infinite volume and localized, asymptotically flat initial value data. 
I have not worked on the ultraviolet problem during the past year although a long-term goal is to check the ansatz Dr. Gary Kleppe and I made for the measure factor in non-local regularization. Once this is proved our method can be used to generate new fundamental models of any non-anomalous theory, including gravity. The scale of non-localization would represent a new fundamental constant. Dr. Kleppe and I recently discovered a simple functional proof that our method provides a regularization of massless QED that gives simultaneously conserved vector and axial vector currents, without fermion doubling and without a phenomenological problem with $\pi^{0} \longrightarrow \gamma \gamma$. (The key to understanding the last point is that its nonlocality allows our axial vector current operator to interpolate for the state $\left|\pi^{0} \gamma \gamma\right\rangle$ in addition to just $\left|\pi^{0}\right\rangle$.) We are planning to write this up during the summer.

Although I have more than enough to work on with these other projects I have not been able to stop myself from thinking about the issue of black hole evaporation. It seems to me that a portion of the alleged paradox arises from illegitimately conceiving of the black hole as a classical source. Suppose that a tiny bit of matter falls into a black hole. Since it is provably true that the transient effects decay exponentially, we are told that most correlations with the outside are lost. Since it is also provably true that the extra mass added by the matter increases the source of Hawking radiation we are told that almost all of the stress-energy and charge of the bit of matter must come off in the form of thermal radiation. I do not accept this. Hawking radiation arises most strongly in the immediate vicinity of the event horizon where the causal structure of spacetime is quite peculiar. In fact since the event horizon is a null surface, and since even massive propagators are singular on the light cone, it seems to me that radiation from the black hole can be very strongly correlated with the in-falling bit of matter. Once this is conceded for a small extra bit of matter it follows as well for the original mass whose collapse produced the black hole. I shall probably write up something along these lines this summer during the workshop on the small scale structure of spacetime at the Institute for Theoretical Physics in Santa Barbara.

\section{(h) P. Griffin}

During the past six months, (October 1992 to the present), I have studied nonperturbative methods in light-cone field theory and Superconducting Supercollider (SSC) phenomenology via Monte-Carlo techniques.

I proved a non-renormalization theorem for contact terms in my formulation of staggered fermions on the transverse lattice[1]. The transverse lattice regularization of four dimensional field theories employs a two-dimensional space-like lattice to regulate the usual transverse ultraviolet divergences in light-cone field theory. This formulation is one of the most promising methods for studying non-perturbative four-dimensional gauge theories in the context of light-cone quantization. The primary goal of the light-cone method is to provide the detailed picture of hadrons, (structure functions, etc), that is difficult to obtain from lattice Monte-Carlo techniques. The theorem I proved shows that a certain class of counter-terms is not required in the lattice action to achieve the continuum limit. 
I also worked with Professor R. Field on Monte Carlo analysis Higgs boson events at the SSC. We developed a new method of differentiating the decay of a Higgs boson into two $\mathrm{Z}$ bosons, one of which subsequently decays into charged leptons and one of which decays into two jets, from the primary background. Our method is based on the difference in the color structure of the signal and background events. We used realistic Monte-Carlo studies of both signal and background to verify that our method is a viable one. If the Monte-Carlo analysis is taken as a good indication of what really will occur at the SSC should the Higgs boson really exist, then our method increases the signal by roughly a factor of two for a fixed amount of background, compared to previous analysis for this important discovery mode of a heavy Higgs.

We are preparing further analysis of heavy Higgs production and differentiation from background via our method for other decay channels of the Higgs, and for other possible Higgs masses (we considered a Higgs boscn of mass $800 \mathrm{GeV}$ in our original work). We also intend to study the experimental signature of Supersymmetry at the SSC via Mote-Carlo techniques in the coming year.

Because of our progress in this field, I have been awarded an SSC fellowship from the Texas National Research Laboratory Commission for the coming year. In addition to supporting me for one year, the fellowship money will enable us to increase our statistics on the difficult to study backgrounds via the purchase of faster computer workstations.

[1] Staggered fermions and chiral symmetry breaking in transverse lattice regulated QED, by P. A. Griffin, Physical Review D47 (1992)3530-3542.

[2] Enhancing the heavy Higgs signal with jet-jet profile cuts, by R. D. Field and P. A. Griffin, to appear in Physical Review D.

\section{(i) M. McGuigan}

A paper entitled "Charged black holes in two dimensional string theory" (Nucl. Phys. B375, 421 (1992)) was completed in collaboration with S. Yost and C. Nappi. This paper discussed various solutions to two dimensional dilaton gravity coupled to gauge fields, and derived a two dimensional heterotic string theory with charged black hole solutions.

A paper entitled "Quark-Antiquark Regge trajectories in large N QCD" (Phys. Rev. Lett. 69, 1312 (1992)) was completed in collaboration with C. Thorn. See the summary by him for a discussion of this work.

In addition the following projects are near completion: 1) "Third quantization and black holes" discusses the quantization of two dimensional dilaton gravity and its implicetions for black hole physics. 2) "CPT violation in antihydrogen and kaon physics" discusses the effect of small violations of quantum mechanics or loentz invariance on measurements in these systems. 3) "Single quark transitions in $K \rightarrow \pi \pi \gamma$ " discusses the effect of a large matrix element of a single quark decay in this process. 


\section{ACTIVITIES OF THE PARTICLE THEORY GROUP (since 1991)}

\section{A. Lect ures and Seminars}

Field

Talk presented at the SDC Collaboration Meeting, Argonne National Laboratory, March 20-23, 1991

High Energy Physics Seminar presented at the Institute for Fundamental Theory, University of Florida, Nov. 15, 1991

URA Fermilab Review, Jan 17-18, 1992

Physics Colloquium presented at the University of Florida, Feb. 6, 1992

High Energy Physics Seminar presented at the Institute for Fundamental Theory, University of Florida, Nov. 6, 1992

URA Fermilab Review, February 5-6, 1993

Seminar, SSCL, Dallas, TX, June 7, 1993

Qiu

Theoretical Physics Seminar, Cornell University, August 1992

Seminar, University of Florida, September 1992

Colloquium, University of Florida, October 8, 1992

Seminar, University of Florida, January 1993

\section{Ramond}

Seminar, Johns Hopkins, May 1991

SSC Colloquium, Dallas, Texas, May 1991

Invited Participant XXth Conference in Differential Geometry in Physics, New York, June 1991

Invited Lecturer, TASI Summer School, Boulder, Colorado, June 1991

Invited Lecturer, Johns Hopkins Workshop, August 1991

Seminar, University of Virginia, September 1991

Invited Lecturer on the occasion of ECG Sudarshan's 60th birthday, Austin, Texas, September 1991

Colloquium, U of Delaware, Nov 1991

Colloquium, Johns Hopkins, Nov 1991

Seminar, Princeton University, May 1992

Colloquium, SSC, June 1992 
Public Lecture, Aspen, Co, July 1992

Invited Lecturer, Erice, Italy, Sept 1992. Title: Neutrinos in Particle Physics

Seminar, Ecole Normale Supérieure, Paris, Sept 1992

Invited Lecturer, Yale Workshop, Oct 1992

Invited Lecturer, Coral Gables Conference, Miami, Jan. 1993

Invited Lecturer, HARC, Houston, TX, April 1993

Seminar, Johns Hopkins University, Baltimore, MD, April 1993

Colloquium, University of Chicago, May 1993

Seminar, Southern Methodist University, Dallas, TX, May 1993

Colloquium, SSCL, Dallas, TX, June 1993

\section{$\underline{\text { Sikivie }}$}

Lecture at the First International School on Particle Physics and Cosmology, in Baksan Valley (Caucasus), USSR, in May 1991

Seminar at the Institute for Nuclear Research of the Academy of Sciences of the USSR, Moscow, May 1991

Invited talk at 'Fezafest', a meeting in honor of Professor Feza Gürsey, at Yale University, May 1991

Two lectures at TASI '91, in Boulder, Colorado, June 1991

Invited talk at the IFT Workshop on Dark Matter, Gainesville, FL, Feb. 14-16, 1992

Invited talk at the ITP Workshop on Cosmological Phase Transitions, Santa Barbara, CA, April 2-4, 1992

Particle Physics Seminar at UCLA, May 8, 1992

Colloquium at the Institute for Theoretical Physics, Santa Barbara, CA, May 13, 1992

Particle Physics Seminar at Caltech, May 25, 1992

Seminar at ITP, Santa Barbara, CA, June 17, 1992

Colloquium at the FSU Physics Department, Tallahassee, FL, Oct. 8, 1992

Invited talk at the Coral Gables Conferences on Unification in the Large and the Small, Miami, FL, Jan. 26, 1993

Particle Theory Seminar at the Univ. of Pennsylvania, Philadelphia, PA, April 12, 1993

\section{Thorn}

Theoretical Physics Seminar, Imperial College, London, England, February 1991 Theoretical Physics Seminar, Durham University, Durham, England, February 1991

Theoretical Physics Seminar, Oxford University, Oxford, England, February 1991 
XVth Triangular Meeting (Utrecht, Rome, Paris), April 3-5, 1991

Theoretical Physics Seminar, Southhampton University, May 1991

Theoretical Physics Seminar, Queen Mary College, London, England, May 1991

DAMTP, Cambridge University, May 1991

First International Sakhavov Conference on Physics, Lobedev Institute, Moscow, USSR, May 27-31, 1991

Theoretical Physics Seminar, Karlsruhe, Germany, June 1991

LMS Durham Research Symposium, Conformal Field Theory and Related Topics, July $9 \cdot 19,1991$

Theoretical Physics Seminar, ENS, Paris, France, August 1991

Lecture to Symposium in Honor of Kenneth Johnson, Cambridge, Mass., November 1991

Theoretical Physics Seminar, Univ. of North Carolina, Chapel Hill, NC, March 1992

Theoretical Physics Seminar, Institute for Advanced Study, Princeton, NJ, June 1992

Theoretical Physics Seminar, Johns Hopkins University, Baltimore, MD, July 1992

Invited talk at the Coral Gables Conferences on Unification in the Large and the Small, Miami, FL, Jan. 26, 1993

Theoretical Physics Seminar presented at SLAC, June 1993

\section{Woodard}

Seminar, University of Florida, March 26, 1991

Seminar, 'University of Crete, June 24, 1991

Colloquium, University of Central Florida, Sept. 27, 1991

Seminar, University of Florida, Nov. 15, 1991

Seminar, University of Florida, Nov. 26, 1991

Seminar, University of Florida, March 31, 1992

Seminar, University of Florida, April 7, 1992

Seminar, University of Crete, June 23, 1992

Invited Talk, Rome-Paris-Utrecht Triangular Conference, July 29, 1992

Seminar, University of Florida, Sept. 18, 1992

Seminar, Brandeis University, Sept. 24, 1992

Seminar, Brown University, Sept. 25, 1992 


\section{B. Travel}

\section{Field}

SDC Collaboration Meeting, SSCL, Jan. 9-12, 1991

SDC Collaboration Meeting, Argonne Nat. Lab., March 20-23, 1991

SDC "Physics Subgroup" Meeting, SSCL, May 31-June 1, 1991

SDC Collaboration Meeting, Berkeley, August 5-10, 1991

URA Fermilab Review, Jan 17-18, 1992

Participated in the XXVI International Conference on High Energy Physics, SMU, Dallas, TX, August 6-12, 1992

URA Fermilab Review, February 5-6, 1993

SSC Laboratory, Dallas, TX, June 6-8, 1993

\section{Ramond}

University of Pennsylvania, May 1991

Johns Hopkins, May 1991

SSC Colloquium, May 1991

HEPAP, Washington, June 1991

XXth Conference in Differential Geometry in Physics, New York, June 1991

TASI, Boulder, Colorado, June 1991

Aspen Center for Physics, July 1991

John Hopkins Workshop, August 1991

University of Virginia, September 1991

University of Texas at Austin, September 1991

HEPAP travel and Witherell Subpanel member, 1992

Princeton University, May 1992

SSC, June 1992

Aspen Center for Physics, July 1992

Erice, Italy, Sept 1992

Ecole Normale, Paris, Sept 1992

Yale Workshop, Oct 1992

Coral Gables Conference, Jan. 1993

HARC, Houston, TX, April 1993

HEPAP travel, April, 1993

Johns Hopkins University, April 1993 
University of Chicago, May 1993

Southern Methodist University, May 1993

SSCC, Dallas, TX, May-June 1993

\section{Sikivie}

First International School Particles and Cosmology, Baksan Valley (Caucasus), USSR, May 1991

Institute for Nuclear Research, Moscow, May 1991

Fezafest, Yale University, May 1991

TASI '91, Boulder, Colorado, June 1991

Aspen, Colorado, June 1991

ITP, Santa Barbara, CA, April 2-4, 1992

ITP, Santa Barbara, CA, May and June, 1992

UCLA, May 8, 1992

Caltech, May 25, 1992

Aspen, July 6-26, 1992

FSU, Tallahassee, FL, Oct. 8, 1992

DPF92, Fermilab, Nov. 11-13, 1992

University of Maryland, Dec. 1-2, 1992

Coral Gables Conference, Miami, Jan. 24-27, 1993

Univ. of Pennsylvania, April 2, 1993

Thorn

Imperial College, London, February 1991

Durham University, Durham, England, February 1991

Oxford University, Oxford, England, February 1991

(Utrecht, Rome, Paris), France, April 1991

Southhampton University, May 1991

Queen Mary College, London, England, May 1991

DAMTP, Cambridge Univ., Cambridge, England, May 1991

Moscow, USSR, May 1991

Karlsruhe, Germany, June 1991

CERN, Geneva, Switzerland, July 1991

Durham, England, July 1991

ENS, Paris, France, August 1991 
MIT, Cambridge, Mass., November 1991

Chapel Hill, NC, March 1992

IAS, Princeton, NJ, June 1992

Johns Hopkins University, Baltimore, MD, July 1992

Coral Gables Conference, Miami, Jan. 24-27, 1993

Workshop at the Institute for Theoretical Physics in Santa Barbara, June 1993

Qiu

Cornell University, Ithaca, NY, August 1992

Woodard

University of Crete, 6/11/91-7/7/91

University of Central Florida, 9/27/91

University of Crete, 5/16/92-7/15/92

Brandeis University, 9/24/92

Brown University, 9/25/92-9/29/92 
Appendix A. GROUP PUBLICATIONS (since 1991)

1. The Graph Representation Approach to Topological Field Theory in 2+1 Dimensions, S. Martin, UFIFT-HEP-91-2, Int. J. Mod. Phys. A7, 563 (1992).

2. The String Difference Equation of $d=1$ Matrix Model and $W_{1+\infty}$ Symmetry of the KP Hierarchy, M. Awada and S. J. Sin, UFIFT-HEP-91-3, Int. J. Mod. Phys. A7, 4792 (1992).

3. Two Loop Calculations Using Nonlocal Regularization, G. Kleppe and $R$. Woodard, Ann. Phys. 221, 106-164 (1993).

4. Stringy Domain Walls and Target Space Modular Invariance, M. Cvetic, F. Quevedo and S. J. Rey, UFIFT-HEP-91-5, Phys. Rev. Lett. 67, 1836 (1991).

5. Superconformal Field Theory of Axionic Instantons and Axionic Strings, S. J. Rey, UFIFT-HEP-91-6, unpublished.

6. A Classical Theory of Continuous Spin and Hidden Gauge Invariance, D. Zoller, UFIFT-HEP-91-7, unpublished.

7. Dynamical Electroweak Symmetry Breaking with Top Quark and Neutrino Condensates, S. P. Martin, UFIFT-HEP-91-8, Phys. Rev. D44, 2892 (1991).

8. Semirigid Construction of Topological Supergravities, S. Rey, S. Govindarajan, and P. Nelson, UFIFT-HEP-91-10, Nucl. Phys. B365, 633 (1991).

9. Construction of Lax Operators from Weight Diagrams, S. Carbon and E. Piard, UFIFT-HEP-91-11, unpublished.

10. Supermatrix Models, S. Yost, UFIFT-HEP-91-12, Int. J. Mod. Phys. A7, 6120 (1991).

11. Metaplectons' and Fractional Statistics, D. Castaño, UFIFT-HEP-91-13, Phys. Lett. B269, 345 (1991).

12. A Higher Derivative View of the Arc Length and Area Actions, D. Zoller, UFIFTHEP-91-14, unpublished.

13. Top Quark and Higgs Mass Bounds from a Numerical Study of Super-GUTs, $H$. Arason, D. Castaño, B. Keszthelyi, S. Mikaelian, E. Piard, P. Ramond and B. Wright, UFIFT-HEP-91-16, Phys. Rev. Lett. 67, 2933 (1991).

14. Phase-Space Structure of Cold Dark Matter Halos, P. Sikivie and J. Ipser, UFIFTHEP-91-17, Phys. Lett. B291, 288-292 (1992).

15. Physical Green's Functions in Quantum Gravity, N. Tsamis and $R$. Woodard, UFIFTHEP-91-21, Ann. of Phys., 215, 96-155 (1992).

16. SDC Solenoidal Detector Notes: Jets in the Forward Region, R. D. Field, M. Barnett, and A. White, UFIFT-HEP-91-22, SDC-91-043 (1991).

17. Renormalizable Top-Quark Condensate Models, S. Martin, UFIFT-HEP-91-24, Phys. Rev. D45, 4283 (1992).

18. The Super $W_{1+\infty}$ Symmetry of the Manin-Radul Super KP Hierarchy, A. Das, E. Sezgin, and S. J. Sin, UFIFT-HEP-91-26, Phys. Lett. B277, 435 (1992). 
19. Weight Diagram Construction of Lax Operator, S. Carbon and E. Piard, UFIFTHEP-91-27, J. Math Phys. 33, 2664 (1992).

20. Renormalizability of $\mathrm{D}=3$ Topologically Massive Gravity, B. Keszthelyi and G. Kleppe, UFIFT-HEP-91-30, Phys. Lett. B281, 33 (1992).

21. Self-Dual Solitons in $2+1$ Dimensions Without the Higgs Mechanism, S. Mikaelian, UFIFT-HEP-91-32, unpublished.

22. Renormalization Group Study of the Standard Model and Its Extensions: I. The Standard Model, H. Arason, D Castaño, B. Keszthelyi, S. Mikaelian, E. Piard, P. Ramond and B. Wright, UFIFT-HEP-91-33, Phys. Rev. D46, 3945-3955 (1992).

23. Is There Graceful Exit Problem in the Old Inflationary Model?, J. Hong, P. Oh and S. Sin, UFIFT-HEP-92-1.

24. Symplectic Structure of Isospin Particles in Yang-Mills Fields, $P$. Oh, UFIFT-HEP92-2, Mod. Phys. Lett. A7, 1923 (1992).

25. Mass and Mixing Angle Patterns in the Standard Model and Its Minimal Supersymmetric Extension, $P$. Ramond, contributed to John Klauder's 60th Birthday, UFIFTHEP-92-4, unpublished.

26. Domain Wall Formation in Late-Time Phase Transitions, E. W. Kolb and Y. Wang, UFIFT-HEP-92-5, Phys. Rev. D45, 4421 (1992).

27. Cosmic Microwave Background Anisotropies From Plausible Double Inflation, J. Fry and $Y$. Wang, UFIFT-HEP-92-6, Phys. Rev. D46, 3318 (1992).

28. A Tumbling Top-Quark Condensate Model, S. Martin, UFIFT-HEP-92-7, Phys. Rev. D46, 2197 (1992).

29. Mass and Mixing Angle Patterns in the Standard Model and Its Minimal Supersymmetric Extension, H. Arason, E. Castaño, E. Piard and P. Ramond, UFIFT-HEP-928, Phys. Rev. D47, 322 (1993).

30. Effects of a Nambu-Goldstone Boson on the Polarization of Radio Galaxies and the Cosmic Microwave Background, D. Harari and P. Sikivie, UFIFT-HEP-92-9, Phys. Lett. B289, 67-72 (1992).

31. Wiggly Relativistic Strings, J. Hong, S. Kim and P. Sikivie, UFIFT-HEP-92-10, Phys. Rev. Lett. 69, 2611-2614 (1992).

32. Late Time Cosmological Phase Transition and Galactic Halo as Bose-Liquid, S. Sin, UFIFT-HEP-92-11.

33. Quark-Antiquark Regge Trajectories in Large $\mathrm{N}_{c} \mathrm{QCD}, C$. Thorn and $M$. McGuigan, UFIFT-HEP-92-12, Phys. Rev. Lett. 69, 1312 (1992).

34. Intermediate Scales of Symmetry Breaking in Calabi-Yau Models, M. Masip, UFIFTHEP-92-13, Phys. Rev. D46, 3601 (1992).

35. The Structure of Perturbative Quantum Gravity on a de Sitter Background, N. Tsamis and $R$. Woodard, UFIFT-HEP-92-14, to appear in Communications in Mathematical Physics.

36. An Analytic Description of Thick-Wall Bubbles, J. Hong, UFIFT-HEP-92-15. 
37. Enforcing the Wheeler-DeWitt Constraint the Easy Way, $R$. Woodard, UFIFT-HEP92-16, Class. and Quantum Grav. 10, 483-496 (1993).

38. The Sine-Gordon Model and the Small $\mathrm{K}^{+}$Region of Light-Cone Perturbation Theory, P. Griffin, UFIFT-HEP-92-17, Phys. Rev. D46, 3538 (1992).

39. Definition of Negative Heat Capacities for Classical Fluids, $R$. Woodard and P. Landsberg, UFIFT-HEP-92-18, to appear in J. Statistical Physics.

40. Staggered Fermions and Chiral Symmetry Breaking in Transverse Lattice Regulated QED, P. Griffin, UFIFT-HEP-92-19, Phys. Rev. D47, 3530 (1993).

41. Mode Analysis and Ward Identities for Perturbative Quantum Gravity in de Sitter Space, N. Tsamis and $R$. Woodard, UFIFT-HEP-92-20, Phys. Lett.B292, 269 (1992).

42. Explaining Cloud Chamber Tracks, A. Broyles, UFIFT-HEP-92-21.

43. Some Simple Criteria for Gauged R-Parity, S. Martin, UFIFT-HEP-92-22, Phys. Rev. D46, 2769 (1992).

44. Relaxing the Cosmological Constant, $R$. Woodard and N. Tsamis, UFIFT-HEP-92-23, Phys. Lett. B301, 483-496 (1993).

45. Strong Infrared Effects in Quantum Gravity, $R$. Woodard and N. Tsamis, UFIFTHEP-92-24, in preparation.

46. Massive Neutrinos in a Calabi-Yau Model, M. Masip, UFIFT-HEP-92-27, Phys. Rev D47, 3071 (1992).

47. Long Range Forces From Two Neutrino Exchange, P. Sikivie and S. Hsu, UFIFTHEP-92-28.

48. SDC Solenoidal Detector Notes: Forward Jets and Missing $E_{T}, R$. D. Field, UFIFTHEP-92-30, SDC-92-355 (1992).

49. Continuing Between Closed and Open Strings, C. B. Thorn and M. B. Green, Nuclear Phys. B367, 462 (1991).

50. Contractions of Lie Groups applied to Differential Equations, Marc de Montigny, UFIFT-HEP-92-31.

51. Third Quantization and Black Holes, M. McGuigan, UFIFT-HEP-92-32.

52. Casimir Forces Between Beads on Strings, E. D'Hoker and P. Sikivie, UFIFT-HEP92-33.

53. Axion Dection in the Milli-eV Mass Range, $P$. Sikivie, D. B. Tanner and $Y$. Wang, UFIFT-HEP-93-2.

54. Enchancing the Heavy Higgs Signal with Jet-Jet Profile Cuts, $R$. Field and $P$. Griffin, UFIFT-HEP-93-3, to appear in Phys. Rev. D.

55. SDC Solenoidal Detector Notes: Enchancing the Heavy Higgs Signal, $R$. Field and $P$. Grifin, UFIFT-HEP-93-4, SDC-93-459 (1993).

56. Baryogenesis in a Supersymmetric Model with $\mathrm{Z}_{3}$ matter parity, M. Masip and $Y$. Wang, UFIFT-HEP-93-5, to appear in Phys. Rev. $D$.

57. Stitching the Yukawa Quilt, $P$. Ramond, R. Roberts and G. Ross, UFIFT-HEP-93-6, to appear in Nucl. Phys. B. 
58. The de Sitter-Invariant Differential Equations and Their Contraction to Poincare and Galilei, M. de Montigny, UFIFT-HEP-93-10.

59. M. Masip and M. de Montigny, UFIFT-HEP-93-11.

60. Oscillator Representation of Sister Trajectories, S. Carbon and C. B. Thorn, UFIFTHEP-93-14.

61. Sister Trajectories and the Cerulus-Martin Bound, S. Carbon, UFIFT-HEP-93-15.

62. Sparticle Spectrum Constraints, S. Martin and P. Ramond, UFIFT-HEP- 93-16, submitted to Phys Rev. D.

\section{B. Conference Reports}

63. Two lectures on Neutrinos, $P$. Ramond, two lectures delivered at TASI '91, Boulder, Colorado, UFIFT-HEP-91-25, to appear in proceedings.

64. Reformulating String Theory with the 1/N Expansion, C. B. Thorn, UFIFT-HEP91-23, in Sakharov Memorial Lectures in Physics, L. V. Keldysh and V. Ya Feinberg, Eds., Nova Science Publishers, Inc. (1992), p. 447.

65. Neutrinology, P. Ramond, UFIFT-HEP-91-28, to appear in Proceedings of Johns Hopkins Workshop.

66. Axion Searches, P. Sikivie, UFIFT-HEP-91-29, in "Perspectives in the Standard Model", TASI 91, edited by R. K. Ellis et al., World Scientific, 1992, pp 399-418.

67. Neutrinos: Harbingers of New Theories, $P$. Ramond, UFIFT-HEP-91-34, to appear in Festschrift volume for E.C.G. Sudarshan.

68. Dark Maiter Axions, $P$. Sikivie, lecture given at the Dark Matter Workshop, Univ. of Florida, Feb. 12-14, 1992, UFIFT-HEP-92-25, to appear in the proceedings.

69. Neutrinos as Fundamental Probes, P. Ramond, UFIFT-HEP-92-29, to appear in the Proceedings of Daniel Challonge School.

70. Symmetric Textures, $P$. Ramond Invited talk at Global Foundation Conference, Coral Gales, Florida, Jan. 1993, UFIFT-HEP-93-7, to appear in the proceedings.

71. Regge Trajectories in QCD, C. B. Thorn, Invited talk at Global Foundation Conference on Symmetries in the large and in the small, Coral Gables, Florida, January 1993, UFIFT-HEP-93-12, to appear in the proceedings.

72. Renormalization Group Study of the Minimal Supesymmetric Standard Model: No Scale Models, $P$. Ramond, Invited talk at workshop "Recent Advance in the Superworld", Houston Advanced Research Center, the Woodlands, TX, April 1993, UFIFTHEP-93-13, to appear in the proceedings. 


\section{Research in Experimental High Energy Physics}

\section{Task B}

Principal Investigators:

Paul Avery and John Yelton

Physics Department

University of Florida

Gainesville, Florida 32611 


\section{Introduction}

UF Task B has been funded continuously by DoE since 1986. For the year 1991-92 it was decided to split the previous task into two, with J.K. Walker (hardware development for SSC experiments) splitting off into a separate task. In summer 1991 Andrew White left UF to be a Full Professor at University of Texas (Arlington) where he is establishing a new High Energy Experimental group and will continue to work with DO and SDC collaborations.

\section{1(a) Search for Senior Experimentalist}

A search is underway to hire a senior faculty member in High Energy Physics who will lead a new group directed towards SSC experiments. Well-known members of the HEE community have been invited to visit the campus (J. Friedman, M. Shocket, K. Berkelman, etc.), with a view to defining with the upper administration a package likely to attract the calibre of person we are trying to attract. This proposal does not address the funding that will be necessary when this position is filled. It is envisaged that this senior hire will be followed by several junior faculty positions.

\section{1(b) New Physics Building}

Funding for the new Physics Building has been unaffected by the continuing State financial crisis. A site has been chosen (half a mile from the present Physics buildings), and an architect has been selected. The new building will be large enough to house the entire Department and will include a $4000 \mathrm{ft}^{2}$ laboratory where it will be possible to build large scale HEE detectors. The funding for the building is greater than \$30M and it should be complete for occupancy in 1997-98.

\section{The CLEO Experiment}

\subsection{Introduction and History}

The CLEO experiment resides at the south end of the Cornell Electron Storage Ring (CESR). The detector is used to study $e^{+} e^{-}$interactions at center of mass energies near $10 \mathrm{GeV}$. Much of our effort has focused on the properties of the $Y$ family of resonances (a total of 6 have been discovered), particularly the $\Upsilon(4 S)$ since it decays into mesons carrying a single b quark. The energy range is also well suited to studies of charmed mesons and baryons. This has become an increasingly fruitful area of the research for CLEO now that the SPEAR facility as SLAC has been closed down. The CLEO collaboration now comprises around 200 professional physicists 
and graduate students from 19 U.S. institutions ( Caltech, Carnegie-Mellon, Colorado, Cornell, Florida, Harvard, Illinois, Kansas, Minnesota, Ohio State, Oklahoma, Purdue, Rochester, San Diego, Santa Barbara, SUNY-Albany, Southern Methodist University, Syracuse, and Vanderbilt). CLEO has now become an International institution as we have been joined by a large Canadian contingent). In the last few years CLEO has grown from a mostly NSF funded collaboration based in the North-East of the U.S.A. into an NSF and DoE funded national collaboration. In the future it appears that there will be more support from the DoE and also from outside the country. Florida has been a member since September, 1985

CLEO completed a $\$ 36$ million upgrade program funded by NSF in 1989. The 1987-88 data set was taken with a new tracking system (main drift chamber and vertex chamber), this configuration was known as "CLEO 1.5". Since then there have been further improvements in the inner detector together with a large "crystal calorimeter" which dramatically improves the detection and measurement of neutral particles. The detector, in its new and improved form, is known as "CLEO II". The detector has been running in CLEO II for almost two years. In 1992 and 1993 many papers have been published that take advantage of the crystal calorimeter. In the meantime, upgrades to CESR have led to large increases in luminosity. The data sample analyzed to date is approximately five times the size of the CLEO 1.5 data sample.

\subsection{Summary of Work at CLEO: Oct. 1992 - Sep. 1993}

\section{2a Physics Analysis at CLEO}

All the CLEO analysis done now uses the CLEO II configuration. For two years now, CESR has settled into its usual cycle of running on $\Upsilon(4 s)$ and just below the $\Upsilon(4 s)$ to optimize $B$ meson studies. Around $2.5 \mathrm{fb}^{-1}$ has now been taken and is being analyzed. Some published analyses have used $1.9 \mathrm{fb}^{-1}$. The full analysis of the data is now possible a few months after the data has been taken.

Although the collaboration has expanded greatly over the last few years, there are areas which need more people support. First, there is a huge amount of new data to be processed and fully analyzed, which in turn requires a large amount of computer CPU and disk. Second, the Inner Detector Task Force (IDTF) was launched in 1991 with a goal to completely rebuild the charged particle capabilities of CLEO II and upgrade CESR to accommodate a new luminosity of $10^{33}$, about 5 times the present luminosity. Third, there is a large effort to get a complete rebuilding of the CESR machine (i.e. a "B-Factory"). Many of the new collaborators work primarily on this project and the associated detector modifications. Finally, the running of the 
detector requires two people on shift, 24 hours a day, and of course a lot of hard work to keep all aspects of harware fully operational.

In the last nine months, since our last proposal, the following papers have appeared in journals.

1. Production and Decay of the $D_{s}(2536)$. J. Alexander et al., Phys. Lett. B303, 477.

2. A Search for Exclusive $\tau \rightarrow \eta$. A. Bean et al., PRL 70, 138.

3. $\tau$ Decays with one CHarged Particle plus Multiple $\pi^{0}$ s. M. Procario et al., PRL 70:1207.

4. Measurement of the $\tau$-lepton Electronic Branching Fraction. D. Akerib et al., PRL 693610.

5. Lepton Asymmetry Measurement in $\bar{B} \rightarrow D^{+} l v$; Implications for V-A. S.Sanghera et al., Phys. Rev. D47 791 (1993).

6. Measurement of the $D^{*}(2010)$ Branching Fraction. F. Butler et al., PRL 69, 2041 (1992).

7. A Shape Study of Quark Jets versus Gluon Jets at $10 \mathrm{GeV}$. J. Alexander et al., Phys. Rev. D.

In addition the following are available as preprints (CLNS reports).

1. Study of the Decays $\Lambda_{c}^{+} \rightarrow \Xi^{0} K^{+}, \Lambda_{c}^{+} K^{+} K^{-}$and $\Xi^{-} K^{+} \pi^{+}$. P. Avery et al., CLNS-93-1205. This analysis was performed by Florida.

2. A Limit on the $\psi_{\tau}$ neutrino mass. D. Cinabro et al., CLNS-93-1195.

3. Measurement of the $\tau$-lepton Mass. R. Ballest et al., CLNS-93-1194.

4. Two Measurements of $B^{0} B$ Mixing. J. Bartelt et al., CLNS-93-1207.

5. Study of $D^{0}$ Decays into $K^{0} K^{0}$. M Procario et al., CLNS-92-1167.

6. Evidence for Penguins. First Observation of $B \rightarrow \mathrm{K}^{*} \gamma$. R. Ammar et al., CLNS-93-1212.

In addition the following are available as CLNS notes and have been sent for publication. 


\section{3a Physics analyses}

Florida has made a contribution to the physics analysis of CLEO data in the last few years out of all proportion to its size. Inevitably all CLEO publications have an author list including all the collaborators. The following papers published in the last few years were based on work done by UF. In most cases the analysis work for these papers were performed entirely by members of the UF group (the present members together with $D$. Besson, now a faculty member at $U$. of Kansas, during his stay with us as a post-doc). In some cases a member of our group contributed part of the paper.

1. Limits on Rare B Decays. P.Avery et al., Phys. Lett. B183,429 (1987).

2. Production of $\eta$ and $\omega$ mesons in $\tau$ decays. P. Baringer et al., PRL 59, 1993 (1987).

3. Search for the Charmless Decays C.Bebek et al., B $\rightarrow \bar{p} \bar{p} \pi$ and $\mathrm{p} \overline{\mathrm{p}} \pi \pi$. PRL 621,8 (1989).

4. $\Sigma_{\mathrm{c}}^{++}$and $\Sigma_{\mathrm{c}}^{0}$ Production in $\mathrm{e}^{+} \mathrm{e}^{-}$Annihilation. T. Bowcock et al., PRL 62,1240 (1989).

5. A Search for Exclusive Penguin Decays in B Mesons. P. Avery et al., Phys. Lett. B233, 470 (1989).

Observation of the Charmed, Strange Baryon $\Xi_{C}^{0}$ P. Avery et al., PRL 62, 863 (1989).

6. Measurement of the Isospin Splitting $\Xi_{c}^{+} \Xi_{c}^{0}$ M.S. Alam et al., Phys. Lett. B226, 401 (1989).

7. Study of D Decays into KR and $\pi \pi^{-}$. J. Alexander et al., PRL 65, 1184 (1990).

8. Study of $K^{*}$ Production in $\tau$ Decays. M. Goldberg et al., Phys. Lett. B 223 (1990).

9. The Decay $D^{0} \rightarrow K^{0} R^{0}$. J. Alexander et al., PRL65, 1184 (1990).

10. radiative $\Upsilon(1 \mathrm{~s})$ Decays. R. Fulton et al., Phys. Rev. D41, 1401 (1990).

11. Study of $D^{0}$ Decays into final states including a $\pi^{0}$ or an $\eta^{0}$. K.Kinoshita et al., Phys. Rev. D43, (1991).

12. Unusual Decays of D Mesons. R.Ammar et al., Phys. Rev. D44, 3383 (1991).

13. $\Lambda_{c}^{+}$Production in $\mathrm{e}^{+} \mathrm{e}^{-}$Annihilation at $\mathrm{E}=10 \mathrm{GeV}$. P. Avery et al. Phys. Rev. D43, 3591 (1991).

14. Observation of the Decay $\Xi_{\mathrm{c}}^{0} \rightarrow \Omega^{-} \mathrm{K}^{+}$. Phys. Lett. B283, 161 (1992).

The above list describes only the work that has actually been published. There is of course a lot of work in progress that has not yet reached that stage.

Jorge Rodriguez and Paul Avery are working with Cornell's Tom Browder in searching for two-body "color suppressed" decay of B mesons, i.e., modes which are expected to be suppressed (naively) by a factor of 9 in the rate because of color mismatch in forming the 2 body final state. Jorge has concentrated on the exclusive reconstruction of decays to a $D$ or a $D^{*}$ meson. The analysis includes measurement of the decay rates to the non-color suppressed modes 
used for normalization. Although no signals for color-suppressed modes have been found, the limits set are a challenge for theorists. We also presented this year an accurate measurement of $a_{2} / a_{1}$, the ratio of Bauer-Stech-Wirbel parameters which determines the actual degree of color suppression, using decay modes in which color suppressed and color unsuppressed diagrams interfere. Our measurements show unambiguously that the sign of the ratio is positive, in disagreement with theoretical predictions. These results were presented at the Washington APS and at DPF in November 1992 and we expect to publish them during the coming year. The work is part of a large Physical Review D publication that is presently in the final stages of preparation.

John Yelton found a new way to increase the signal:background ratio in hyperons decaying to $\pi^{0}$ 's, such as $\Sigma^{+}$'s and $\Xi^{0}$ 's. This has led to the possibility of finding new charmed baryon decay channels and is used by all members of the charmed baryon group at CLEO. He has written the paper on decays of the $\Lambda_{\mathrm{c}}^{+}$into $\Sigma^{+} \mathrm{K}^{+} \mathrm{K}^{-}$and $\Xi^{0} \mathrm{~K}^{+}$. This was submitted to the Dallas conference. It was, then extended to include a new measurement of $\Lambda_{\mathrm{C}}^{+} \rightarrow \Xi^{-} \mathrm{K}^{+} \pi^{+}$and resonant substructure. Decays of this form are particularly interesting because they indicate that decays due to $\mathrm{W}$-exchange mechanisms are important for charmed baryon. The analysis has been sent to PRL and we are awaiting the referee's comments. He has also performed searches for $\Omega_{c}$ 's. In particular he has obtained a limit for $\Omega_{c} \rightarrow \Xi^{-} \mathrm{K}^{-} \pi^{+} \pi^{+}$that is far lower than the published ARGUS signal. He is hoping to put this together with other $\Omega_{c}$ searches for a paper for the summer conferences.

He has also made measurements of some large, but mundane, decay modes of the $\Lambda_{c}^{+}$, in particular those involving a proton along with a $\mathbb{R}$. This work has been presented to the Collaboration and a summer conference paper is being written.

Ransom Stephens has been looking $\mathrm{a} b \rightarrow \mathrm{u}$ transitions using $\mathrm{B} \rightarrow \mathrm{D}_{\mathrm{s}} \mathrm{X}$ and $\mathrm{D}_{\mathrm{s}}^{*}$ where $\mathrm{X}=\pi, \rho, \omega$, or $\mathrm{a}_{1}$. There are good theoretical reasons to believe these decays will exist at a measurable rate. The analysis also involves searching for the unusual $b \rightarrow c$ decays of the form $B \rightarrow D_{S}^{(*)} K^{(*)}$, and the normalization modes of the form $B \rightarrow D_{s} D$. He has as yet seen no signal for $b \rightarrow u$, but has been able to place limits on these decays that are far more stringent than anyone previously. The results are being combined to give limits on the ratio of the $\mathrm{KM}$ matrix elements $\mathrm{V}_{\mathrm{ub}} / \mathrm{V}_{\mathrm{cb}}$ which are surprisingly competitive with the limits obtained from the exclusive semi-leptonic measurements. The results are in disagreement with the Argus results presented at the Dallas conference. He gave a talk on behalf of CLEO at the DPF conference at Fermilab, and a publication is planned. Ransom presently has an offer of a postion as Assistant Professor at UT Arlington. If he chooses to accept the offer he will finish his analysis with CLEO before moving and we will immediately move to fill the vacancy. 
Ame Freyberger is preparing a paper on the measurement of ratio of the $\left(D^{+} \rightarrow \pi^{0} l v\right)$ to $\mathrm{D}^{+} \rightarrow \rightarrow \mathrm{K}^{0} \mathrm{lv}$. This is difficult analysis because the decays do not appear as bumps in mass plots, but rather areas under background subtracted pseudomass difference plots. The result is a ratio, $R$, of $8.5 \pm 2.7 \pm 1.4 \%$. From this he has extracted a ratio of form factor $f_{\pi} / f_{K}=1: 3 \pm 0.2 \pm 0.1$ in agreement with model predictions. This analysis has just been sent of to Physical Review Letters.

Ame is applying this technique to make a new measurement of the absolute branching fraction of $D^{0}$ to $\mathrm{K} \pi$ using the angular correlation between the $\pi^{+}$from the $\mathrm{D}^{*+}$ decays and the event jet axis. The result has a statistical precision of $2 \%$, with a systematic precision of $4 \%$. He has then extended this technique to include the slow $\pi^{0}$ 's from $D^{*_{0}} \rightarrow D^{0} \pi^{0}$ and $D^{*} \rightarrow D^{+} \pi^{0}$, and a measurement to obtain a measurement of the $\mathrm{D}^{+} \rightarrow \mathrm{K}^{-} \pi^{+} \pi^{+}$. The precision of this measurement is about $10 \%$ which improves our knowledge of the $\mathrm{D}^{+}$branching ratio. Both these results have been approved for conference presentation and drafts of papers, intended for Physical Review Letters, are in the process of being written.

Ame has also used a sample of $200 \mathrm{D}^{+} \rightarrow \mathrm{K}^{0} \mathrm{e}^{+} \mathrm{V}$ events to make a measurement of the $\mathrm{D}^{+}$ $\rightarrow \mathrm{K}^{*_{0}} \mathrm{e}^{+} \mathrm{v} / \mathrm{D}^{+} \rightarrow \mathrm{K}^{0} \pi^{+}$ratio of branching fractions. This work is being included with CLEO measurements of $D^{0} \rightarrow K^{*} e v$ and $D^{+} \rightarrow K^{*} e^{+} v$ and represents the first time that a single experiment has made all four of these measurements. These results are being prepared for the "summer" conference season, and a draft being prepared for submission to Physics Letters B shortly.

He has further plans to measure the inclusive $D^{0} \rightarrow$ Xev branching ratio, once again using the technique of determining the number of $D^{*+} \rightarrow D^{0} \pi^{+}$decays from the slow $\pi$ - jet axis angle. Preliminary studies imply that a statistical precision of around $4 \%$. This results, coupled with other CLEO measurements, will be precise enough to determine if the measured exlusive modes saturate the inclusive measurement.

$\mathrm{He}$ has also been working with $\mathrm{Xu} F u$, an Oklahoma University student, on the measurement of $B^{0} \rightarrow D^{*}+l^{-} v$ branching fraction and an extraction of the $\mathrm{KM}$ matrix element $\mathrm{V}_{\mathrm{cb}}$. This result has been presented at the APS meeting and is the most precise to date. A draft is being prepared for the summer conferences.

Paul Avery has written track fitting software to include the Kalman filter technique which allows the inclusion of $\mathrm{dE} / \mathrm{dx}$ and multiple scattering effects in the fit. This is expected to improve the tracking errors considerably, especially for soft tracks. The goal is to get this new fitter into the standard data reduction by Fall, 1993. In addition, he is working on a method which would allow tracks to be refit from data summary files, thus allowing for much more sophisticated kinds of analyses. He has also worked with the Inner Detector Task Force by providing track fitting and simulation tools to compare tracking requirements using different 
designs involving silicon layers with various outer tracking chamber designs. He also wrote a large kinematic fitting package (to improve resolution) which has already been used in several analyses and in B factory work. He also serves on the speakers committee which selects speakers for upcoming conferences. He gave talks at the SLAC B factory meeting in April, 1992, chaired a computing review panel at the SSCL in Oct. 1992, and was a member of the SSC Computing Planning Committee in 1993.

\section{3b CLEO II Pass 2 data reduction}

We have already converted all our code to run on our large UNIX computing system, including using the UFMULTI multi-processing package to analyze the large CLEO II data sample. We have used our UNIX power extensively to run the PASS 2 processing (i.e. event reconstruction). In 1991 we had visitors at Cornell to help set up the processing chain here. Then Jorge Rodriguez took responsibility to complete the task, with help from student programmers and of course our system's manager. In 1992, Ransom Stephens worked with Rodriguez in the task of "recompressing" the large data sample already collected. this reprocessing of the data was necessary because many of the main analysis programs, including the track-finding and fitting, were not fully optimized before the first compress. In all almost $1 \mathrm{fb}^{-1}$ of data were analyzed in this fashion.

\section{3c Standard Monte Carlo generation}

Jorge Rodriguez, Ransom Stephens and John Yelton have been generating Monte-Carlo data locally using the GEANT description of the CLEO II apparatus. CLEO now has a MonteCarlo coordinator based at Cornell, he finds out what Monte-Carlo generation is wanted, and asks a local coordinator to generate the events. They then get taken by tape back to Cornell to be available for all users. In the month of May 1993, we generated 300,000 events for general CLEO use, and this rate can be kept up for long periods of time as necessary. In addition all the members of the group are actively pursuing research topics that require large samples of $M C$ data (the color suppressed B modes, for example, involve 18 decay modes).

\section{3d CLEO Librarian}

Arne Freyberger spent the year until September 1992 as the CLEO librarian. He was responsible for collecting together all additions and corrections to the CLEO libraries, and installing them in the CLEO system. This code then has to be tested, and if necessary debugged. The task is made more difficult by the fact that much of the code has to have different versions for VAXstations, DECstations and SGI machines. His performance as librarian won praise from many CLEO members not only for his conscientiousness in carrying out his duties but also the 
fact that he improved the system for updating libraries so that future generations of librarians would have a more foolproof system.

In the Summer of 93 he takes over as the "PTA secretary", that is the coordinator, for the charm semi-leptonic analysis group within CLEO.

\section{3e CLEO B Factory work}

We have not been doing much work on this since the Happer letter. However, an R\&D collaboration is starting up under the direction of Marvin Goldberg at Syracuse to raise international interest in a B factory at CLEO. We expect to be involved in this effort during the coming year but it is too early to tell yet what is needed to be done. It's likely that we will be involved in tracking and studying physics processes requiring good particle ID, such as the possible $C P$ violating decay $B-->D^{0} K$, where the $D^{0}$ decays into $C P$ eigenstates.

A very has been attending workshops and giving talks on $\mathrm{B}$ factories during the past year. He summarized the Cornell efforts at the SLAC workshop in April, 1992 and gave the experimental session summary at the same meeting. He also gave a colloquium at Indiana University (Feb. 1992) and attended the Cornell B factory workshop (Oct. 8-9, 1992).

\section{3f CLEO software}

UF has written a large fraction of the physics software that has become the standard for CLEO analysis in the past year.

\section{$2.3 \mathrm{~g}$ The UFMulti project}

The UFMulti software system we developed at UF has been the cornerstone of our computing effort since 1988. With UFMulti a single user can use the entire computing capability on a network, even when it is in the form of individual workstations. Our development of UFMulti also helped generate funding for computer resources and enabled us to negotiate better discounts from computer vendors.

In the past year we (Paul Avery and Chandra Chegireddy) have been working on a new version of UFMulti which is considerably more general than the previous one. We have done this work in collaboration with Prof. Theodore Johnson of the Computer Science Department and his students John Brothers,

Krishna Harathi, Jagadeesh Kasaraneni and Aric Zion. We are planning to use this new version of UFMulti first in CLEO physics analysis and then in the standard data reduction some time in 1993. 
Avery has given talks on UFMulti at the ICHEP meeting in Dallas (Aug. 1992) and at the DPF meeting in Chicago (Nov. 1992). Although his talk was approved at the CHEP92 meeting in Annecy this year he did not attend because of a lack of time. He has also given seminars (U of Illinos, Indiana U.) and colloquia (LSU and UF Computer Science Dept.). There is a further discussion of UFMulti in the Task $S$ proposal.

\section{3h Other activities}

Paul Avery chaired a panel that was convened in Oct. 1992 to assess the general performance of the Physics/Detector Simulation Facility (PDSF) at the SSCL, a 4000 MIPS facility that is the primary computing resource of SDC and GEM. He was also a member of the SSC Computer Planning Committee II in 1993. 
Task $C$

\author{
SECOND-GENERATION \\ DARK-MATTER AXION SEARCH \\ P. Sikivie, N. S. Sullivan, D. B. Tanner \\ Department of Physics, University \\ of Florida, Gainesville, FL 32611
}

\begin{abstract}
The axion appears in two of the most important questions in all of science, namely the origin of $\mathrm{CP}$ symmetry in the strong interactions, and the composition of the dark-matter of the universe. First predicted to exist in 1978, present laboratory, astrophysical, and cosmological constraints suggest axions have a mass in the $1 \mu \mathrm{eV}-1 \mathrm{meV}$ range. Axions are especially significant as dark matter if their mass is around $1 \mu \mathrm{eV}$. These dark matter axions may be detected by their coupling to photons through the $\mathbf{E} \cdot \mathbf{B}$ interaction in a tunable high- $Q$ microwave cavity permeated by a strong external magnetic field. Two pilot experiments have already reported results from this mass region, but fall short of making a definitive measurement by 3 to 4 orders of magnitude in power sensitivity. There is presently under construction a scaled-up experiment that would for the first time reach be sensitive enough to detect axions, given certain favorable choices of parameters. This second-generation experiment will also be the first to use multiple cavity arrays and will serve as a testbed for developments in axion detectors.
\end{abstract}




\section{TABLE OF CONTENTS}

\section{Page}

Abstract

I. Introduction . . . . . . . . . . . . . . . . . . 1

II. Axion physics . . . . . . . . . . . . . . . . 3

A. The axion as a solution to the strong CP problem . . . . . . . . 3

B. Axion properties . . . . . . . . . . . . . . . . . 4

C. Cosmological and astrophysical constraints on the axion mass . . . . . . . 5

D. The axion as a dark matter candidate . . . . . . . . . . . . . 7

III. The cavity detector of galactic halo axions . . . . . . . . . . . . . 8

A. The detector principle . . . . . . . . . . . . . . 8

B. The sensitivity as a function of search rate . . . . . . . . . . . 9

C. The optimization of cavity design . . . . . . . . . . . . . . . 11

IV. Summary of previous cavity searches . . . . . . . . . . . . . . . 12

A. The RBF experiment . . . . . . . . . . . . . . . . . . . . 12

B. The UF experiment . . . . . . . . . . . . . . . . 13

1. Magnet . . . . . . . . . . . . . . . . 13

2. Cavities . . . . . . . . . . . . . . . . . . . 13

3. Microwave system . . . . . . . . . . . . . . . . . . . . . . . . . 17

4. Spectrum analyzer . . . . . . . . . . . . . . . . . . . . . . 19

5. Results . . . . . . . . . . . . . . . . . 20

V. The second generation experiment . . . . . . . . . . . . . . . 22

A. 8.5 Tesla Magnet . . . . . . . . . . . . . . . . . . . . . . 22

B. Cavities . . . . . . . . . . . . . . . . . . . 22

1. Cavity design . . . . . . . . . . . . . . . . . 22

2. Cavity fabrication . . . . . . . . . . . . . 25 
3. Cavity tuning . . . . . . . . . . . . . . . . . . . . . . 26

4. Cavity Tolerances . . . . . . . . . . . . . . . . . . . . . . . . . 28

5. Tuning and combining cavity outputs . . . . . . . . . . . . . . . 28

C. Electronics . . . . . . . . . . . . . . . . . . . . . 31

1. Cryogenic amplifier . . . . . . . . . . . . . . . . . . 31

2. Receiver . . . . . . . . . . . . . . . . . . . 31

D. Search rate . . . . . . . . . . . . . . . . . . . . 32

References............................. 33 


\section{INTRODUCTION}

Measurements of the rotation speeds of stars in galaxies as a function of distance from the galactic center, have shown a universal feature: the speed is approximately constant for distances outside the core of the galaxy. This and other observations imply the existence of large halos of nonluminous matter surrounding galaries. The origin of this dark matter is a mystery whose solution is one of the most exciting challenges confronting science today. It seems probable from the success of models of nucleosynthesis in explaining the abundance of light isotopes, and of cosmological inflation in explaining the flatness and homogeneity of the present universe that the dark matter is non-baryonic. The leading non-baryonic candidates are neutrinos, weakly interacting massive particles such as the lightest supersymmetric partner (photino, higgsino, etc.), primordial black holes, or axions. Of all the non-baryonic candidates, the axion is the only particle for which a laboratory experiment can be carried out with current technology that could definitely detect the particle at the expected level of abundance.

The existence of the axion is well motivated from a particle physics point of view because it would explain why the strong interactions conserve parity $P$ and the product $C P$ of charge conjugation with parity. With regard to studies of large scale structure and galaxy formation, the axion falls in the category called "cold dark matter," whereas the neutrino is an example of "hot dark matter." Generally speaking, studies of large scale structure and galaxy formation favor cold dark matter over hot dark matter. If the dark matter is a mixture of the two, it is the cold component that constitutes the galactic halos.

Fortunately, the hypothesis that the halo of our galaxy is made of axions can be tested experimentally by looking for the resonant conversion of galactic halo axions to photons in a laboratory magnetic field. This document is a progress report of the University of Florida research in a "second-generation" axion search experiment that presently is in the construction phase. The axion detector, located at Lawrence Livermore National Laboratory, will improve the sensitivity over the first-generation detector that was built and operated at Florida by at least a factor of 50. The detector consists of a large superconducting magnet containing one or more microwave cavities; axions which overlap the high-field region will be stimulated to decay into microwave photons when the resonant frequency of the cavity equals the mass of the axion. The tuning range of the detector will be 1.5-11 $\mu \mathrm{eV}$; its sensitivity will make it the first dark matter detector of any type with enough sensitivity actually to detect a signal given the constraints on dark matter density set by astrophysical and cosmological considerations.

Pilot experiments at Brookhaven (collaboration with Fermilab and Rochester) and at the University of Florida have demonstrated the feasibility of most parts of the detector. Extremely sensitive microwave amplifiers, with noise temperatures in the required $1 \mathrm{~K}$ 
range, are now available. In addition the high-Q cavities, effective tuning mechanism, online spectral analysis technique, and efficient data acquisition and control system which are necessary for the experiment have been demonstrated. These results have encouraged us to take the next step: a substantially scaled-up detector employing a larger magnet, more complicated cavity arrangements, and possibly more sensitive amplifiers.

The experiment is a collaboration among several groups, each of which has key roles to play. Livermore has primary responsibility for the magnet and cryostat. The Institute for Nuclear Research of the Russian Academy of Sciences (INR) will construct the high-Q cavities. UF and MIT will provide the microwave electronics, will provide facilities for test of detector components, and will also explore new detector concepts. Additional collaborators are at the University of California Berkeley, Lawrence Berkeley Laboratory, Fermi National Accelerator Laboratory, and the Universty of Chicago.

It is our view that the axion experiment is extremely well motivated, with importance both for elementary particle physics and for astrophysics. The recently released long-range plan "The Decade of Discovery in Astronomy and Astrophysics," commissioned by the National Research Council, ${ }^{1}$ noted the importance of the dark-matter problem in general and the axion in particular. 


\section{AXION PHYSICS}

\section{A. The axion as a solution to the strong CP problem}

The axion ${ }^{2-4}$ was postulated fifteen years ago to explain why the strong interactions conserve $\mathrm{P}$ and $\mathrm{CP} .{ }^{5}$ Consider the Lagrangian of $\mathrm{QCD}$ :

$$
\mathcal{L}_{Q C D}=-\frac{1}{4} G_{\mu \nu}^{a} G^{a \mu \nu}+\sum_{j=1}^{n}\left[\bar{q}_{j} i \not D q_{j}-m_{j} q_{L j}^{\dagger} q_{R j}-m_{j}^{*} q_{R j}^{\dagger} q_{L j}\right]+\frac{\theta g^{2}}{32 \pi^{2}} G_{\mu \nu}^{a} \tilde{G}^{a \mu \nu}
$$

where the $G_{\mu \nu}^{a}$ are the gluonic field strengths, and $g_{i}, m_{i}$ are the quark fields and masses. The last term is a 4-divergence and hence does not contribute in perturbation theory. It does however produce non-perturbative effects ${ }^{5}$ associated with QCD instantons. ${ }^{6}$ These non-perturbative effects explicitly break the $U_{A}(1)$ quark flavor symmetry and thus solve the $U_{A}(1)$ problem, ${ }^{7}$ i.e., the problem why there is no light pseudo-Nambu-Goldstone boson similar to the pions produced by the spontaneous breaking of $U_{A}(1)$. Its solution is generally considered one of QCD's main successes. However, the very non-perturbative effects which solve the $U_{A}(1)$ problem make the physics of $Q C D$ depend upon the parameter $\theta$ which appears in Eq. 1. This creates, as we are about to describe, a new puzzle called "the strong CP problem."

Using the statement of the Adler-Bell-Jackiw anomaly of the $U_{A}(1)$ current, one can readily show that the physics of QCD depends upon $\theta$ only through the combination of parameters

$$
\bar{\theta}=\theta-\operatorname{argdet} m_{q}=\theta-\arg \left(m_{1} m_{2} \ldots m_{n}\right)
$$

If $\bar{\theta} \neq 0, Q C D$ violates $P$ and CP. The absence of $P$ and $C P$ violations in the strong interactions therefore places an upper limit upon $\bar{\theta}$. The best constraint comes from the present experimental upper limit on the neutron electric dipole moment. It yields: ${ }^{8,9}$

$$
\bar{\theta} \lesssim 10^{-9}
$$

We are then faced with the question: why is $\bar{\theta}$ so small? This is the strong CP problem. Recall that in the standard model of the strong and electroweak interactions, the quark masses originate in the electroweak sector of the theory. This sector must violate $\mathrm{P}$ and $C P$ to produce the correct weak interaction phenomenology, in particular $K_{L} \rightarrow 2 \pi$. There is no reason in the standard model to expect the overall phase of the quark mass matrix to exactly match the value of $\theta$ from the QCD sector in order to set $\bar{\theta}<10^{-9}$. In particular, if CP violation is introduced in the manner of Kobayashi and Maskawa, ${ }^{10}$ the Yukawa couplings that give rise to the quark masses are arbitrary complex numbers and hence arg det $m_{q}$ and $\bar{\theta}$ have no reason to take on any special value at all.

The most widely accepted solution to the strong CP problem is one proposed by Peccei and Quinn. ${ }^{2}$ They postulated the existence of a global $U(1)$ symmetry, now usually referred to as the $U_{P Q}(1)$ symmetry, which has the following properties: 
1. it is a symmetry of the classical theory, i.e., a symmetry of the theory at the Lagrangian level,

2. it is broken by the non-perturbative QCD effects which solve the $U_{A}(1)$ problem and make the physics of $Q C D$ depend upon $\theta$,

3. it is broken spontaneously by the vacuum expectation value of some scalar field.

The axion ${ }^{3}$ is the pseudo-Nambu-Goldstone boson associated with the spontaneous breaking of the $U_{P Q}(1)$ symmetry. One can show that if the standard model is augmented with a $U_{P Q}(1)$ symmetry, the parametir $\bar{\theta}$ becomes a dynamical variable. Its value depends upon the expectation value of the axion field $a(x)$, as follows:

$$
\bar{\theta}=\theta-\Delta-a(\mathbf{x}) / f_{a} .
$$

Here, $\Delta$ is a phase that depends upon the Yukawa couplings in the theory, and $f_{a} \equiv v_{a} / N$ where $v_{a}$ is the magnitude of the vacuum expectation value which breaks the $U_{P Q}(1)$ symmetry and $N$ is an integer which expresses the color anomaly of $U_{P Q}(1)$. It can be shown that no matter what are the values of $\Delta$ and of the other parameters in the theory, the expectation value of $a(x)$ sets $\bar{\theta}$ equal to zero (up to corrections of order $10^{-14}$ or smaller due to the fact that $\mathrm{CP}$ is not exactly conserved by the weak interactions). The strong CP problem is thus solved by postulating a $U_{P Q}(1)$ symmetry and the concomitant axion.

\section{B. Axion properties}

The axion is a light pseudo-scalar particle whose properties can be derived by using the methods of current algebra. ${ }^{3,11}$ Its mass is given by:

$$
m_{a}=\frac{f_{x} m_{\pi}}{f_{a}} \frac{\sqrt{m_{u} m_{d}}}{m_{u}+m_{d}} \simeq 0.6 \mathrm{eV}\left(\frac{10^{7} \mathrm{GeV}}{f_{a}}\right)
$$

where $f_{a}$ is the same quantity as appears in Eq. 4. As befits a Nambu-Goldstone particle, the axion couplings are all inversely proportional to $f_{a}$ or, in consideration of Eq. 5, proportional to its mass. In particular this is true of the coupling which is relevant to the experiment that we propose, i.e., that of the axion to two photons:

$$
\mathcal{L}_{a \gamma \gamma}=g_{\gamma} \frac{\alpha}{4 \pi} \frac{a}{f_{a}} F_{\mu \nu} \tilde{F}^{\mu \nu} .
$$

Here $\alpha$ is the fine structure constant and $g_{\gamma}$ is given in terms of the axion model parameters by

$$
g_{\gamma}=\frac{1}{2}\left(\frac{N_{e}}{N}-\frac{5}{3}-\frac{m_{d}-m_{u}}{m_{d}+m_{u}}\right)
$$

where $N=\operatorname{Tr}\left(Q_{P Q} Q_{c o l o r}^{2}\right)$ and $N_{e}=\operatorname{Tr}\left(Q_{P Q} Q_{e m}^{2}\right)$. "Tr" represents the sum over all left-handed Weyl fermions. $Q_{P Q}, Q_{e m}$ and $Q_{c o l o r}$ are respectively the Peccei-Quinn charge 
(the generator of the $U_{P Q}(1)$ symmetry), the electric charge and one of the generators of $S U^{c}(3)$. In all grand-unified models which obtain the (almost) successful Georgi-QuinnWeinberg ${ }^{12}$ prediction of $\sin ^{2} \theta_{w}$, one has $N_{e}=8 N / 3$ and hence $g_{\gamma}=m_{u} /\left(m_{u}+m_{d}\right) \simeq 0.36$. This is the case in particular of the Dine-Fischler-Srednicki-Zhitnitskii (DFSZ) model. ${ }^{13}$ In other models, one may have different values for $g_{\gamma}$ however. In particular, in the KimShifman-Vainstein-Zakharov (KSVZ) model, ${ }^{14}$ one has $N_{e}=0$ and hence $g_{\gamma} \simeq-0.97$.

When the axion was first proposed, it was thought that the breaking of $U_{P Q}(1)$ occurred at the electroweak scale and hence that $f_{a}$ is of the order of $250 \mathrm{GeV}$. The corresponding axion was searched for in various laboratory experiments ${ }^{4}$ but was not found. Soon, however, it was discovered ${ }^{13,14}$ how to construct axion models with arbitrarily large values of $f_{a}$. These were called "invisible" axion models because for $f_{a}>>250 \mathrm{GeV}$, the axion is so weakly coupled that the event rates in the axion search experiments mentioned above are hopelessly small. However it turned out that such axions are still constrained by astrophysical and cosmological considerations.

\section{Cosmological and astrophysical constraints on the axion mass}

Light weakly coupled bosons, such as the arion, are severely constrained by stellar evolution because stars emit such particles from their whole volume, whereas they emit photons which couple more strongly to matter only from their surface. This idea has been very extensively applied to the axion using a variety of stellar objects (the sun, red giants, neutron stars, white dwarfs, and recently the supernova SN1987a) and using a variety of axion producing thermal processes in those objects (the Primakoff process, Comptonlike scattering, axion bremsstrahlung in electron-nucleon and nucleon-nucleon scattering, etc.). ${ }^{15}$ The length of the neutrino pulse from SN1987a rules ${ }^{16}$ out the axion mass range $10^{-3} \mathrm{eV}<m_{a}<$ few eV. The evolution of red giants rules out few $\mathrm{eV}<m_{a}<500 \mathrm{keV}$. The accelerator-based axion searches rule out $m_{a}>10 \mathrm{keV}$. Finally, a recent telescope search for monochromatic photons from the $2 \gamma$ decay of relic axions rules out $3 \mathrm{eV}<m_{a}<8 \mathrm{eV}$. Combining these constraints, one concludes that all axions with mass $m_{a}>10^{-3} \mathrm{eV}$ are ruled out except possibly for a rather unlikely window near $m_{a}=O(\mathrm{eV})$. This window is open only to axions whose coupling to the electron is much suppressed.

Cosmology implies a lower limit on the axion mass of the order of $10^{-6} \mathrm{eV}$. This limit follows from the requirement that the present cosmological axion energy density not exceed the critical density for closing the universe. The first contribution to the cosmological axion energy density is due to axion production caused by the realignment of the vacuum during the QCD phase transition. ${ }^{17}$ There may also be a second contribution due to axions radiated by cosmic arion strings. ${ }^{18,19}$ Let us briefly discuss these two contributions in succession.

At very high temperatures the $U_{P Q}(1)$ symmetry is restored. It becomes spontaneously broken when the temperature drops below a critical temperat ure $T_{P Q}$ of order $f_{a}$. 
At these high temperatures, however, the non-perturbative QCD effects (instantons and the like), which will give the axion its mass and thereby align the vacuum expectation value of $a(x) / f_{a}$ in the CP conserving $\bar{\theta}=0$ direction, are highly suppressed. When they do turn on near the QCD phase transition, the axion field $a(x)$ will start to oscillate about the CP conserving minimum of the effective potential. The energy density stored in these axion field oscillations today equals approximately ${ }^{17}$

$$
\rho_{a}^{\text {vac }}\left(t_{0}\right) \approx \rho_{\text {crit }}\left(t_{0}\right)\left(\frac{0.6 \cdot 10^{-5} \mathrm{eV}}{m_{a}}\right)^{7 / 6}\left(\frac{200 \mathrm{MeV}}{\Lambda_{Q C D}}\right)^{2}\left(\frac{75 \mathrm{~km} / \mathrm{s} \cdot \mathrm{Mpc}}{H_{0}}\right)^{2}
$$

where $\rho_{\text {crit }}\left(t_{0}\right)$ is the present critical energy density for closing the universe, $H_{0}$ is the present value of the Hubble constant and $\Lambda_{Q C D}$ is the QCD scale factor. Equation 8 implies the bound $m_{a} \gtrsim 0.610^{-5} \mathrm{eV}$.

It should be emphasized however that there are many sources of uncertainty in the estimate of the axion cosmological energy density and that Eq. 8 only provides us with a very rough estimate. For example, the axion energy density can be diluted by the entropy release due to the decay of heavy particles which decouple before the QCD epoch but decay afterwards. ${ }^{20}$ It can also be diluted by the entropy release associated with a first order QCD phase transition. On the other hand, if the QCD phase transition is first order, ${ }^{21}$ the sudden change in the axion mass at the critical temperature may increase $\rho_{a}\left(t_{0}\right)$ relative to Eq. 8. Finally, if inflation occurs after the Peccei-Quinn phase transition at which $U_{P Q}(1)$ gets spontaneously broken, then the initial vacuum angle $a / f_{a}$ gets homogenized over the whole of the visible universe and $\rho_{a}\left(t_{0}\right)$ could be accidentally suppressed if the initial angle happens to be very close to the $C P$ conserving $\bar{\theta}=0$ direction. The extent to which it can be suppressed depends upon the inflationary model. ${ }^{22}$

If there is no inflation after the phase transition during which the $U_{P Q}(1)$ symmetry becomes spontaneously broken, cosmic axion strings also make a contribution to the cosmological axion energy density. However, there has been a controversy in the literature over the size of this contribution. One view ${ }^{18}$ is that it is about a factor 100 times larger than the contribution from the realignment of the vacuum, discussed above, and hence that it implies a constraint on the axion mass about a 100 times more severe: $m_{a} \gtrsim 10^{-3}$ $\mathrm{eV}$ instead of $m_{a} \gtrsim 10^{-5} \mathrm{eV}$. The second view ${ }^{19}$ is that the contributions from vacuum realignment and from cosmic axion strings are of the same order of magnitude. Computer simulations in Ref. 19 support this second estimate. 
D. The axion as a dark matter candidate

The $10^{-6} \mathrm{eV} \leqslant m_{a}<10^{-3} \mathrm{eV}$ mass range is the only one still allowed for the axion. All masses larger than $10^{-3} \mathrm{eV}$ have been ruled out either by previous searches, stellar evolution constraints or SN1987a. Axion masses smaller than approximately $10^{-6} \mathrm{eV}$ require that there be the right kind of inflation after the Peccei-Quinn phase transition and that the initial vacuum misalignment angle happens to be small.

Cosmic axions are "cold dark matter" because they are non-relativistic from the moment they acquire their mass during the QCD phase transition. ${ }^{17,23}$ Cold dark matter with a flat or nearly flat spectrum of primordial density perturbations and some "biasing" in the extent to which light traces matter continues to be, it the post-Cobe era, an attractive and apparently viable scenario of galary formation. Thus the dark matter which appears clustered in halos around galaxies may be axions with mass in the $10^{-5} \mathrm{eV}$ to $10^{-6} \mathrm{eV}$ range. If the halo of our own galaxy is made up exclusively of axions, their density in the solar neighborhood ${ }^{24}$ would be of order $0.5 \cdot 10^{-24} \mathrm{gr} / \mathrm{cm}^{3}=0.3 \mathrm{GeV} / \mathrm{cm}^{3}$ and their velocity dispersion would be $10^{-3}$ times the speed of light. The possibility of detecting these dark matter axions constitutes an exciting prospect and one which, as we will see, is very real. If a signal is found in the proposed experiment, the long standing question why the strong interactions conserve $P$ and $C P$ will finally be answered, but also a new window on our universe will have opened up. In recent papers, ${ }^{25,26}$ it has been shown that a cosmic axion signal would be a very rich source of information about our galaxy.

Figure 1, below, summarizes the present laboratory, astrophysical and cosmological limits on the axion mass. (From Raffelt ${ }^{15}$ ).

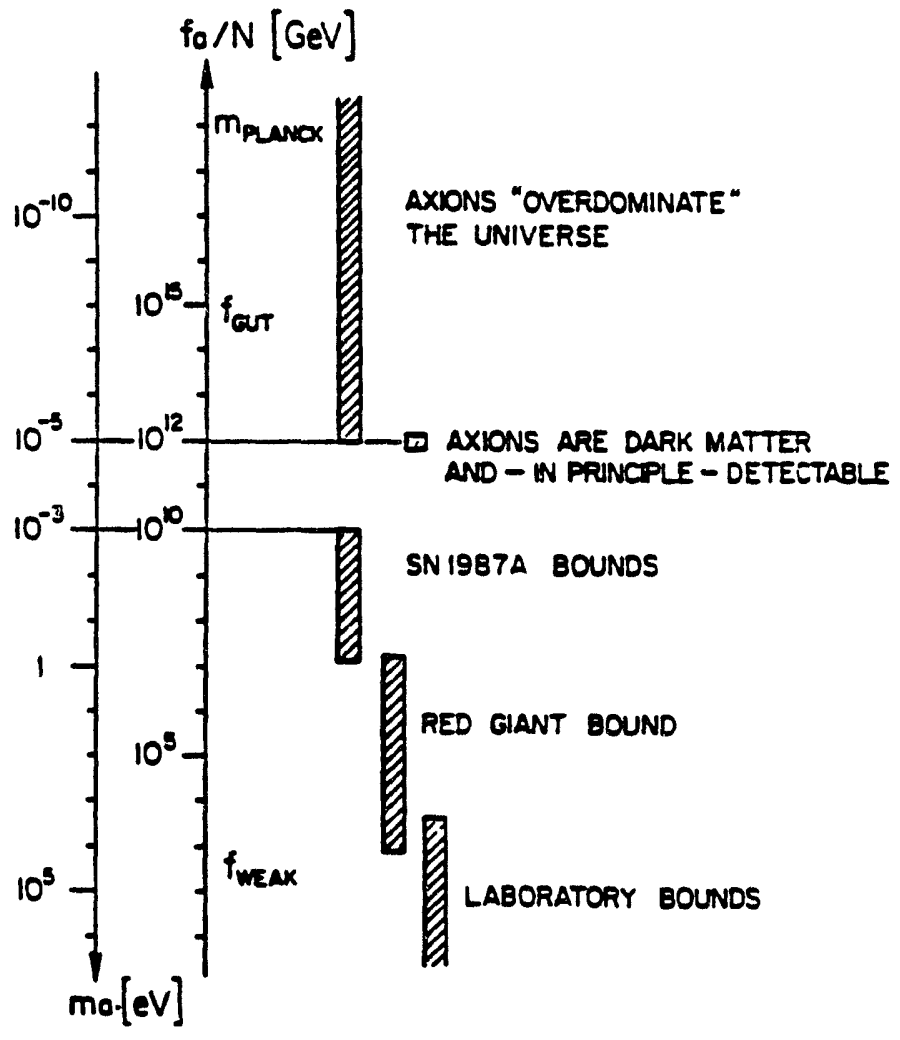




\section{THE CAVITY DETECTOR \\ OF GALACTIC HALO AXIONS}

\section{A. The detector principle}

About ten years ago it was pointed out that axions can be searched for by stimulating their conversion to photons in a strong magnetic field. ${ }^{27}$ The relevant coupling is given in Eq. 6. In particular, an electromagnetic cavity permeated by a strong magnetic field can be used to detect galactic halo axions. The latter have velocities $\beta$ of order $10^{-3}$ and hence their energies

$$
\epsilon_{a}=m_{a}+\frac{1}{2} m_{a} \beta^{2}
$$

have a spread of order $10^{-6}$ above the axion mass. Consider a cylindrical electromagnetic cavity of arbitrary cross-sectional shape, permeated by a large static longitudinal magnetic field $\vec{B}_{0}$. When the frequency $\omega=2 \pi f$ of an appropriate cavity mode equals $m_{a}[1+$ $O\left(10^{-6}\right)$ ], galactic halo axions can convert to quanta of excitation (photons) of that cavity mode. Only the $\mathrm{TM}_{\text {nlo }}$ modes couple in the limit where the cavity is much smaller than the de Broglie wavelength $\lambda_{a}=2 \pi\left(\beta m_{a}\right)^{-1} \approx 2 \pi 10^{3} m_{a}^{-1}$ of the galactic halo axions. The power on resonance from axion $\rightarrow$ photon conversion into the $\mathrm{TM}_{n \ell 0}$ mode is ${ }^{27,28}$

$$
\begin{aligned}
P_{n \ell}= & \left(\frac{\alpha}{\pi} g_{\gamma} \frac{1}{f_{a}}\right)^{2} V B_{0}^{2} \rho_{a} C_{n \ell} \frac{1}{m_{a}} \operatorname{Min}\left(Q_{L}, Q_{a}\right) \\
= & 3 \cdot 10^{-26} \mathrm{Watt}\left(\frac{V}{3 \mathrm{~m}^{3}}\right)\left(\frac{B_{0}}{7 \text { Tesla }}\right)^{2} C_{n \ell}\left(\frac{g_{\gamma}}{0.36}\right)^{2} \\
& \cdot\left(\frac{\rho_{a}}{\frac{1}{2} \cdot 10^{-24} \mathrm{gr} / \mathrm{cm}^{3}}\right)\left(\frac{m_{a}}{2 \pi(1 \mathrm{GHz})}\right) \min \left(Q_{L}, Q_{a}\right)
\end{aligned}
$$

where $V$ is the volume of the cavity, $B_{0}$ is the effective static magnetic field strength, $C_{n}$ c is a mode-dependent form factor, $\rho_{a}$ is the density of galactic halo axions at the earth, $Q_{L}$ is the loaded quality factor of the cavity and $Q_{a} \simeq 10^{6}$ is the "quality factor" of the galactic halo axions, i.e., the ratio of their energy to their energy spread.

The product $B_{0}^{2} C_{n \ell}$ is defined as follows. For $T M_{n \ell 0}$ modes, one has

$$
\begin{aligned}
& \vec{E}_{\omega}=\hat{z} \psi_{n \ell}(x, y) e^{-i \omega_{n \ell} t} \\
& \vec{B}_{\omega}=\frac{i}{\omega_{n \ell}} \hat{z} \times \vec{\nabla}_{t} \psi_{n \ell}(x, y) e^{-i \omega_{n \ell} t}
\end{aligned}
$$

where $\mathrm{x}$ and $\mathrm{y}$ are the transverse coordinates and $\psi_{n \ell}$ satisfies

$$
\left(\frac{\partial^{2}}{\partial \mathrm{x}^{2}}+\frac{\partial^{2}}{\partial \mathrm{y}^{2}}\right) \psi_{n \ell}+\omega_{n \ell}^{2} \epsilon \psi_{n \ell}=0
$$

and the condition 


$$
\psi_{n \ell} \mid r=0
$$

on the metallic boundaries $\Gamma$ of the cross-sectional area $S$ of the cavity. $\epsilon$ is the dielectric constant which may depend upon $x$ and $y$. The magnetic permeability $\mu$ was assumed to be equal to one everywhere. The product $B_{0}^{2} C_{n \ell}$ is defined by

$$
B_{0}^{2} C_{n \ell}=\frac{\left|\int_{V} d^{3} x \psi_{n \ell}(x, y) \vec{B}_{0}(\vec{x}) \cdot \hat{z}\right|^{2}}{V \int_{V} d^{3} x\left|\psi_{n \ell}(x, y)\right|^{2} \epsilon(x, y)}
$$

To get an idea of the mode number dependence of $B_{0}^{2} C_{n \ell}$ consider the simple case where $\vec{B}_{0}(\vec{x})$ is homogeneous and longitudinal $\vec{B}_{0}(\vec{x})=B_{0} \hat{z}$. Then, for a cavity of rectangular cross-section, one has

$$
C_{n \ell}=\left\{\begin{array}{cl}
\frac{64}{\pi^{4} n^{2} \ell^{2}} & \text { for } n \text { and } \ell \text { odd } \\
0 & \text { otherwise }
\end{array}\right.
$$

For a cavity of circular cross-section

$$
C_{n m}=\frac{4}{\left(x_{0 n}\right)^{2}} \delta_{n m}
$$

where $x_{0 n}$ is the $\mathrm{n}^{\text {th }}$ zero of the Bessel function $J_{0}(x)$. One sees that $C_{n \ell}$ is a sharply decreasing function of mode-number. If at all possible, one should use the fundamental TM mode. This is what we intend to do. From here on, we will suppress the indices $n, l$.

B. The sensitivity as a function of search rate

To detect the power $P$, a hole must be made in the cavity wall through which the electromagnetic radiation can be brought to the front end of a microwave receiver. The quality factor $Q_{L}$ which appears in Eq. 10 is the loaded quality factor given by

$$
\frac{1}{Q_{L}}=\frac{1}{Q_{w}}+\frac{1}{Q_{h}}
$$

where $\frac{1}{Q_{w}}$ is the contribution due to absorption into the cavity walls and $\frac{1}{Q_{h}}$ is the contribution from the hole. The maximum power that can be brought to the front end of the microwave receiver is $\frac{Q_{L}}{Q_{h}} P$ where $P$ is given by Eq. 10 .

Because the axion mass is only known in order of magnitude at best, the cavity must be tunable and a large range of frequencies must be explored seeking the axion signal. The cavity can be tuned by inserting a dielectric rod into it, ${ }^{29}$ or by moving a dielectric 
rod or metal post sideways inside the cavity. ${ }^{30,31}$ Using Eq. 10, one finds that to obtain a given signal to noise ratio $s / n$, the search rate is

$$
\begin{aligned}
\frac{d f}{d t}= & \frac{55 \mathrm{GHz}}{\text { year }}\left(\frac{4}{s / n}\right)^{2}\left(\frac{V}{3 \mathrm{~m}^{3}}\right)^{2}\left(\frac{B_{0}}{7 \text { Tesla }}\right)^{4} C^{2}\left(\frac{g_{\gamma}}{0.36}\right)^{4} \\
& \cdot\left(\frac{\rho_{a}}{\frac{1}{2} \cdot 10^{-24} \mathrm{gr} / \mathrm{cm}^{3}}\right)^{2}\left(\frac{5 \mathrm{~K}}{T_{n}}\right)^{2}\left(\frac{f}{1 \mathrm{GHz}}\right)^{2} \cdot\left\{\begin{array}{ccc}
Q_{w} / Q_{a} & \text { if } Q_{w}<3 Q_{a} \\
\frac{27}{4}\left(1-\frac{Q_{a}}{Q_{w}}\right)^{2} & \text { if } & Q_{w}>3 Q_{a}
\end{array}\right.
\end{aligned}
$$

where $T_{n}$ is the sum of the physical temperature of the cavity plus the system noise temperature of the microwave receiver, and where $Q_{a} \equiv 10^{6}$ now. Equation 17 was derived assuming

1) that when $Q_{L}<Q_{a}$, i.e., when the cavity bandwidth is larger than the axion bandwidth, one uses the possibility of looking at $Q_{a} / Q_{L}$ axion bandwidths simultaneously, $^{32}$

2) that $Q_{h}$ has been adjusted so as to maximize the search rate. For $Q_{w}<3 Q_{a}$, the optimal $Q_{h}=\frac{1}{2} Q_{w}$ (and hence $Q_{L}=\frac{1}{3} Q_{w}$ ) whereas for $Q_{w}>3 Q_{a}$, the optimal $Q_{h}$ occurs at $Q_{L}=Q_{a}$.

The best possible quality factors attainable at present, using oxygen-free copper, are of the order of

$$
Q_{w} \simeq \frac{R}{\delta} \simeq 1.3 \cdot 10^{5}\left(\frac{\mathrm{GHz}}{f}\right)^{\frac{2}{3}}
$$

where $R$ is some measure of the transverse size of the cavity (or of the cavity cells if the cavity is divided into cells; see below) and $\delta$ is the anomalous skin depth. One has $R \sim f^{-1}$ and $\delta \sim f^{-\frac{1}{3}}$. Note that on a logarithmic frequency scale the search rate to achieve a given signal to noise ratio is not sharply frequency dependent if $T_{n}$ and $C$ are not sharply frequency dependent. Indeed, by combining Eqs. 17 and 18, one finds

$$
\frac{d \ln f}{d t} \sim\left(\frac{C(f)}{T_{n}(f)}\right)^{2} f^{\frac{1}{3}} .
$$

Eq. 17 shows that a cosmic axion search sensitive to the local galactic halo density is feasible with presently available technology provided a sufficiently large magnet is available and provided the cavity can be designed to keep $C=O(1)$ at all frequencies of interest. 


\section{The optimization of cavity design}

The problem of cavity design is the following. A large volume empty cylindrical cavity has a low resonant frequency in its lowest TM mode: $f=0.115 \mathrm{GHz}\left(\frac{1 \mathrm{~m}}{R}\right)$ where $R$ is the radius of the cavity. In terms of the physical (not angular) frequency, $1 \mathrm{GHz}$ corresponds to $4.135 \mu \mathrm{eV}$, etc. Thus a large cylindrical cavity is adequate for searching the low end of the axion mass range of interest. The problem is: how does one search the higher frequencies? This question was addressed in a paper by C. Hagmann et al. ${ }^{30}$ which also discussed various cavity tuning schemes and presented the results of computer simulations to optimize cavity design. It was found in Ref. 30 that the best way to tune a single cylindrical cavity is by translating a dielectric rod or a metal post sideways inside the cavity. This method has been successfully tested in the pilot cosmic axion detector at the University of Florida, and it is the method we plan to use in the experiment proposed here. Secondly, Ref. 30 concluded that the best way to extend the search to high frequencies is the straightforward approach of power-combining many identical cavities which fill up the volume inside the magnet bore. This method entirely avoids the problems of mode localization and resonance crowding which plague the other approaches considered in Ref. 30. It allows one to maintain $C \simeq O(1)$ at all frequencies, albeit at the cost of increasing engineering complexity when the frequency is increased. This method was tested at room temperature and found to work entirely satisfactorily. ${ }^{33}$ 


\section{SUMMARY OF PREVIOUS CAVITY SEARCHES}

At the present time two groups have conducted axion searches using cavity detectors. During 1986-1989 a collaboration between Rochester, Brookhaven, and Fermilab (RBF) carried out a search which covered energies in the 4.5-18 $\mu \mathrm{eV}(1-4 \mathrm{GHz})$ range. ${ }^{29,34,35}$ In 1989-90 the Florida (UF) group scanned 5.5-7 $\mu \mathrm{eV}(1.3-1.6 \mathrm{GHz}){ }^{31}$ These experiments are discussed in the following sections.

These pilot efforts were valuable in validating the concept of the cavity microwave technique. Nevertheless, in view of the fact that these experiments fell short of the required power sensitivity by $\sim 1000$, it must be admitted that the limits they set are hardly stringent, and in sum they have had minimal impact on the scientific issue of axions, particle physics, and cosmology.

\section{A. The RBF experiment}

The first axion search was carried out by the RBF group. ${ }^{29,34,35}$ They used a $5.8 \mathrm{~T}$ magnet (7.4 T above $1.4 \mathrm{GHz}$ ) and a cavity volume ranging from $1 \times 10^{-2} \mathrm{~m}^{3}$ at the low frequency end of their range to $1 \times 10^{-3} \mathrm{~m}^{3}$ at the high frequency end, giving

$$
0.36 \mathrm{~T}^{2} \mathrm{~m}^{3}>B_{0}^{2} V>0.06 \mathrm{~T}^{2} \mathrm{~m}^{3}
$$

over the frequency range scanned.

Each cavity was tuned by insertion of a dielectric rod along the cavity axis. A total of 7 cavities was employed to cover the $1-4 \mathrm{GHz}$ range. At the highest frequencies, the cavity had a high aspect ratio and was substantially smaller than the magnet bore. The cavity emission was amplified by a cooled GaAs FET amplifier $\left(T_{n} \approx 10 \mathrm{~K}\right)$, mixed down to audio frequencies, and spectrum analyzed using a 64 -channel bandpass-filter bank. The power in each channel was recorded and then searched for peaks off-line. All peaks of $5 \sigma$ in one bin or $4 \sigma$ in two adjacent bins were checked afterwards with and without the magnetic field on. No candidate peak survived.

The results of this experiment are shown in Fig. 2. The shaded region is the range of $g_{a \gamma \gamma}$ that the RBF collaboration excluded. Here, $g_{a \gamma \gamma} \equiv g_{\gamma} \alpha / \pi f_{a}$, with $g_{\gamma}$ and $f_{a}$ defined in Eqs. 4-7. The noise temperature of the amplifier was $\sim 10 \mathrm{~K}$ and the physical temperature was $4 \mathrm{~K}$, making the sensitivity of the receiver approximately a factor of 2000 short of the DFSZ limit in the mass range scanned. (In view of the problems of mode localization found in later analyses ${ }^{30}$ of low-aspect-ratio cavities with axial tuning rods, it is likely that the limit at the high mass end is substantially weaker than shown in Fig. 2.) 


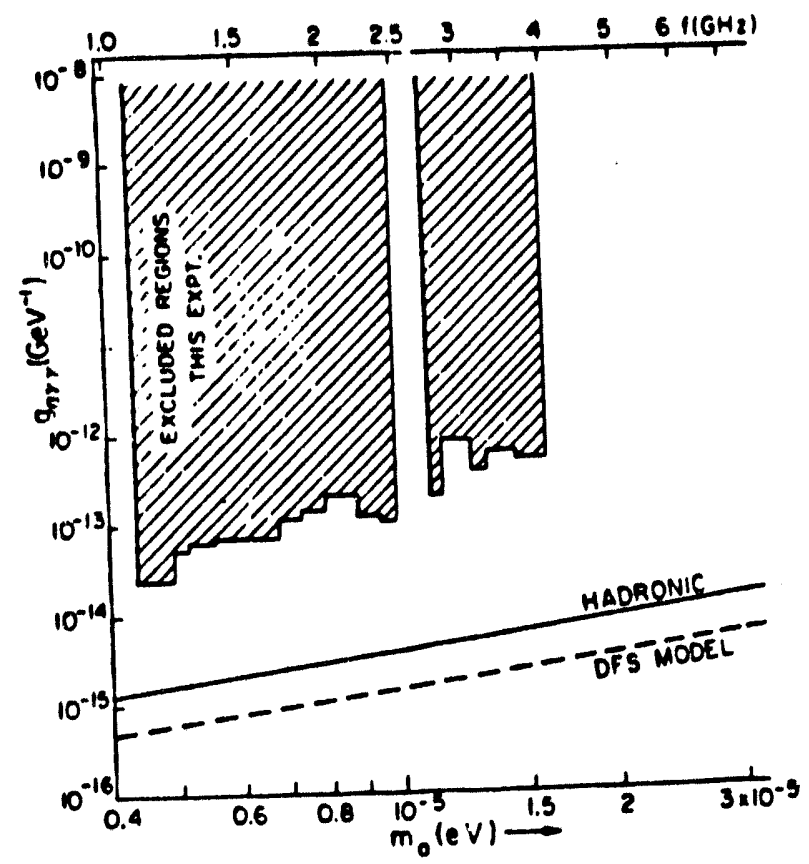

Fig. 2. Results from the RBF axion search. The shaded area shows the values of gary excluded as a function of axion mass, assuming that the local axion density equals the halo density. (From Ref. 35.)

\section{B. The UF experiment}

The UF detector began taking data in 1989. ${ }^{31,36}$ A sketch is shown in Fig. 3. This system has been operated as a "pilot" experiment to test axion detection concepts. The various components and their performance will be described in some detail.

\section{Magnet}

The detector uses a superconducting magnet $40 \mathrm{~cm}$ long with $17 \mathrm{~cm}$ inner bore. The central field is $8.6 \mathrm{~T}$ while the volume-averaged field over the $0.008 \mathrm{~m}^{3}$ volume of the cavity is $7.5 \mathrm{~T}$. This gives $B_{0}^{2} V=0.45 \mathrm{~T}^{2} \mathrm{~m}^{3}$. The magnet is operated in persistent mode, typically at $2.2 \mathrm{~K}$. Temperatures below the $4.2 \mathrm{~K}$ normal boiling point of liquid helium were achieved with a " $\lambda$-fridge," in which a small stainless-steel tube containing a heat exchanger and flow impedance extends well below the surface of the helium. When the upper end of the tube is evacuated, the helium in the tube coois to approrimately $2 \mathrm{~K}$ and in turn lowers the temperature in the outside bath. With this arrangement the helium around the magnet, cavity, and amplifier can be reduced in temperature while the free surface above remains at $4.2 \mathrm{~K}$, reducing superfluid film-flow losses and permitting easy retransfer of the helium without warming up the magnet.

\section{Cavities}

Two cavities were used, differing by $10 \%$ in diameter. The cavities, made from OFHC copper and electropolished to provide good surfaces, were operated in the $\mathrm{TM}_{010}$ mode. 


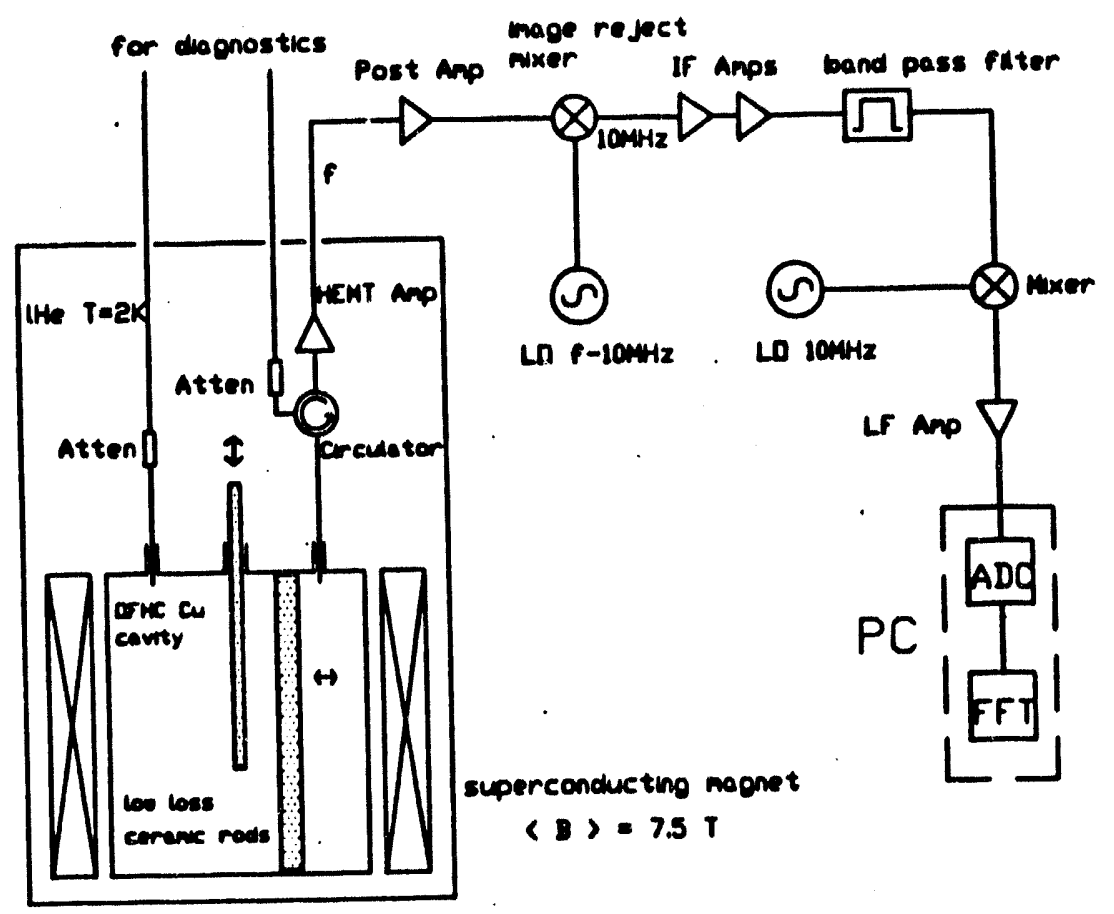

Fig. 3. Block diagram of UF arion detector, microwave circuitry, and data acquisition system.

Figure 4 shows the cavity along with tuning rods and coupling ports. Unloaded quality factors were measured to be $Q_{w} \approx 150,000$, and nearly $T$-independent below $20 \mathrm{~K}$. The wall quality factor for the $T M_{010}$ mode is

$$
Q_{w}=\frac{L}{L+R} \frac{R}{\delta}
$$

where $L$ is the length and $R$ the radius of the cavity; the skin depth $\delta$ in the extreme anomalous skin effect regime is $\delta=\left(\sqrt{3} c^{2} m_{e} v_{F} / 8 \pi^{2} \omega N_{e} e^{2}\right)^{1 / 3} \approx 0.28 \mu \mathrm{m}$. Here, $m_{e}$ is the electron mass, $v_{F}$ the Fermi velocity, and $N_{e}$ the conduction electron density. For the parameters of the cavities used, Eq. 20 predicts $Q_{w}^{A S E}=250,000$, about $60 \%$ larger than what was measured. The discrepancy may be due to parasitic losses at the coupling port, to a small build-up of oxide, and to scratches and deformation of the cavity walls. In the following, we will base our estimates of sensitivity and running times on the experimental $Q$-factors demonstrated in this pilot project. As we shall see, the cavity performance is more than adequate for the proposed experiment.

The larger of the two coupling ports-the major port-is overcoupled and connected to the amplifier. It consists of an inductive loop at the end of a coarial cable. Springloaded $\mathrm{BeCu}$ fingers make electrical connection between the outer sheath of the coax and the cavity, allowing the coupling strength of the cavity to be varied by either changing the insertion depth of the loop or by rotating the loop. We found that either coupling method worked well, allowing $Q_{h}$ to be varied from $2 \times$ overcoupled to substantially undercoupled without affecting other cavity parameters. The smaller coupling port in Fig. 4 is used for 


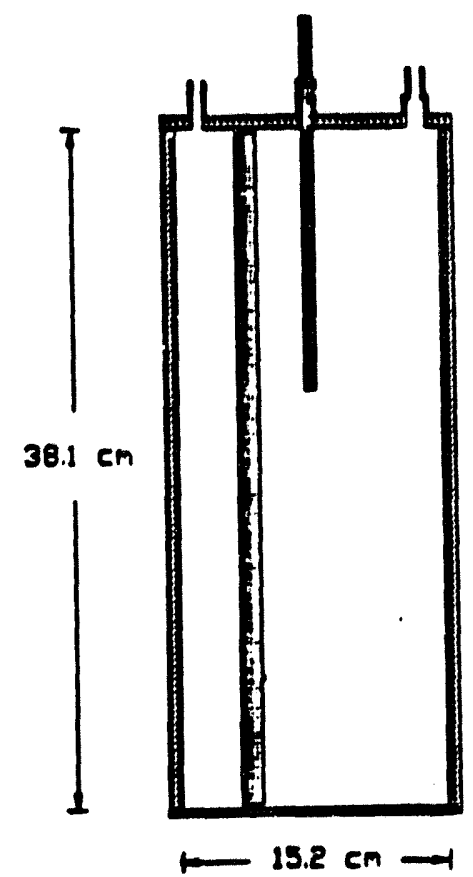

Fig. 4. Cavity with two dielectric tuning rods. The larger rod is translated sideways; the smaller one along the cylinder axis. Two coupling ports are also ahown.

measuring the resonance frequency and quality factor in transmission mode, as well as for other diagnostic measurements.

The cavity is tuned by moving dielectric or metal rods located in the cavity. Two tuning rods are employed: coarse tuning is achieved with a larger rod-either dielectric or metal-that is moved radially while fine tuning uses a small dielectric rod inserted along the cavity aris. The purpose of using two tuning rods is to enable rather large tuning ranges $( \pm 20 \%)$ while avoiding problems of longitudinal mode localization, ${ }^{30}$ which degrades the cavity form factor $C$.

Figs. 5 and 6 show the electric field patterns calculated for the cases of dielectric and metal rods displaced sideways from the cylinder axis. The electric field is enhanced inside the dielectric and is zero inside the metal. The ratios $r / R$ of the tuning rod radii to the cavity radius were chosen small enough to avoid transverse mode localization. ${ }^{30}$ If the tuning rods are enlarged the tuning range is increased but the stored energy becomes more and more localized-either inside the dielectric rod or in the region away from the metal rod.

In the UF experiment, the large rod is manually positioned to set the initial frequency at the beginning of each data acquisition cycle. The small rod is cantilevered from a dovetail translation slide and moved by a stepper motor under control of the data acquisition system.

Both dielectric tuning rods are made from di-magnesium-titanate/magnesium- 


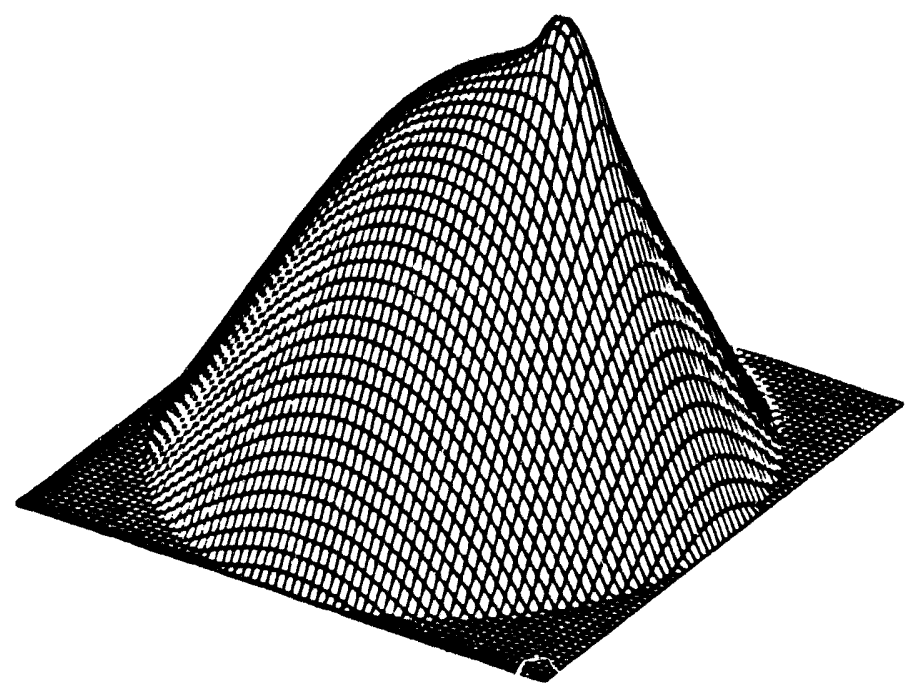

Fig. 5. Electric field for the TMO10 mode of a cylindrical cavity with a radially displaced dielectric rod. The sod, with $e=9.5$, has a diameter equal to $9.3 \%$ of the cavity diameter and is displaced halfway between the center and wall of the cavity (where the little bump in the field profile is).

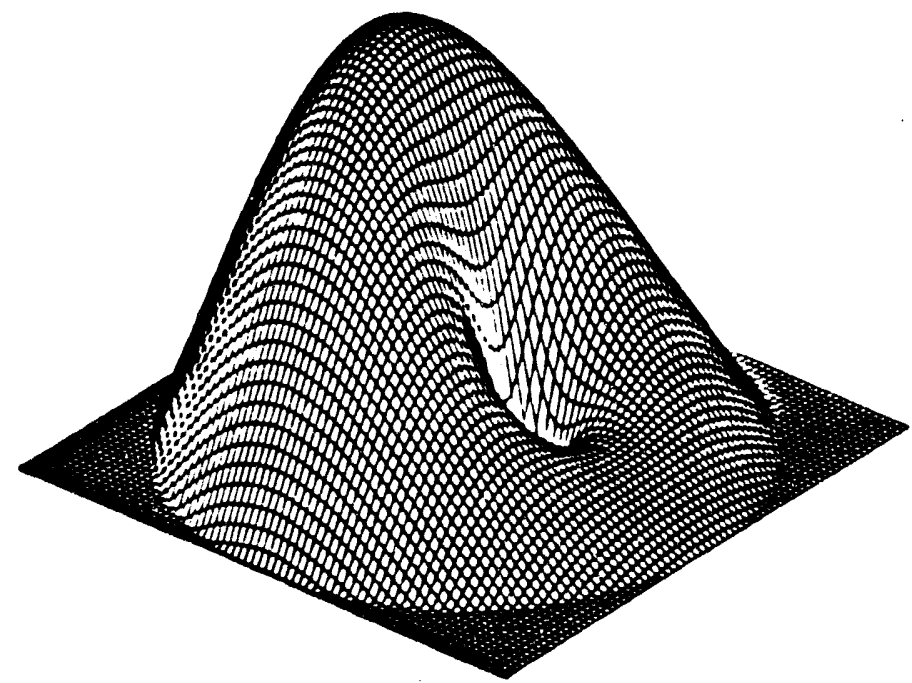

Fig. 6. Electric field for the $T M_{010}$ mode of a cylindrical carity with a radially displaced metal rod. The rod has a diameter equal to $8.2 \%$ of the cavity diameter and is displaced a quarter of the way between the center and wall of the cavity (where the sero in the field profile is).

aluminate (SMAT-9.5). ${ }^{37}$ This material has $\epsilon \approx 9.5$ and $\tan \delta \leq 10^{-5}$. The metal tuning rod is copper. Measurements of a cavity with a metal tuning rod show a decrease in $Q_{w}$ from 150,000 to 50,000 . This is partially mitigated by an increase in the form factor from 0.5 to 0.6 , making the product $C^{2} Q$ about a factor of 2 worse for metal than dielectric tuning materials.

As the cavity is tuned there are a number of mode crossings between the $T M_{010}$ 
mode and various TE modes. Because of unavoidable mixing of the two modes in this experiment there is a region a few hundred $\mathrm{kHz}$ wide at each crossing where the detector does not work. These holes comprise less than $0.2 \%$ of the scanned spectrum. (These frequencies will be scanned in the proposed experiment by adjusting a second tuning rod.) Figure 7 shows one such crossing in Cavity I. This figure, which shows the resonant frequency as a function of the insertion depth of the small tuning rod, also illustrates the precision of cavity tuning. A travel of $1 \mathrm{~cm}$ leads to a $1 \mathrm{MHz}$ change in the $T M_{010}$ frequency. The smallest step is $\approx 4 \mu \mathrm{m}$, which changes the frequency by about $400 \mathrm{~Hz}$.

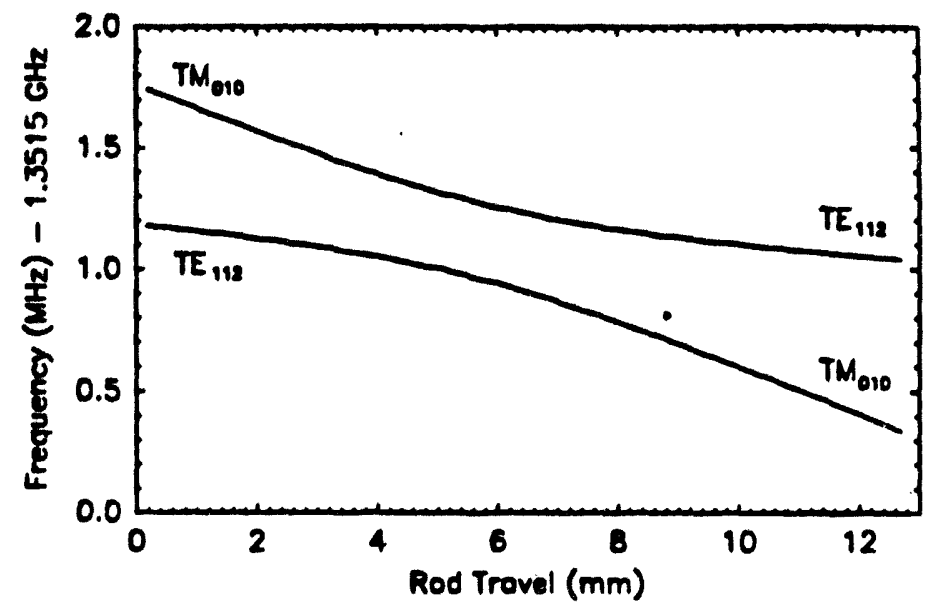

Fig. 7. Measured mode crossing between the $T E_{112}$ and $T M_{010}$ cavity modes. The ordinate is the insertion depth of the small tuning rod along the cavity axis.

\section{Microwave system}

The cryogenic microwave amplifiers were purchased from Berkshire Technologies. ${ }^{38}$ These are three-stage GaAs amplifiers, with the first stage device being a high electron mobility transistor (HEMT). The amplifiers work at all temperatures between $300 \mathrm{~K}$ and $2 \mathrm{~K}$, providing about $35 \mathrm{~dB}$ power gain, with substantially improved noise performance at the lowest temperature.

Figs. 8 and 9 show noise temperature as a function of frequency for two amplifiers. The physical temperature of the amplifiers was $4.2 \mathrm{~K}$. These data were obtained using a variable temperature technique. ${ }^{39}$ The minimum $T_{n}$ was $3 \mathrm{~K}$ and the typical value over the band used was $T_{n} \approx 4 \mathrm{~K}$. (Note that these values include approximately $0.2 \mathrm{~K}$ contribution from the following amplifier, which operated at $300 \mathrm{~K}$.) The noise temperature is $T$. independent below $20 \mathrm{~K}$; above $20 \mathrm{~K}$ it increases gradually, reaching $T_{n} \approx 100 \mathrm{~K}$ when the amplifier is at $300 \mathrm{~K}$.

Magnetically shielded cryogenic circulators ${ }^{40}$ are inserted between the cavity and amplifier input terminal to permit the cavity to be isolated from the amplifier. The third arm of the circulator is then used to bring microwave power to the major port, so that 


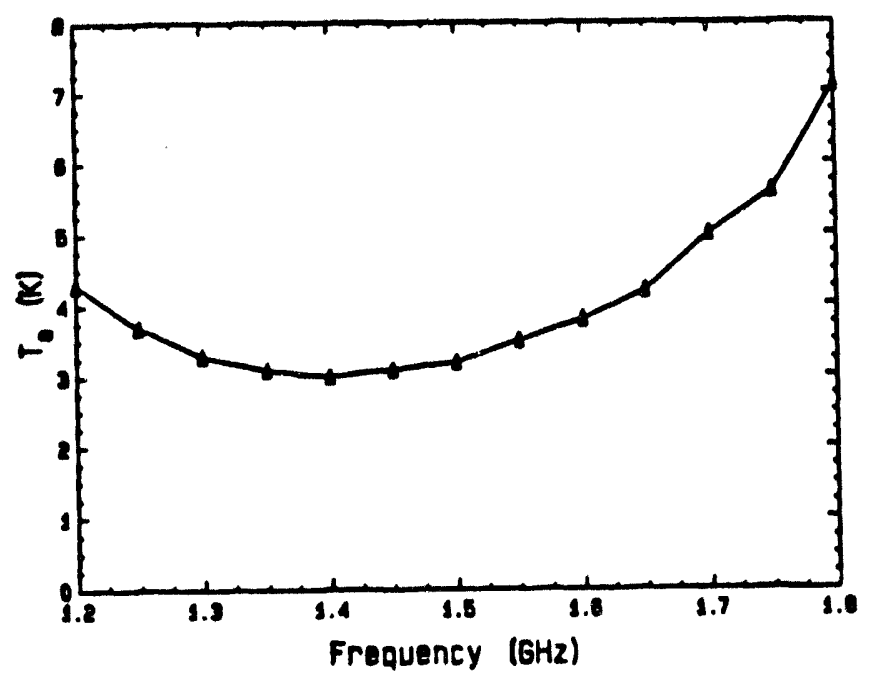

Fig. 8. Noise temperature of Berkshire L-1.5-30H amplifier at a physical temperature of $4.2 \mathrm{~K}$. The amplifier has a midband gain of $34 \mathrm{~dB}$.

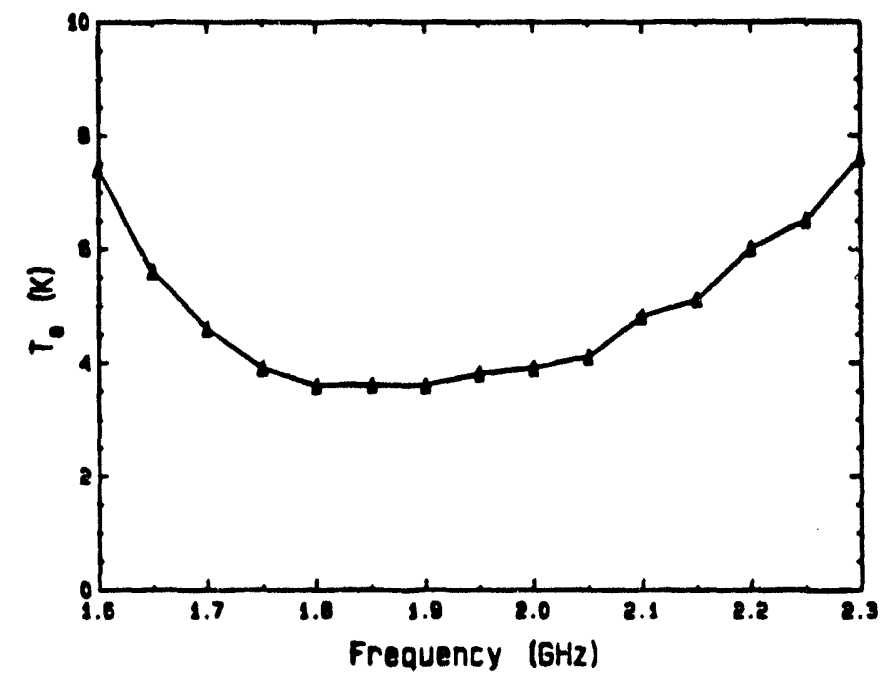

Fig. 9. Noise temperature of Berkshire S-2.35-30HR amplifier at a physical temperature of $4.2 \mathrm{~K}$.

The amplifier has a midband gain of $35 \mathrm{~dB}$.

its reflection coefficient can be measured and coupling adjusted. A $50 \mathrm{~dB}$ attenuator in the helium bath kept room temperature noise from entering the cavity.

The remainder of the experimental setup in Fig. 3 is the double-superheterodyne receiver, the digital spectrum analyzer, and the computer control system. The output of the cryogenic amplifier is further amplified ( $50 \mathrm{~dB}$ ) by a $300 \mathrm{~K}$ microwave amplifier and converted to the $10.7 \mathrm{MHz}$ first IF frequency by an image reject mixer. This mirer, assembled from commercially available components, used a synthesizer as local oscillator. The rejection of the unwanted sideband was $\geq 18 \mathrm{~dB}$ and averaged $22 \mathrm{~dB}$.

The IF amplifiers have a combined gain of $60 \mathrm{~dB}$; they are followed by an 8-pole crystal 
filter, which determines the bandwidth of the system. The filter response is reasonably flat over its $30 \mathrm{kHz}$ passband, with over $50 \mathrm{db}$ rejection of out-of-band signals. This filter has a significant temperature variation to its response. It was therefore necessary to enclose it in a temperature-stabilized enclosure, which reduced drift to an acceptable level. The second converter uses a double-balanced mixer and $10.67 \mathrm{MHz}$ local oscillator. This provides an audio signal spanning $0-30 \mathrm{kHz}$ which undergoes a final amplification before $A / D$ conversion.

\section{Spectrum analyzer}

We use a digital fast Fourier transform (FFT) scheme to spectrum analyze the signal from the cavity. The analyzer consists of a 16 bit A/D converter coupled with a digital signal processor. The audio signal (bandwidth $30 \mathrm{kHz}$ ) is sampled at a $70 \mathrm{kHz}$ rate, with a total of 64 samples measured. These data are Fourier transformed and the power spectrum calculated, giving a resolution of $\approx 1 \mathrm{kHz}$, the width of the axion signal when $m_{a}=4.135 \mu \mathrm{eV}$, or $f=1 \mathrm{GHz}$. Successive spectra are averaged to obtain the power spectrum emitted from the cavity over its bandwidth.

The signal processing board $\mathrm{d}^{41}$ consists of the $\mathrm{A} / \mathrm{D}$ converter, a $40 \mathrm{MHz}$ signal processing chip, ${ }^{42}$ and $64 \mathrm{~kb}$ of memory, all located in the 80386-based PC which serves as the system controller. The board is fast enough to perform the FFT, calculate the power spectrum, and make the average in just under $0.6 \mathrm{~ms}$. While one set of samples is being taken, the previous one is Fourier analyzed and averaged, so that the analyzer has no dead time.

The time for a single A/D scan is $0.9 \mathrm{~ms}$; a total of $10^{5}$ spectra are acquired and averaged in $90 \mathrm{sec}$. Immediately afterwards, each average spectrum is searched for $2 \sigma$ peaks in single bins and combination of neighboring bins. If a peak is found, another set of $10^{5}$ spectra is taken and averaged with the first. If the peak remains statistically significant this process is repeated up to a maximum of 5 times, after which the peak is flagged for later investigation. This repetition improves the signal/noise rativ of candidate signals by just over a factor of two.

To continue the run, the computer writes the completed spectrum to disk, steps the small tuning rod, introduces a frequency-swept test signal into the cavity minor port, measures the cavity $Q$ and resonant frequency, and instructs the signal processing board to get the next set of spectra.

Figure 10 shows a typical spectrum, with no peaks exceeding the $2 \sigma$ level. Figure 11 shows one of several peaks which survived the test described above. When re-examined with the magnetic field $B_{0}$ off, all of these peaks persisted, and therefore were not signals coming from axion conversion. Instead they were from external sources, in particular from the computer and other digital equipment in the vicinity of the experiment. 


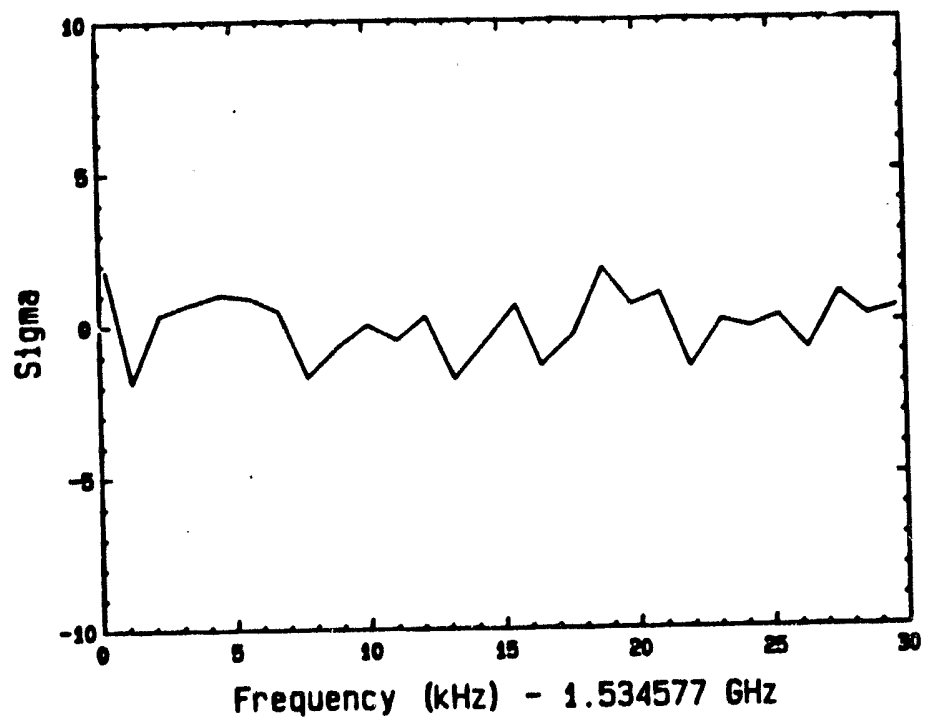

Fig. 10. Typical power spectrum.

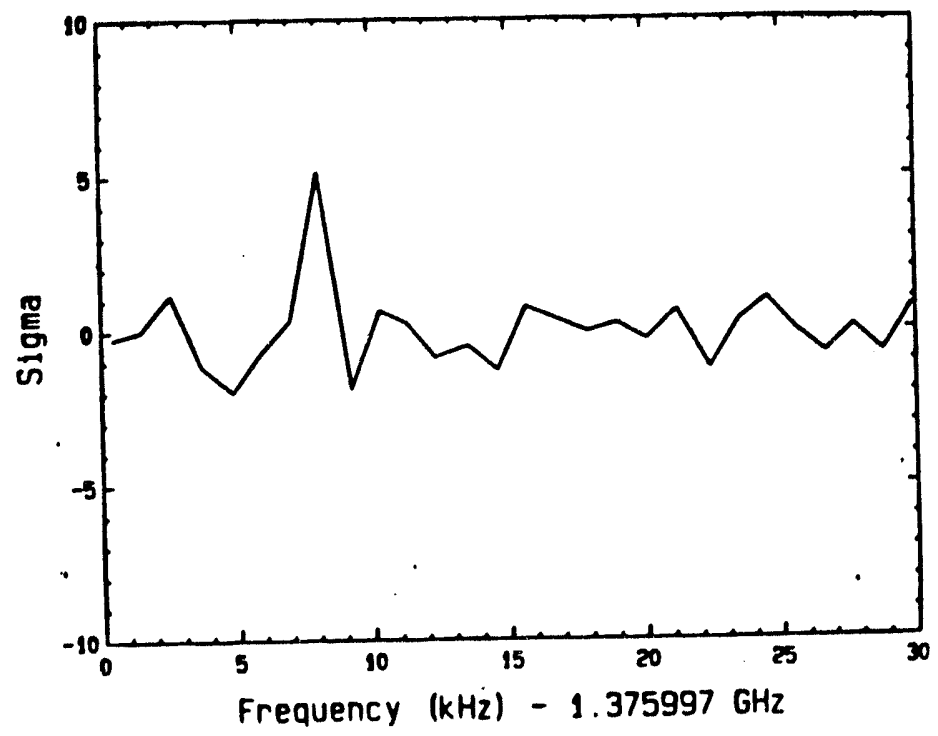

Fig. 11. Power spectrum with candidate peak.

\section{Results}

During Fall and Winter of 1989-90 the detector was operated for a total of five months. Two separate runs were made over $1.32-1.44 \mathrm{GHz}$, one at bath temperature $T=2.2 \mathrm{~K}$ and the other at $T=4.2 \mathrm{~K}$, and their statistics combined. A single run was made over $1.44-1.60 \mathrm{GHz}$ at $2.2 \mathrm{~K}$. The experiment ${ }^{43}$ is thus able to set the upper limit on $g_{a \gamma \gamma}^{2}$ (for $\left.\rho_{a}=\rho_{\text {halo }}\right)$ plotted in Fig. 12. Also shown is the limit obtained by the RBF group. ${ }^{29,34,35}$

The sensitivity of the detector is set by thermal noise. The noise power fluctuation is given by 


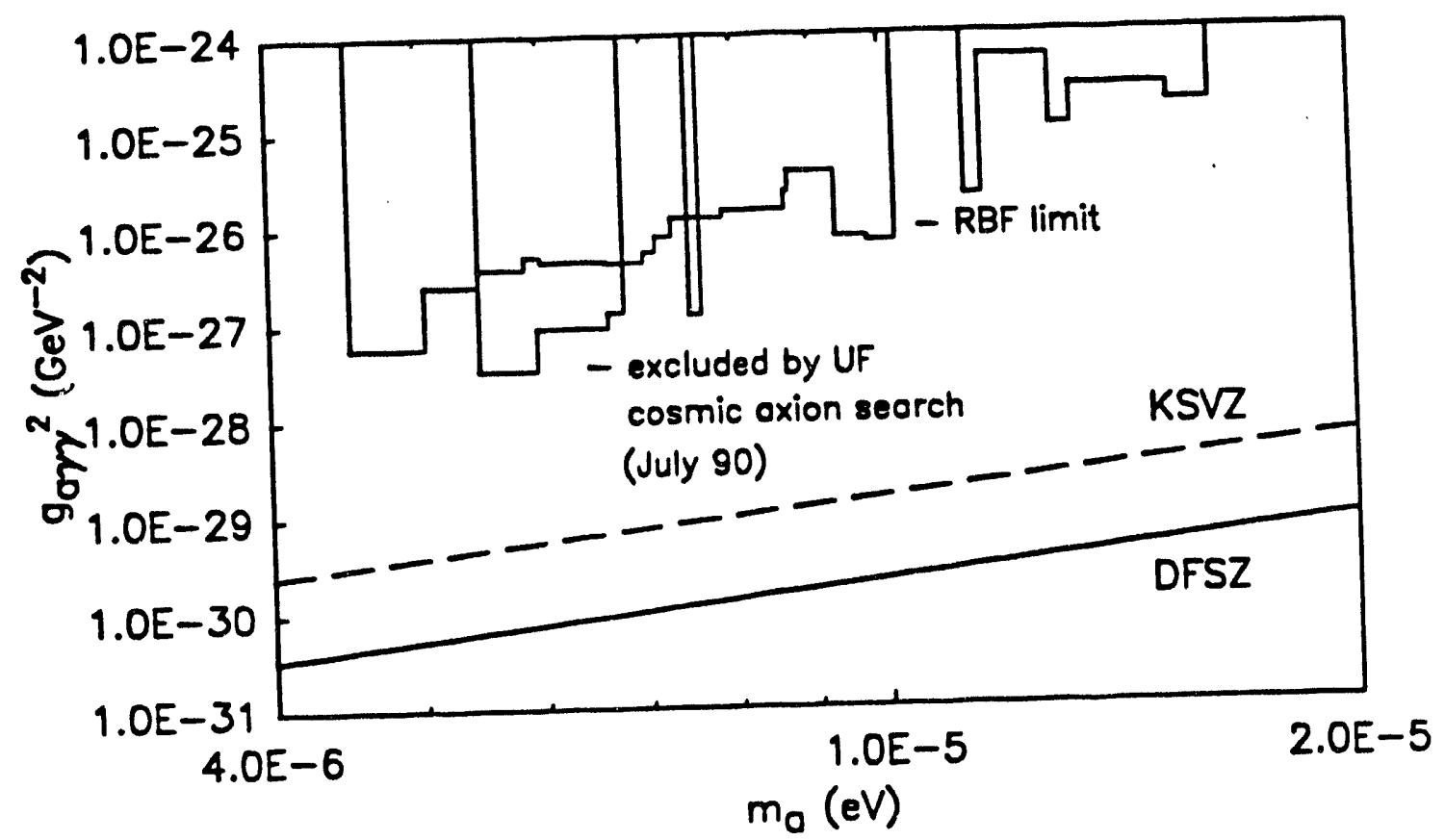

Fig. 12. Experimental limit obtained by the UF experiment on axion couplings $g_{a \gamma \gamma}^{2}$ assuming $p_{a}=p_{\text {halo }}=5 \times 10^{-25} \mathrm{~g} / \mathrm{cm}^{3}$.

$$
\Delta P_{n}=k_{B} T_{n} \mathcal{B} / \sqrt{N_{a v}}
$$

where

$$
T_{n}=T_{a m p}+T_{b a t h}
$$

is the system noise temperature, $\mathcal{B}$ is the $1 \mathrm{kHz}$ bin width and $N_{a v}$ is the number of averages.

These fluctuations set our minimum detectable signal power (at the $95 \%$ C.L.) to be

$$
P_{\text {signal }} \simeq 8 \times 10^{-22} \text { Watt }
$$

for $\mathcal{B}=1100 \mathrm{~Hz}(1 \mathrm{bin})$ and $\sqrt{2}$ times that for a signal falling into two bins. On account of the modest volume of the cavity, this noise power level is still a factor of $\sim 500$ too large for the signal expected from axion conversions, $P_{a \rightarrow \gamma} \simeq 1.3 \times 10^{-24}$ Watt in the DFSZ model assuming $\rho_{a}=\rho_{\text {halo }}$. 


\section{THE SECOND GENERATION EXPERIMENT}

The second generation experiment was approved and funded at LLNL in FY93. There are three goals for this experiment: (1) to increase the power sensitivity by a factor of 50-100 over previous experiments, (2) to take data with multiple-cavity arrays for the first time so as to extend the mass range of search to a full decade, and (3) to provide a platform where R\&D crucial to an axion experiment can be carried out.

\section{A. 8.5 Tesla Magnet}

The magnet described here is being built by Wang NMR with delivery to LLNL set for April 1994. It will be a new-build superconducting magnet and cryostat with all the latest advances in current leads, persistent-mode operation, and low-loss cryogenics. As compared to reusing existing magnets, the savings in cryogenics operating costs are expected to repay the cost of magnet fabrication in about a year.

The magnet is designed with a clear bore of $53 \mathrm{~cm}$ and an overall length of $100 \mathrm{~cm}$. The central field will be $8.5 \mathrm{Tesla}$, giving a figure of merit of $B_{0}^{2} V=11 \mathrm{~T}^{2} \mathrm{~m}^{3}$. A sketch of the magnet and cryostat is shown in Fig. 13.

The magnet will be operated in persistent mode, with the leads withdrawn to minimize the heat load on the helium bath. The time constant for decay of the current is specified as less than $10^{-7}$ per hour. The outer dewar will be heavily insulated to reduce cryogen losses as well. In $t l$ is configuration, the boiloff of liquid helium is estimated to be less than 1 liter/hour.

The inner helium reservoir is separate from the magnet helium vessel. Thermal isolation of the inner and outer reservoirs is sufficiently good that it will be possible to warm the inside space with installed heaters in order to allow the cavity and amplifiers to be removed without warming the magnet itself above $4.2 \mathrm{~K}$. Thus all cavity/amplifier exchanges are expected to be carried out with the magnet cold and energized and the inner cryostat at room temperature. (Discharging and charging the magnet take quite a bit of helium and stress the magnet significantly.)

\section{B. Cavities}

\section{Cavity design}

Cavity design for this experiment is constrained by several factors. First, the $\mathrm{TM}_{010}$ mode is the only feasible one in which to operate. In this mode, the electric field is $\vec{E}=E(x, y) \hat{z}$, along the direction of the external magnetic magnetic field, with the only nodes on the walls of the cavity. The form factor of Eqs. 14 and 15 is $C=0.69$, much larger than for any other mode. For the cylindrical cavities that we plan to use, the frequency of the $\mathrm{TM}_{010}$ mode is $f=115 \mathrm{MHz} / R[\mathrm{~m}]$, independent of cavity length. Second, for a given "central" frequency of a cavity (an empty cavity), the cavity may be tuned by 


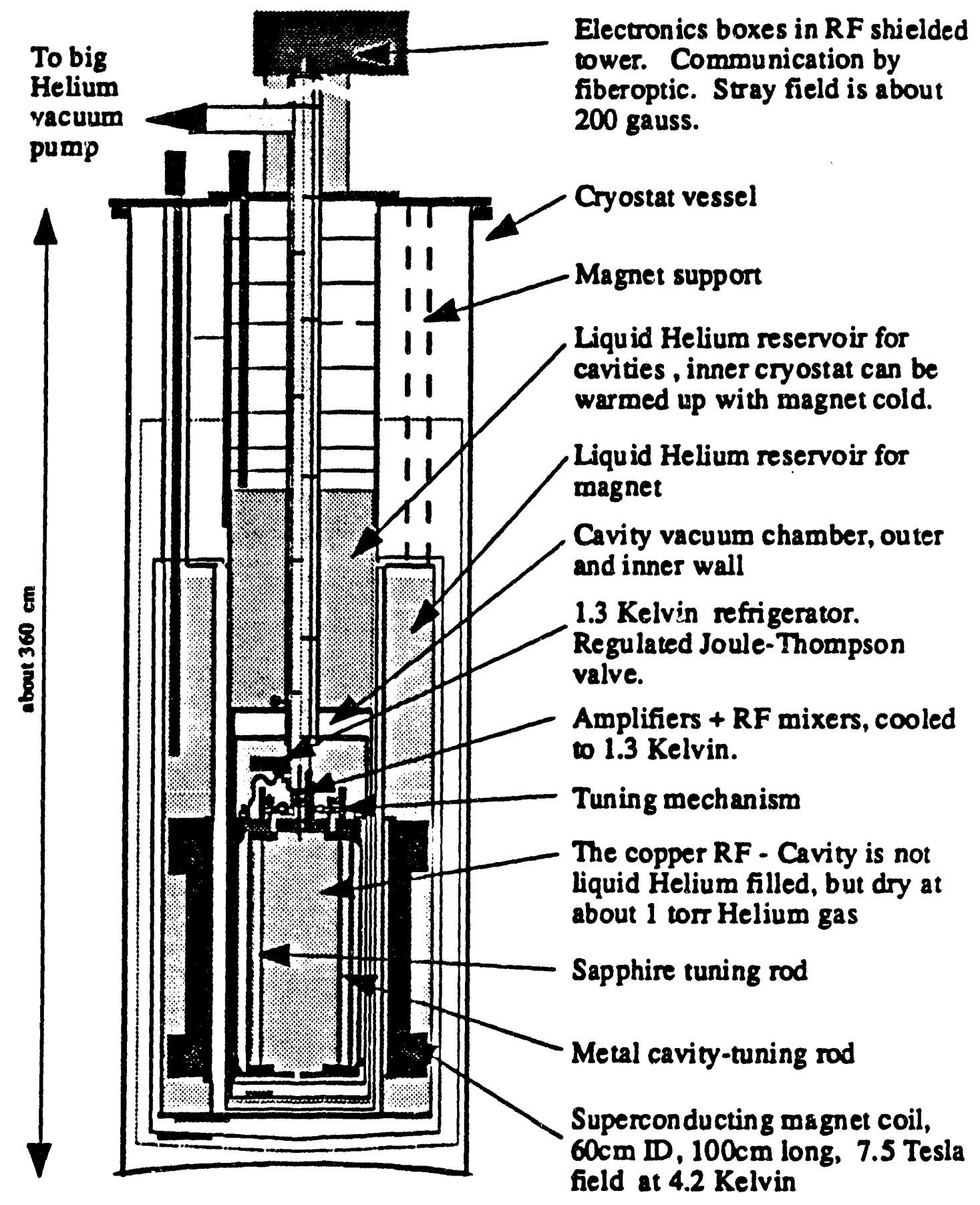

Fig. 13. Axion experiment layout.

approximately $\pm 25 \%$ without serious degradation of its $C^{2} Q$ by radial displacement of metal or dielectric tuning rods within the cavity.

Third, searching for axions in a higher mass range implies correspondingly higher frequencies, and thus cavities of smaller radii. Because the signal power for axion $\rightarrow$ photon conversion goes as $B_{z}^{2} V$, where $V$ is the volume of the cavity or cavities whereas 
the volume of a single $T M_{010}$ resonator becomes smaller as $f^{-2}$, we gain sensitivity by using as much of the magnet volume as possible by packing as many cavities as possible into the available volume. Each cavity will be tuned independently to the same frequency; cavity outputs will be combined and brought in phase to the front end of the low-noise amplifier. Because the power splitters/combiners typially combine $2^{n}$ signals into one, we have designed our cavity arrays in powers of 2 . There are two further comments we can make about this concept: (i) Power-combing cavities is not the same thing as coupling cavities; in fact, the power combiner effectively isolates the cavities from one another at the search frequency. (ii) The cryogenic microwave amplifier in the actual experiment will be in the cryostat along with the microwave cavity or cavities. (By design this will be the lowest temperature in the experiment, $T \sim 1 \mathrm{~K}$.) In this situation, the amplifier looking into a passive power combiner only sees the temperature $T$, of the isotherm, independent of the number of cavities in the array. Thus operation with multiple cavities in this experiment will incur no different physical noise temperature than for a single cavity.

The experiment will start with a single cavity, filling the magnet bore. Next, a partition will be used to divide the cavity into two cavities, each of semicircular cross section. Each half has a $\mathrm{TM}_{010}$ resonant frequency $1.56 \times$ that of the single cylindrical cavity. The field pattern for such a divided cavity is shown in Fig. 14.

Four cavities can be obtained by further subdividing the cylinder into quarter-circular sections. The field pattern for this configuration, which resonates at $2.1 \times$ the frequency of the cylindrical cavity, is shown in Fig. 15. Note that both the two- and four-cavity arrangement use almost all of the magnet volume.

Next would come eight cavities in a nearly close-packed array, with seven cavities around the outer edge of the magnet bore and one in the center. This configuration uses about $73 \%$ of the magnet volume, and gives a resonant frequency $3.3 \times$ that of a single cavity.

The array of 16 cavities in a transverse section will consist of 16 circular cylinders, hexagonally-close-packed. However, on account of the problems with mode localization that occur with very long cavities, ${ }^{30}$ we wish to longitudinally segment this array into two clusters of 16 , end to end.

This sequence of cavities covers $380 \mathrm{MHz}-2.7 \mathrm{GHz}$, corresponding to axion masses of 1.5-11 $\mu \mathrm{eV}$. Details on the design of an experiment with a large number of cavities may be found in Ref. 44. Table 1 shows cavities which would be employed. The first column gives the total number of cavities, the second the arrangement, the third the radius of the cylinders with respect to that of the magnet bore, the next the fraction of the magnet bore which would be cavity space. The last two columns give the center frequency and expected tuning range of each cavity set. 


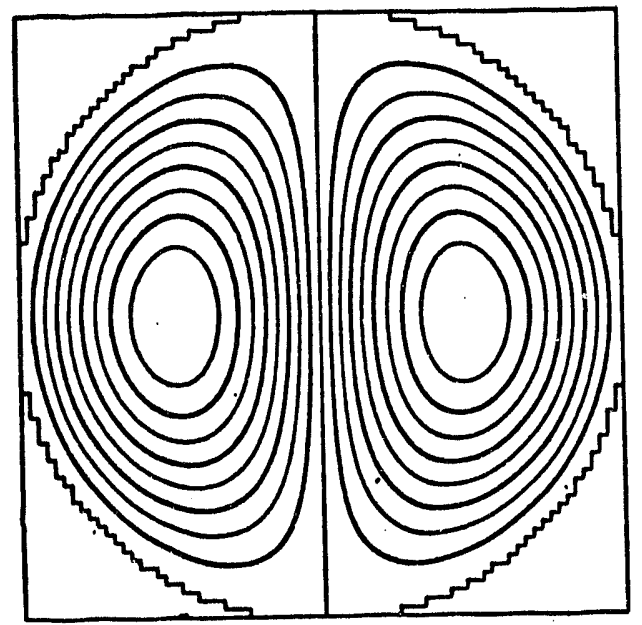

Fig. 14. Field pattern for a pair of cavities with semi-circular cross section.

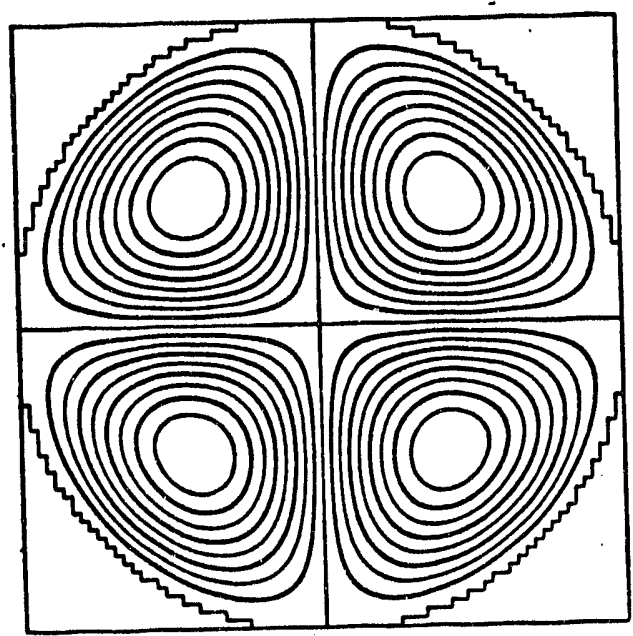

Fig. 15. Field pattern for four cavities with quarter-circular cross section.

Table 1. Parameters of multicell cavities.

\begin{tabular}{|c|c|c|c|c|c|}
\hline \multirow{2}{*}{$\begin{array}{l}\text { Number } \\
\text { of cavities }\end{array}$} & \multirow[t]{2}{*}{ Arrangement } & \multirow[t]{2}{*}{$r / R$} & \multirow{2}{*}{$\begin{array}{l}\text { Packing } \\
\text { fraction }\end{array}$} & \multicolumn{2}{|c|}{ Tuning Range } \\
\hline & & & & $\mu \mathrm{eV}$ & $\mathrm{MHz}$ \\
\hline 1 & 0 & 1 & 1 & $1.6-2.5$ & $377-595$ \\
\hline 2 & $\theta$ & 1 & 1 & $2.5-3.4$ & $595-830$ \\
\hline 4 & $\oplus$ & 1 & 1 & $3.4-5.1$ & $830-1246$ \\
\hline 8 & $0 \times 8$ & 0.30 & 0.73 & $5.1-7.2$ & $1246-1752$ \\
\hline 32 & $0 \times 16 \times 2$ & 0.22 & 0.75 & $7.2-11$ & $770-274$ \\
\hline
\end{tabular}




\section{Cavity fabrication}

The two pilot experiments utilized small single cavities, which were made of oxygenfree high-conductivity (OFHC) copper. The second generation experiment will require several cavity arrays, consisting of multiple cavities, each larger than those used in the pilot experiments. Fabricating the cavities for the large-scale experiment from OFHC tube stock thus may not be optimal in view of cost, weight and rigidity.

Almost two years ago, a group from the Institute for Nuclear Research of the Russian Academy of Sciences, headed by Dr. Nilolai A. Golubev, joined our collaboration, proposing to take complete responsibility for fabricating all the microwave cavities for the experiment. Furthermore, they suggested a novel technology for fabricating the cavities, namely metallized precision formed ceramic. Ceramic cavities are light, strong, and require a minimum of machining after being fired to hold mechanical tolerances. INR has long experience in metallized ceramics, principally for beam pipes in rapid cycling synchrotrons.

The use of multiple cavities is crucial to the experiment. To search for axions higher in mass, the radius of a single microwave cavities goes down as the inverse of the axion mass. Therefore, to take maximum advantage of the magnet $B_{z}^{2} V$, it is essential to use arrays of cavities, each cavity being independently tunable, with the outputs combined in power. Neither of the pilot experiments ever ran with more than one cavity. As a final chapter to his thesis, however, C. Hagmann ${ }^{33}$ successfully demonstrated the principle of combining the output from two cavities in a test at $300 \mathrm{~K}$. The magnitude and shape of the $Q$ curve of the combined cavities closely matched theory.

Two INR physicists visited Livermore for the month of September 1992, where the 4-cavity array was prepared for testing at $4.2 \mathrm{~K}$ in a $50 \mathrm{~cm}$ i.d. LHe dewar in the magnetic fusion cryogenics lab. This array is already of the full size that will be used in the actual experiment. One (N. Golubev) spent October at the University of Florida, and measured the $Q$ at low temperature of a copper-plated ceramic cavity.

\section{Cavity tuning}

The cavities will be tuned by moving either a dielectric rod or metal rod radially inwards from the outer wall to the center. With either rod located near the wall, the frequency is close to that of an empty cavity. The dielectric rod gives a decrease in resonarit frequency as it moves to the center; the metal rod an increase. Our simulations ${ }^{30}$ and measurements ${ }^{31}$ indicate that roughly a $20 \%$ decrease and $30 \%$ increase from the empty cavity resonance can be achieved without too serious a loss in $C^{2} Q$. Figs. 16 and 17 show the changes of frequency that occur with sideways motion of a dielectric and a metal rod respectively.

Note that dielectric rods big enough $(6-8 \mathrm{~cm}$ diameter) to tune the larger cavities are not readily available. Therefore we plan to use bundles of smaller $(0.6-1 \mathrm{~cm}$ diameter $)$ rods 


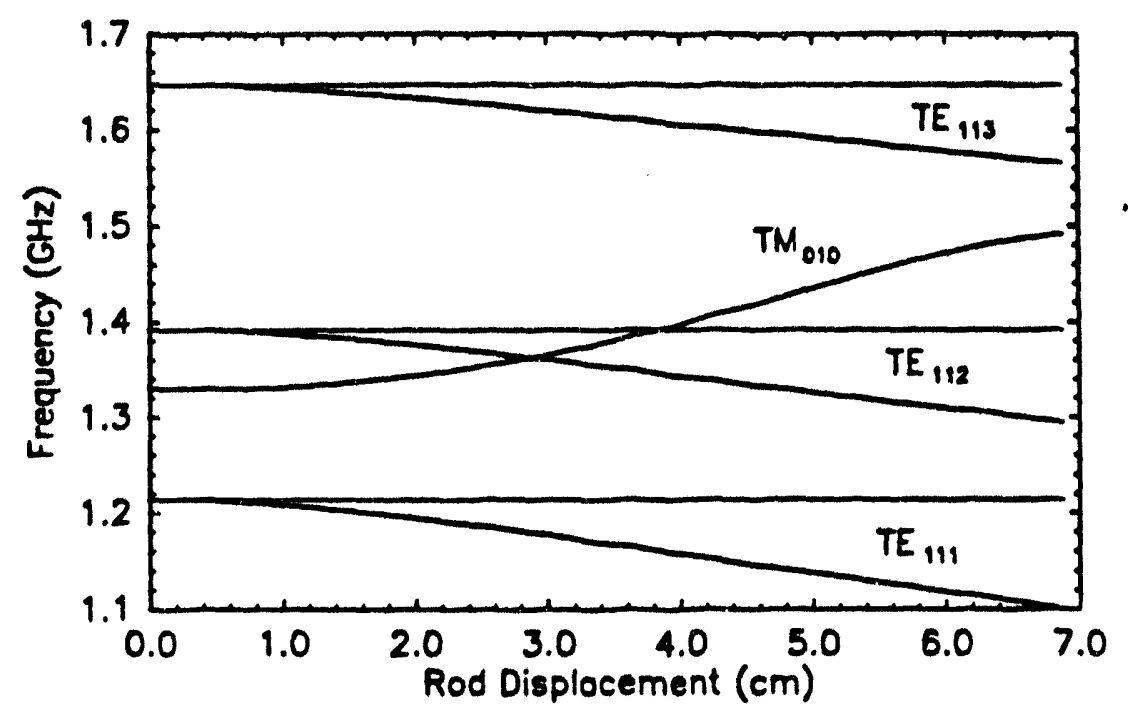

Fig. 16. Cavity frequency vessus sadial displacement of a dielectric rod. $(R=7.62 \mathrm{~cm}, r=0.635$ $\mathrm{cm}$, and " = 9.5.) The $T M_{010}$ mode used in the search is shown, along with nearby $T E_{21 \ell}$ modes. Note the lifting of the degeneracy of the $\mathrm{TE}_{13 \ell}$ modes as the rod is moved sideways.

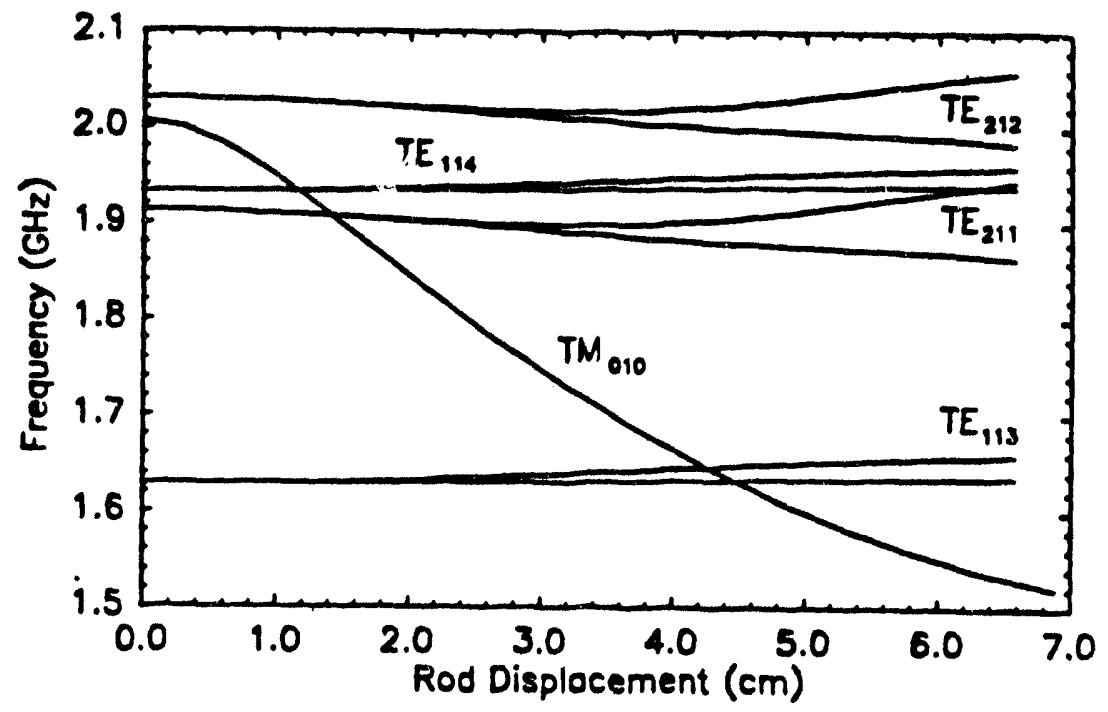

Fig. 17. Cavity frequency versus radial displacement of a metal rod. $(R=7.62 \mathrm{~cm}$ and $r=0.622$ cm.) The TMO10 mode used in the search is shown, along with nearby TE modes.

to make up the tuning assembly. Alumina ceramic rods in these diameters are available from at least two suppliers. The $2.2 \mathrm{~m}$ length needed appears to pose no problems. An advantage of using the bundles is that the rods can be rebundled and used in several of the cavities.

The multi-cavity arrays will work only if all are at the same frequency. We plan to use one of the cavities as the "master" and the remainder as "slaves." The resonance of the master will be set and its $Q$ determined just as is now done with a single cavity. Then 
each of the slave cavities will be adjusted to maximize its transmission at the resonant frequency of the master cavity. Peaking up the signal in this way should be a relatively rapid procedure. Once the cavities are synchronized they should stay together for a number of spectra, so long as the tuning rod mechanisms are sufficiently precise.

\section{Cavity Tolerances}

The search rate at a particular sensitivity $(s / n)$ goes as $C^{2}$, where $C$ is the cavity formfactor ${ }^{30}$ It is therefore crucial to keep $C$ as close as possible to its theoretical maximum ( 0.69 for the TM010 mode in an empty cavity). Perturbations or non-uniformities which break translational symmetry of the cavities, however, can lead to mode-localization of the TM010 mode in the cavity, and degrade $C$, perhaps seriously.

The maximum allowable reduction of the form factor $C$ by mode-localization for any reason can be translated into a general condition on the "local frequency" along the 2 -axis (axis of translational symmetry)

$$
\frac{\delta \omega}{\omega}<\frac{2}{\omega^{2} L^{2}}
$$

where $\omega$ is the angular frequency $=2 \pi f$, and $L$ the length of the cavity. This inequality must be satisfied if $C^{2}$ is not to be diminished by more than $10 \%$.

Because $\omega \sim 1 / R$, the mode-localization condition becomes one on the accuracy of the cavity radius. $\delta R / R<0.7(R / L)^{2}$. Small deformations of the cavity body which preserve the cavity perimeter (squeezing the cavity out-of-round slightly) also preserve the crosssectional area and the frequency to first order, and thus are not a problem. Meeting the tolerances through the array of 16 cavities in a transverse cut will be straightforward: the cavity radius must be held to $\sim 0.7 \mathrm{~mm}$ tolerance.

A simple diagnostic test will be used to determine whether a particular cavity suffers from mode localization: measure the end-to-end transmission of the cavity at the resonant frequency. If the coupling ports at each end are critically coupled, then the cavity should transmit nearly $100 \%$ at resonance if it is performing properly. If the cavity is badly localized, then the transmission is substantially decreased.

\section{Tuning and combining cavity outputs}

In the multi-cavity detector, the outputs of all the cavities need to be brought in phase to the input of the microwave amplifier. This means that

1. all the cavities must have the same resonant frequency,

2. the coupling $Q$ (i.e., $Q_{h}$ ) must be the same for all the cavities, and

3. the length of RF cable between each cavity and the power combiner must be the same. Power splitters/combiners are used to combine the signals into a single coaxial line. These passive devices transmit all of the power incident on them when the individual input signals have equal amplitude and phase. Otherwise, some of the incident power is lost in a termination. 
Although this appears a difficult task, we have demonstrated that cavity outputs can be combined easily and completely, by making a a test of this method for the simplest case of two cavities. The test setup is shown in Fig. 18. The empty cavities had $\mathrm{TM}_{010}$ resonance frequencies of $1.6 \% \mathrm{GHz}$ with $Q_{\text {wall }} \sim 3 \times 10^{4}$. The frequencies differed initially from each other by $0.7 \%$, but could be made to match by inserting a small Tefion tuning rod $(0.4 \mathrm{~cm}$ in diameter) into one of the carities.

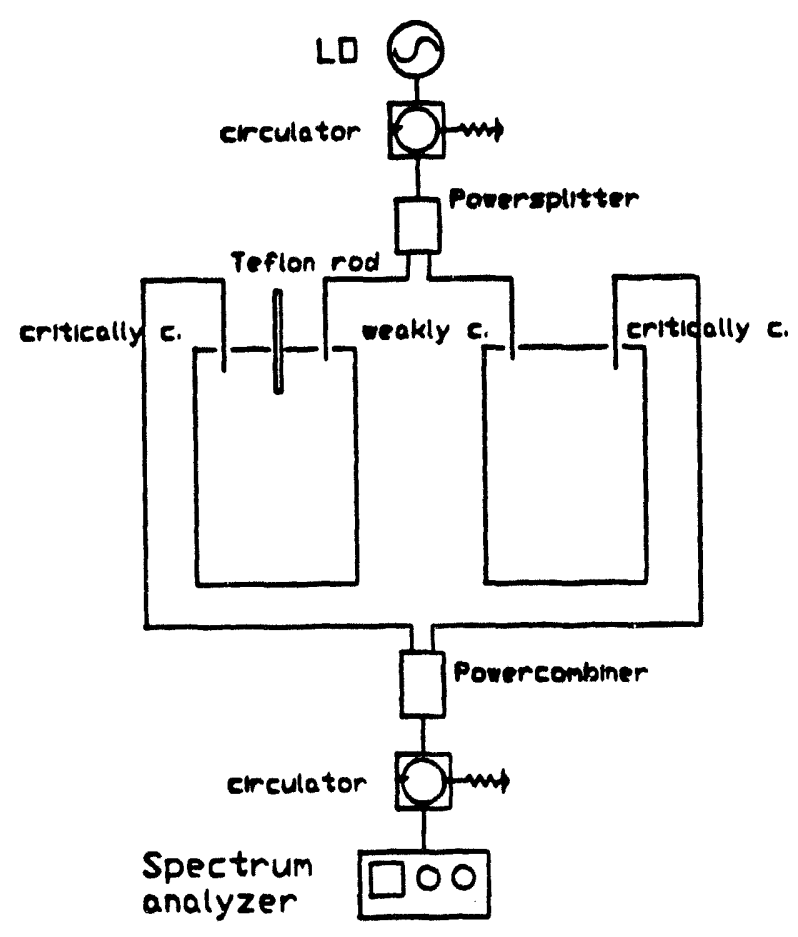

Fig. 18. Test setup of two frequency-matched cavities.

Two identical Wilkinson power dividers/combiners ${ }^{45}$ were used to split and combine the signals in phase. A signal of voltage $V \sim e^{-i \omega t}$ entering the divider is split into equal amplitude signals $V \sim e^{-i \omega t} / \sqrt{2}$ at the output. When used as a combiner with amplitudes $a e^{-i \omega t}$ and $b e^{-i \omega t-i \phi}$, the output voltage is $\left(a+b e^{-i \phi}\right) e^{-i \omega t} / \sqrt{2}$ and power is dissipated in internal resistors unless the two inputs are exactly balanced.

In the experiment, one of the ports of each cavity was critically coupled, while the other was weakly coupled. The cavity can be modeled by a series $R L C$-circuit with resonance frequency $\omega_{0}^{2}=1 / L C$ and $Q_{w}=\omega_{0} L / R$. The input impedance of a single port cavity near resonance is given by

$$
Z_{c}=\frac{Z_{0}}{\beta R}\left(R+i \omega L+\frac{1}{i \omega C}\right) \simeq \frac{Z_{0}}{\beta}\left(2 i Q_{w} \frac{\omega-\omega_{0}}{\omega_{0}}+1\right)
$$

where $\beta=Q_{w} / Q_{h}$ is the coupling parameter and $Z_{0}$ is the characteristic line impedance, usually $50 \Omega$. The input impedance is equal to the line impedance for a critically coupled 
cavity $(\beta=1)$ on resonance, making the cavity reflectionless. For a two-port cavity, Eq. 25 still applies but $R$ is modified to include the additional losses from the second port. A signal transmitted through a critically coupled cavity (while the second port is weakly coupled) will suffer a phase shift $V \sim e^{i \alpha}$ with

$$
\tan \alpha=\left(\frac{1}{\omega C}-\omega L\right) / 2 R \simeq 2 Q_{L} \frac{\omega-\omega_{0}}{\omega_{0}} \equiv x
$$

and the amplitude is decreased by a factor $1 / \sqrt{1+x^{2}}$ relative to the value on resonance.

The output voltage of the power combiner for the Local Oscillator (LO) frequency at the resonance frequency of the first cavity and variable resonance frequency of the second cavity is then

$$
V=\frac{V_{0}}{\sqrt{2}}\left(\frac{1}{\sqrt{2}}+\frac{1}{\sqrt{2}} \frac{1}{\sqrt{1+x^{2}}} e^{i \alpha}\right)
$$

and the power

$$
\left|\frac{V}{V_{0}}\right|^{2}=\frac{1}{4} \cdot\left(1+\frac{3}{1+x^{2}}\right)
$$

This relationship is plotted in Fig. 19. Also shown are the measured values, which are in good agreement with the theoretical curve. Operation of a multi-cavity detector would require keeping the cavity resonance frequencies within a few percent of the cavity width in order to avoid mismatch losses. This test demonstrates the feasibility of using phased multiple cavities as axion cavity detectors.

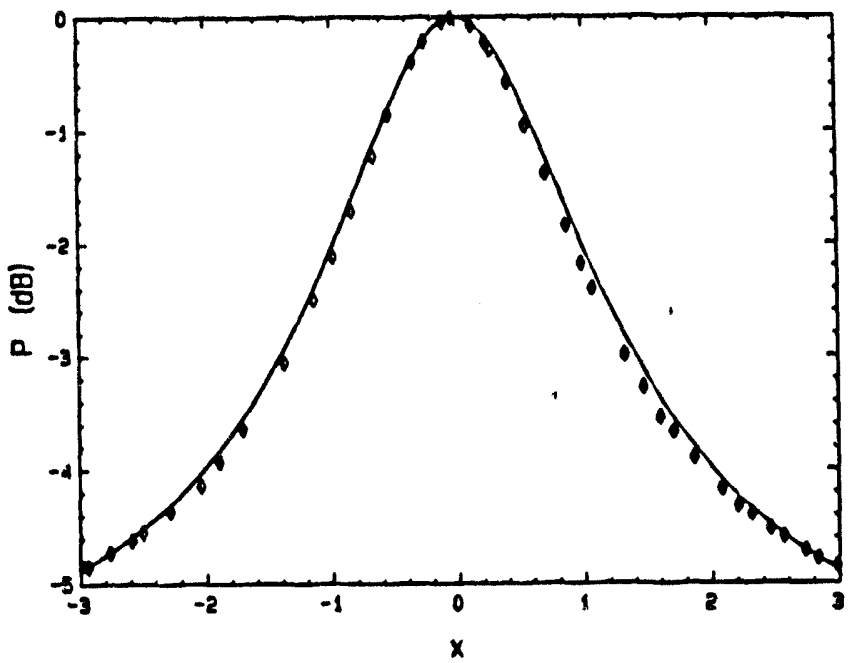

Fig. 19. Transmitted power for the configuration shown in Fig. 18.

It should be emphasized that we are not coupling cavities, but power-combining them. A direct coupling of the cavities with mode mixing and splitting into symmetric 
and antisymmetric modes was not observed in the original arrangement. This was due to the large isolation ( $>30 \mathrm{~dB}$ ) between the power combiner arms. It was observed however when the strongly coupled ports were directly connected with a coax cable.

The calculation agrees with the measurement within $\pm 0.2 \mathrm{~dB}$, with the difference largely due to the uncertainty in determining the variable resonance frequency of the second cavity. Note that when the two cavities were synchronized, the calculation and measurement agree extremely well. (A correction has been made for the $0.1-0.2 \mathrm{~dB}$ loss in the power combiner.)

\section{Electronics}

\section{Cryogenic amplifier}

We plan to use cryogenic microwave amplifiers manufactured by Berkshire Technologies, ${ }^{38}$ or other sources. According to Berkshire, HEMT amplifiers with excess noise temperatures $\sim 5 \mathrm{~K}$ can be made over the $200 \mathrm{MHz}-1 \mathrm{GHz}$ range. Between 1 and 2 $\mathrm{GHz}$, noise temperatures around $3-4 \mathrm{~K}$ are available. ${ }^{31}$ (See Fig. 8 and Fig. 9.) At higher frequencies the noise temperature will increase, reaching around $8 \mathrm{~K}$ at $4 \mathrm{GHz}$.

Equation 17 shows that considerable improvement in search rate or sensitivity can be achieved by reducing the amplifier noise temperature. Technology is improving rapidly in this area, and we anticipate being able to take considerable advantage of this as the experiment continues. Figure 20 shows the noise temperature that is currently achievable in low-noise GaAs HEMT amplifiers. ${ }^{38}$ Currently, amplifiers have noise temperatures that are about half what was available when the pilot experiment ai UF was constructed.

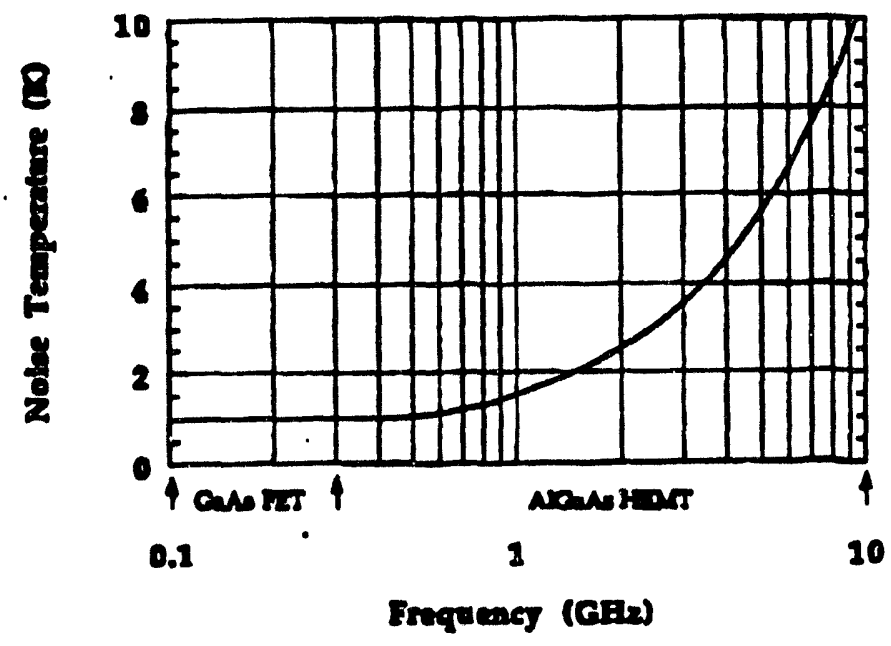

Fig. 20. Current state-of-the-art in amplifier noise temperature. 


\section{Receiver}

The remainder of the experimental setup will be as shown in Fig. 3: a double superheterodyne receiver, digital spectrum analyzer, and computer control. The output of the cryogenic amplifier will be further amplified by a room temperature amplifier and converted to the $10.7 \mathrm{MHz}$ first IF frequency by an image reject mixer. The second converter will use a double-balanced mixer, providing an audio signal spanning $0-30 \mathrm{kHz}$ which will undergo a final amplification before $A / D$ conversion. We will use a digital fast Fourier transform (FFT) scheme to spectrum analyze the signal from the cavity. Each spectrum wil. be searched for $2 \sigma$ peaks in single bins and combination of neighboring bins. If a peak is found, another set of spectra will be taken and averaged with the first. If the peak remains statistically significant for several retries, the peak will be flagged for later investigation.

\section{Search rate}

The search rate for the experiment is given in Eq. 17. If we evaluate Eq. 17 with the parameters of the second generation experiment, $V=0.2 \mathrm{~m}^{3},\langle B\rangle=7.5 \mathrm{~T}, T_{n}=1 \mathrm{~K}$, and $Q_{w}=Q_{a} / 5$, we find that the search rate is approximately $100 \mathrm{MHz} / \mathrm{yr}$ at the DFSZ value of $g_{\gamma}$. The actual searcin rate will be less than this because there are several factors in the experiment which are not included in Eq. 17. First, because many (approximately $80 \%$ ) of the spectra have positive peaks exceeding $2 \sigma$ and are repeated, rechecking nearly doubles the scan time. Second, adjacent spectra are overlapped by about $20 \%$, in order not to miss a signal which was right at the end of the spectrum. Third, the cavity resonant frequency and $Q$ need to be measured; multiple cavities will need to be synchronized, etc. When the number of cavities is small, this is not more than a $1-5 \%$ overhead; however, for 32 cavities we estimate this overhead to be $\approx 25 \%$. Fourth, a period of -overal days to a week will go by when cavity assemblies are being changed. From these considerations, we have concluded that the second-generation experiment will not be able to reach the DFSZ limit; the magnetic volume is simply not large enough.

However, a more optimistic estimate is obtained if one takes the KFSZ value for $g_{\gamma}$, where the coupling of axions to two photons is larger. Use of this value, $g_{\gamma}=0.97$, leads to a scan rate of more than $1 \mathrm{GHz} /$ year (or $1 / f d f / d t=3 \% /$ week) at the low frequency end of the tuning range. Our simulations indicate that the entire range in Table 1 could be scanned in about three years. Thus, this second generation experiment is the first axion detector that has the sensitivity to detect or rule out axions at cosmologically significant values of model parameters. Figure 21 summ sizes the sensitivity of this experiment. This figure shows the current limits on $g_{a \gamma \gamma}$ set by the RBF and UF axion searches along with the region that would be covered by this experiment. Also shown are lines corresponding to two choices of Peccei-Quinn charges within the class of hadronic axion models, both well within the sensitivity of this experiment. The increase in sensitivity and in range of the proposed experiment is substantial. 


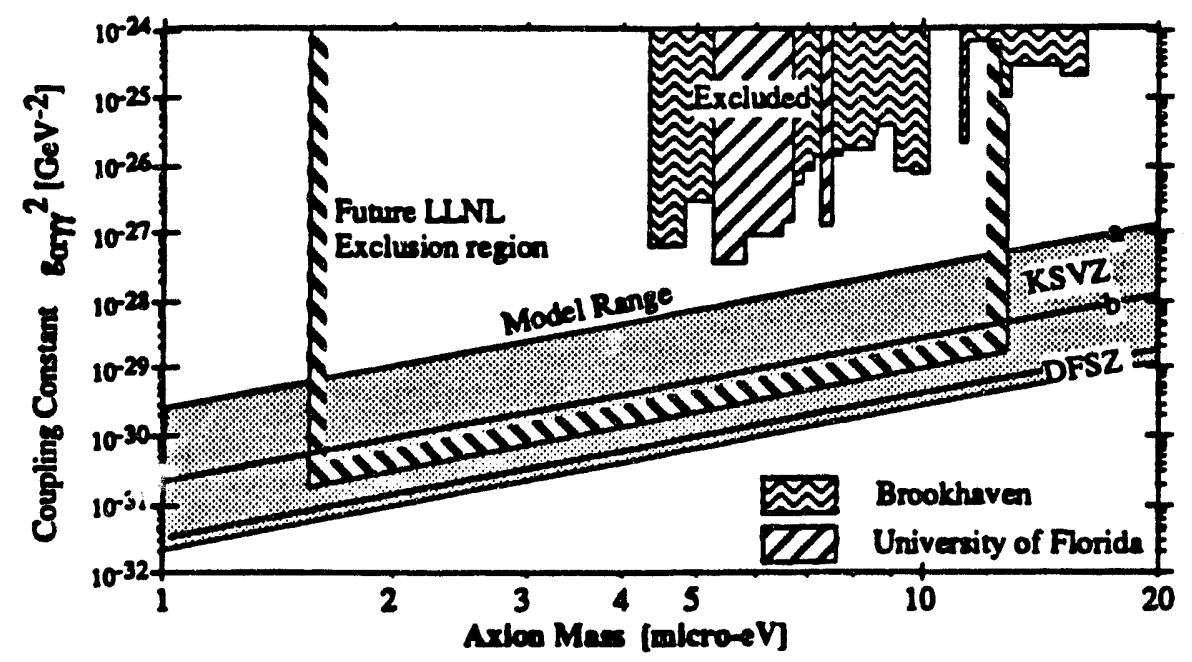

Fig. 21. Experimental limits on the electromagnetic coupling $g_{a \gamma \gamma}$ of the axion. Values in the shaded areas at upper right have been excluded by the RBF and UF sec iches. The large crosshatched region gives the region were the axion would either be discovered with a signal/noise ration of 4 or ruled out at the $97 \%$ confidence level by the second-generation experiment.

\section{References}

1. The Decade of Discovery in Astronomy and Astrophysics, National Research Council (National Academy Press, Washington, 1991).

2. R.D. Peccei and H. Quinn, Phys. Rev. Lett. 38, 1440 (1977); Phys. Rev. D 16, 1791 (1977).

3. S. Weinberg, Phys. Rev. Lett. 40, 223

F. Wilczek, Phys. Rev. Lett. 40, 279 (1977).

4. Recent reviews include: (a) J.E. Kim, Phys. Rep. 150, 1 (1987); (b) H.Y. Cheng, Phys. Rep. 158, 1 (1988); (c) R.D. Peccei, The Strong CP problem, in CP Violation, edited by C. Jarlskog (World Scientific, 1989); (d) M. Turner, Phys. Rep. 197, 67 (1990).

5. G. 't Hooft, Phys. Rev. Lett. 37, 8 (1976) and Phys. Rev. D 14, 3432 (1976); R. Jackiw and C. Rebbi, Phys. Rev. Lett. 37, 172 (1976); C. G. Callan, R. F. Dashen and D. J. Gross, Phys. Lett. 63B 334 (1976).

6. A. A. Belavin, A. M. Polyakov, A. S. Schwartz and Y. S. Tyuplin, Phys. Lett. 59B, 85 (1975).

7. S. Weinberg, Phys. Rev. D 11, 3583 (1975).

8. I. S. Altev et al., Phys. Lett. B136, 327 (1984).

9. V. Baluni, Phys. Rev. D 19, 2227 (1979); R. J. Crewther, P. Di Vecchia, G. Veneziano and F. Witten, Phys. Lett. 88B, 123 (1979).

10. M. Kobayashi and K. Maskawa, Progr. Theor. Phys. 49, 652 (1973). 
11. W. A. Bardeen and S.-H. H. Tye, Phys. Lett. 74B, 229 (1978); J. Ellis and M. K. Gaillard, Phys. Lett. 74B, 374 (1978); T. W. Donnelly et al., Phys. Rev. D 18, 1607 (1978). More recent treatments include: M. Srednicki, Nucl. Phys. B260, 689 (1985); P. Sikivie, in Cosmology and Particle Physics, ed. by E. Alvarez et.al., (World Scientific, 1987) p. 143.

12. H. Georgi, H. R. Quinn and S. Weinberg, Phys. Rev. Lett. 33, 451 (1974).

13. M. Dine, W. Fischler and M. Srednicki, Phys. Lett. 104B, 199 (1981); A. P. Zhitnitskii, Sov. J. Nucl. Phys. 31, 260 (1980).

14. J. Kim, Phys. Rev. Lett. 40, 223 (1977); M.A. Shifman, A.I. Vainshtein and V.I. Zakharov, Nucl. Phys. B166, 493 (1980).

15. Recent reviews include: M. S. Turner, Phys. Rep. 19767 (1990); G. G. Raffelt, Phys. Rep. 1981 (1990).

16. G. Raffelt and D. Seckel, Phys. Rev. Lett. 60, 1793 (1988); M. S. Turner, Phys. Rev. Lett. 60, 1797 (1988); R. Mayle et al., Phys. Lett. 203B, 199 (1988); T. Hatsuda and M. Yoshimura, Phys. Lett. 203B, 469 (1988); A. Burrows, M. S. Turner and R. P. Brinkman, Phys. Rev. D 39, 1020 (1989); N. Iwamoto, Phys. Rev. D 39, 2120 (1989); A. Burrows, M.T. Ressell, and M.S. Turner, Plıys. Rev. D42 3297 (1990).

17. J. Preskill, M. Wise and F. Wilczek, Phys. Lett. 120B, 127 (1983); L. Abbott and P. Sikivie, Phys. Lett. 120B, 133 (1983); M. Dine and W. Fischler, Phys. Lett. 120B, 137 (1983).

18. R. Davis, Phys. Lett. 180B, 225 (1986); R. Davis and E. P. S. Shellard, Nucl. Phys. B324, 167 (1989); A. Dabholkar and J. M. Quashnock, Nucl. Phys. B333, 815 (1990).

19. D. Harari and P. Sikivie, Phys. Lett. 195B, 361 (1987); C. Hagmann and P. Sikivie, Nucl. Phys. B363, 247 (1991).

20. P. J. Steinhardt and M. S. Turner, Phys. Lett. B129, 51 (1983).

21. W. G. Unruh and R. M. Wald, Phys. Rev. D 32, 831 (1985); T. De Grand, T. W. Kephart and T. J. Weiler, Phys. Rev. D 33, 910 (1986).

22. M. Turner and F. Wilczek, Phys. Rev. Lett. 66 (1991) 5; A. Linde, Phys. Lett. B259 (1991) 38.

23. J. Ipser and P. Sikivie, Phys. Rev. Lett. 50, 925 (1983); M.S. Turner, F. Wilczek, and A. Zee, Phys. Lett. B125, 35 (1984).

24. M. S. Turner, Phys. Rev. D 33, 889 (1986).

25. M. Turner, Phys. Rev. D 42, 3572 (1990).

26. P. Sikivie and J. R. Ipser, Phys. Lett. B291, 288 (1992).

27. P. Sikivie, Phys. Rev. Lett. 51, 1415 (1983). 
28. P. Sikivie, Phys. Rev. D 32, 2988 (1985); L. Krauss, J. Moody, F. Wilczek and D. Morris, Phys. Rev. Lett. 55, 1797 (1985).

29. S. DePanfilis, A.C. Melissinos, B.E. Moskowitz, J.T. Rogers Y.K. Semertzidis, W.U. Wuensch, H.J. Halama, A.G. Prodell, W.B. Fowler and F.A. Nesrick, Phys. Rev. Lett. 59, 839 (1987).

30. C. Hagmann, P. Sikivie, N.S. Sullivan and D.B. Tanner, Rev. Sci. Instrum. 61, 1076 (1990).

31. C. Hagmann, P. Sikivie, N.S. Sullivan and D.B. Tanner, Phys. Rev. D 42, 1297 (1990).

32. D. Morris, private communication.

33. C. Hagmann, Ph.D thesis, University of Florida, 1990, unpublished.

34. W.U. Wuensch, S. DePanfilis-Wuensch, Y.K. Semertzidis, J.T. Rogers, A.C. Melissinos, H.J. Halama, B.E. Moskowitz, A.G. Prodell, W.B. Fowler, and F.A. Nezrick, Phys. Rev. D 40, 3153 (1989).

35. A.C. Melissinos, B.E. Moskowitz, J.T. Rogers, Y.K. Semertzidis, S. DePanfilisWuensch, W.U. Wuensch, H.J. Halama, A.G. Prodell, W.B. Fowler, and F.A. Nezrick, in Cosmic Axions edited by C. Jones and A. Melissinos (World Scientific, Singapore, 1990), p. 39.

36. C. Hagmann, P. Sikivie, N.S. Sullivan and D.B. Tanner, in Cosmic Axions edited by C. Jones and A. Melissinos (World Scientific, Singapore, 1990), p. 42.

37. Trans-Tech Inc., Adamstown, MD 21710.

38. Berkshire Technologies Inc., Oakland, CA 94609.

39. W.R. McGrath, A.V. Räisänen and P.L. Richards, Int. J. Infrared and Millimeter Waves 7, 543 (1986).

40. Passive Microwave Technology, Camarillo, CA. The models used were LTA1219KS (1.3-1.7 GHz) and LTA1267KS (1.7-2.2 GHz).

41. Chimera system with AD16 option, Atlanta Signal Processors Inc., Atlanta, GA 30308.

42. Texas Instruments TMS320C25 digital signal processor.

43. Our limit is not valid in the narrow window $1.346096-1.346382 \mathrm{GHz}$, where a mode crossing occurred and where no data could be obtained.

44. K. van Bibber et al., Proc. of the Workshop on Astroparticle Physics, edited by D. Cline and R. Peccei (World Scientific, Singapore, 1992) p. 154; K. van Bibber et al., Particles and Fields '91, edited by D. Axen, D. Bryman, and M. Comyn (World Scientific, Singapore, 1992) p. 992.

45. Triangle Microwave, E. Hanover, NJ. 
Computer Acquisition for Research in Theoretical and Experimental High Energy Physics

\section{Task S}

Principal Investigators: Paul Avery and John Yelton

Physics Department

University of Florida

Gainesville, Florida 32611 


\begin{abstract}
We present a proposal to DOE to upgrade and maintain the HEP computing facilities at the University of Florida. Our request for funds has two parts: (1) a special, one shot upgrade that would replace our existing DECstation based system with a configuration based on DEC Alpha processors, fast disk and a very fast network switch and (2) continuation of support for students, a system manager/programmer and maintenance. The funding for the upgrade comes from the University, DEC, DOE and the HPCC program at NSF and is part of an ambitious project involving UF, Cornell, UCSD, Carleton University, Xerox and DEC.
\end{abstract}

\title{
I Introduction
}

This computing proposal (Task S) is submitted separately from the CLEO portion of our DOE proposal (Task B) and dates from the time we submitted a proposal to DOE in 1987-88 to acquire a new computer system. Since that time we have become involved with many computing initiatives which justify the submission of separate proposals.

\section{History}

Our computing system has undergone many changes. We had for several years (1986-1988) a single Microvax II (2 MB) with 1.4 Gbytes of disk and a tape for our CLEO use. Money for this machine was provided by a $\$ 25 \mathrm{~K}$ competitive award at UF based on our proposed work on distributed computing. The Microvax II allowed us to do software development for CLEO and DO and permitted local data analysis using the large (for its time!) disk resources.

In 1988 or so we put together a package of $\$ 400 \mathrm{~K}$ (50\% UF and $50 \%$ DOE) to acquire a VAX 6220 with 10 Gbytes of disk, 6250 tape, 5 VAX 3100 desktop workstations and an 8 node VAX 3200 computing farm. We signed an External Reseach Proposal (ERP) with DEC which brought us contacts with DEC engineers and a $50 \%$ price reduction on all hardware components. The deal with DEC, UF and DOE was based on our distributed computing system UFMulti which we had just developed for Vax systems. This software allowed us to run single jobs on the whole computing farm, giving us access to more computing than what was available at Cornell. Andy White, who was at UF at the time, also ran a great deal of DO and SSC simulations on the farm.

In 1990, we traded in the VAX 6220, 10 Gbytes disk, 6250 bpi tape and the 8 node Vax farm for $28 \mathrm{DECstation} 5000$ s (16 MB), 17 of them networked with FDDI. This deal involved a - cond ERP with DEC and cost us nothing for the DECstations since we negotiated a large price allowance for the VAX trade in. We then purchased two VAX 3100 servers to replace the expensive 6220 and a lot of disk, along with several $8 \mathrm{~mm}$ tape drives. We also hired students to put UFMulti into Unix, a necessary move. UFMulti has been used now for almost three years to distribute jobs across the DECstations. In the meantime we have purchased three more 
DECstations, additional disk, PCs and two Next machines, the latter as part of an effort to collaborate on GISMO, the new Monte Carlo package being developed at SLAC. We are not directly involved in that effort now.

\section{Upgrade plans}

Our present system, described in detail later, is still fairly advanced and we have performed a large amount of computing with it on behalf of CLEO. However, we have an opportunity to expand the system even further using the new DEC Alpha processors which we can acquire for low prices. We propose to use $\$ 150 \mathrm{~K}$ in UF funds and $\$ 50 \mathrm{~K}$ in additional DOE funds (this year only) to purchase the new system. Details are presented later.

\section{UFMulti distributed computing software development}

The UFMulti software system we developed at UF has been the comerstone of our computing effort since 1988. With it a single HEP application can be distributed across a large set of Unix machines and run in parallel. ${ }^{1-4}$ This has allowed us to take advantage of the microprocessor revolution to acquire a large amount of computing power at low cost, because we do not need the expensive high bandwidth buses used in almost all parallel processor designs. Our development of UFMulti also helps generate funding for computer resources and enables us to negotiate better discounts from computer vendors.

Starting in Sept. 1991, we redesigned UFMulti from the ground up, paying attention to issues of particular importance to high energy physicists such as network throughput, parallelization of $\mathrm{CPU}$ and $\mathrm{I} / \mathrm{O}$, fault tolerance and histogram/statistics collection. The package is portable across several versions of Unix and requires minimal extra coding to implement in existing HEP experiments. The work was done in collaboration with Prof. Theodore Johnson of the Computer Science Department and his students John Brothers, Krishna Harathi, Jagadeesh Kasaraneni and Aric Zion. The people involved from Physics are Avery, Chandra Chegireddy (System Manager), and Ransom Stephens (CLEO post-doc). The software is explained at more length in the attached writeups.

Ransom Stephens is now parallelizing the CLEO Monte Carlo and Compress with UFMulti, a job that should be finished by the end of Summer, 1993. We are already using the new package for physics analysis.

We have received some recognition for this work. Avery was a member of two SSC Computer Planning Committees (I and II) and chaired the SSC Physics Detector Simulation Facility (PDSF) Review Panel in Oct. 1992. He has given talks on UFMulti at ICHEP92 in Dallas and DPF92 in Chicago (see attached writeups). He has given colloquia at LSU (May 1992) and in the Computer Science Department at UF (April 1992) in addition to many seminars.

\section{Present system configuration}

The total usable computing capacity of our Unix farm is roughly 500 Vax equivalents (420 in the compute server and 90 on desktops), when the I/O rate is not saturated. This represents the 
largest concentrated computing capability in CLEO outside Cornell. The system is made up of the following components:

Unix computers

- $\quad$ DEC 5000 disk server (48 MB) for users to $\log$ on and do routine work.

- $21 \mathrm{DEC} 5000$ 's (16 MB) comprising the Unix computing farm. 17 of these are linked by FDDI, theoretically providing a useable $\mathrm{I} / \mathrm{O}$ throughput of roughly 20 times times that of Ethernet. The location of disk resources becomes less of a crucial issue when the processors are linked by FDDI. We were the first university site in the U.S. to construct an FDDI ring.

- 6 desktop DEC 5000's (16 MB) with 16 inch color screens.

- 1 DEC 5000 and 1 DEC 3100 at Cornell used by our Cornell resident post-doc and us when we visit. A $1.35 \mathrm{~GB}$ disk and a $4 \mathrm{~mm}$ DAT drive are attatched to the DEC 5000.

- 2 NeXT computers (20 MB) used as ordinary Unix development platforms.

Vax computers

- 2 VAX 3100 model 38s (24 MB) which are used as servers for the VAX system.

- 1 VAX 3200 (8 MB) used for our HEPnet connection to Fermilab.

- 7 VAX 3100 workstations 3100 s used by the theory students.

Xterminals

- 6 NCD Xterminals used by the High Energy theorists.

PCs

- Several PCs are scattered throughout the HEP group.

Disk and tape

- 32 Gbytes of disk attached to the DEC 5000s. 29 Gbytes of this is used for scratch storage for CLEO II data and Monte Carlo simulations. The disk is distributed around the computing farm so that multiprocessing jobs using UFMulti can effectively parallelize their I/O. This is very important for CLEO physics analysis which involves several Gbytes of data being processed on each pass.

- 6 Gbytes of disk attached to the Vaxes.

- $28 \mathrm{~mm}$ Exabyte drives, $34 \mathrm{~mm}$ DAT drives and $4 \mathrm{CD}$ readers. These are split between the VAX and Unix systems. . 


\section{Wide area network connections}

We have two connections with the outside world: (1) a dedicated 9600 baud line to Florida State University which routes the connection directly to Fermilab and HEPnet and (2) a connection via several T1 lines to NSFnet maintained by the University.

\section{Current computer usage}

A large amount of analysis has been carried out on the Unix machines as described below. In addition, the experimental HEP group has taken the leadership role in departmental computing. We have provided accounts for many members of the department as a good will gesture to allow them to carry out low level activities such as word processing and e-mail. We also provide system services and software for the undergraduate physics majors who have their own DEC 5000 workstation. These activities in turn have made it easier for us to press for maintenance funds.

Our large computing facilities have enabled Florida to take on the largest computational tasks in the CLEO collaboration as well as in other areas. We greatly benefit from the fact that the entire CLEO II data sample is on disk and can be accessed remotely without tape mounts. It also makes it much easier for our colleagues collaborating with us from Cornell to work with us. Some of the more computationally intense tasks are detailed below.

\section{Recompress of CLEO data}

CLEO raw data passes through the standard data reduction step called Compress, currently handled by a set of DECstations at Cornell. However, software evolution sometimes requires that all data taken up to the current time must be recompressed. This is a massive undertaking since CLEO musi have computing resources sufficient to cover current data taking plus the reanalysis. As time goes on, each recompress becomes more difficult because of the need to redo all the data taken up to that point.

Florida has carried out the greater part of the two most recent recompresses, once in 1990 (50\% of data) and once in $1991-1992$ (75\% of data) using our DECstation facilities. These tasks required the use of 14 machines of our farm and most of the disk over a 5 month period. Ransom Stephens and Jorge Rodriguez did a heroic job of getting this data processed and our overall effort was widely recognized within CLEO.

\section{Data analysis}

Most of our physics analyses are being done on the UF system. These include Yelton's charmed baryon analyses, Avery, Rodriguez and Browder's search for color suppressed B decay channels, Ransom Stephens' search for hadronic $b \rightarrow u$ modes involving the $D_{s}$ and Arne Freyberger's charm semileptonic analysis. 


\section{CLEO Monte Carlo}

The above analyses all heavily used CPU and disk resources in generating GEANT Monte Carlo samples. We are also generating about $25 \%$ of the generic Monte Carlo sample used by the entire collaboration.

\section{SDC Monte Carlo}

Rick Field and his post-doc Paul Griffin have been performing simulations for SDC. As a member of the forward calorimeter subgroup of SDC, he has been studying the production and identification of Higgs particles in SSC collisions. As a result of this work Griffin was named an SSC Fellow in 1993.

\section{Supersymmetry study}

Pierre Ramond and three of his students carried out a massive Supersymmetry analysis in which the strong, weak and electromagnetic coupling constants were run out to energies of $10^{16}$ $\mathrm{GeV}$. Several hundred runs were made on our system for this study, which was submitted for publication. A much more extensive effort is planned.

\section{Projected computing usage}

Our acquisition of computing equipment has been more than matched by the demands we have placed on them. This trend is accelerating, driven largely by the high rate of data collection at CLEO, which in turn increases the computing resources needed by data analysis, GEANT Monte Carlo, Compress data reduction and Recompress of all data. Recompress and Monte Carlo require by far the most computing cycles, and are the areas in which Florida has contributed most heavily. We have committed ourselves to these activities over the next few years.

Expected improvements to CESR will lead to a factor of 5 increase in luminosity by 1997 or so. Even by late 1996, before these improvements have all been made, we will have a factor of 5 more data than at present. Thus computing and storage issues are paramount. To effectively carry out the tasks described below we propose to replace our existing DEC 5000 based system with DEC Alphas. Our plan for this is described in the next section.

\section{Compress of incoming CLEO data}

Florida has agreed to provide the computing resources to carry out the ongoing Compress data reduction, about $30-50 \mathrm{pb}^{-1}$ per week. SMU will provide the personnel to run Compress and take care of tape handling and bookkeeping.

\section{Recompress of CLEO data}

Florida will redo the entire CLEO data reduction ("Recompress") starting late Fall, 1993 on the entire accumulated data sample, more than twice as large as the one we analyzed in 1992. As in the case of Compress, we provide the computing resources and parallelize the Compress code

while SMU does the tape handling and bookkeeping. We project that with 12 DEC $3000-400$ 
Alphas, we can reduce about $200 \mathrm{pb}^{-1}$ per week, so that $2.5 \mathrm{fb}^{-1}$ of data will take about $3-4$ months to complete, depending on how much data CLEO accumulates during that time. This represents a large improvement over the previous 5 months time to analyze a smaller $\left(1.2 \mathrm{fb}^{-1}\right)$ dataset.

We will need more powerful CPU resources in order to finish the job in a reasonable time period (not more than a few months). Several hundred $4 \mathrm{~mm}$ and $8 \mathrm{~mm}$ tapes must be processed, and output DSTs must be written. Our plan is to use UFMulti to simplify the processing by going tape to tape and avoid the large storage overhead incurred when data sets are split on disk and recombined later. The bookkeeping will also be simplified since if there is a problem running a particular job, the tapes can be rewound and the job started again without slowing down the rest of the system.

From experience we expect that Recompress will happen about every 18 months or so, driven mostly by ongoing improvements in charged particle tracking, shower fitting and particle $\mathrm{DD}$. With the current rate of data collection at CLEO (about $2 \mathrm{fb}^{-1}$ per year), Recompress will demand an ever increasing share of resources.

\section{CLEO Monte Carlo and analysis}

With the new Alphas we project that Florida will be able to generate a minimum of $75 \%$ of the generic Monte Carlo sample used by all of CLEO. We can generate about 3 million events per month on 12 DEC $3000-400$ s and 5 DEC 3000-300 desktops, assuming that $50 \%$ of the computing resources are devoted to it (this is a reasonable percentage considering our other commitments). In general, the number of required Monte Carlo events follows the total number of events in the data sample.

With the much larger data samples that will be stored on disk, our physics analyses and GEANT Monte Carlo simulations will require more computing power and disk space. These include the processes we are studying now, such as $B \rightarrow D_{s}^{(*)} X$, color suppressed $B$ modes, charmed baryon processes and charm decays. CLEO members who are collaborating with us on these projects will also be pursuing related analyses and simulations on our system.

\section{SDC simulations}

Rick Field and his post-doc Paul Griffin will do more SDC simulations of Higgs particle production. Avery will be performing tracking simulations as well, starting in 1994.

\section{Supersymmetry program}

Pierre Ramond and his students will be extending their previous work with a much more ambitious program to explore new regions of parameter space of the Supersymmetric extension to the Standard Model. The new program will require another order of magnitude increase in computing use. 


\section{New computing initiative}

We have undertaken a new computing initiative with several other universities (Cornell, UCSD, Carleton) and industry groups (Xerox, DEC). A primary goal of the project is to meet the long term computing needs of CLEO through a sophisticated and efficient use of distributed computing resources over wide areas. Since the membership of CLEO is distributed across North America, the need for computing resources and remote participation can both be served by widearea distributed computing.

The collaboration is somewhat unique in that high energy physicists and computer scientists will be working closely together to develop fault tolerant distributed computing over many, even hundreds, of computers distributed either locally or over geographically dispersed areas. The initiative will use CLEO data to provide a real world laboratory for the ideas developed by our computer science colleagues. The Cornell Isis and Meta distributed computing projects and our UFMulti effort will be merged into this new activity.

A goal of the initiative is to take advantage of ATM (Asynchronous Transfer Mode) as soon as possible so as to naturally evolve our computing resources to use the NREN "information highway" proposed by the current Administration. ATM is the network technology that will most likely provide the underpinnings for the NREN. We will set up three computing testbed sites, at Florida, Comell and Carleton. Eventually these will be linked by ATM to form a geographically dispersed computing system.

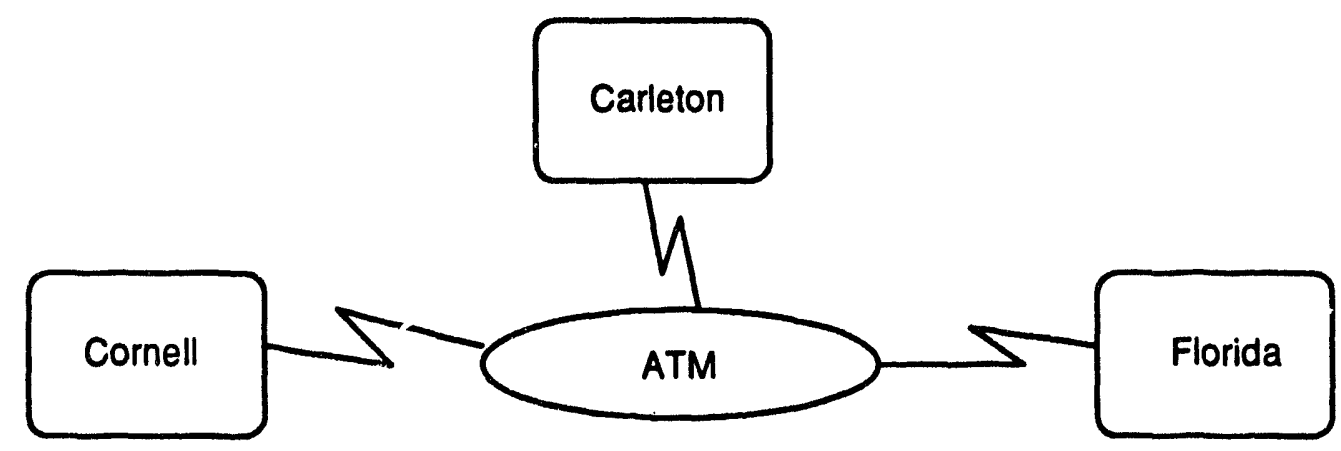

Transcontinental data analysis in our ATM based HPCC proposal

We submitted on June 14 a proposal to the High Performance Computing and Communications (HPCC) program at NSF to turn the upgrade into an effective supercomputer by adding a very fast network switch and lots of disk. Proposals to other agencies will be made as necessary.

\section{Computing upgrade plan}

We propose to upgrade our DEC 5000 computer system to one based on the new DEC Alpha processors using funding resources at Florida, DEC and DOE. Although the upgrade can be justified solely on the basis of needed CPU capacity, we will use it as a first step in our new computing initiative. 


\section{Upgrade plan (Year 1)}

The most important part of the upgrade is in Year 1 where we are asking for about $\$ 50 \mathrm{~K}$ of DOE funds in addition to those we normally get for routine upgrading, maintenance and support. We propose to purchase 24 DEC 3000-400 (64MB) Alpha servers and 5 DEC 3000-300 (64 MB) desktops to satisfy the most immediate computing needs. 12 of the servers will be deployed at Florida and the other 12 will be placed at Cornell for CLEO data reduction (Compress and Recompress). Note that this step is independent of HPCC and our computing initiative. We propose the following funding scheme:

UF $\quad \$ 150 \mathrm{~K}$ (already obtained)

DOE $\quad 50 \mathrm{~K}$

Total $\$ 200 \mathrm{~K}$

We have secured $75 \%$ of the total cost and we are requesting DOE for the remaining $25 \%$. We have a negotiated a special deal with DEC (based DEC's participation in the initiative) which allows us to purchase Alpha workstations at a 75\% discount off list price over the next few months. We are also negotiating jointly with Cornell an External Research Proposal (ERP) with DEC which allows us to buy all equipment at a 75\% discount off list price. The ERP is also based on the new computing initiative and obviously gives us tremendous leverage for any funds we acquire. The ERP has not been signed yet but we have had two previous ERP agreements and expect negotiations to be completed by the end of Summer, 1993.

Future upgrades (Years 2-3)

In these years we would not ask DOE for additional DEC Alphas and would go back to our normal funding level.

\section{Step 2 (HPCC funds)}

We will use HPCC funds to purchase additional disk and a DEC Gigaswitch at each site to link the compute servers by a high speed network. The ERP will be used here to maximize our funding leverage. The Gigaswitch is an FDDI crossbar switch which permits high speed I/O between computers connected to it. If the HPCC is not approved we will buy a somewhat reduced system.

\section{Step 3 (HPCC funds)}

Use HPCC funds in the second year to purchase an ATM switch and cards that will directly connect the Alphas to it. This will give us our first attempt at communicating with ATM locally. Our strategy is to take advantage of the NREN as it evolves to develop wide area computing. Initially we will probably use campus wide or metropolitan ATM to explore issues of latency, I/O bandwidth, etc. on distributed system performance. 
Cornell configuration

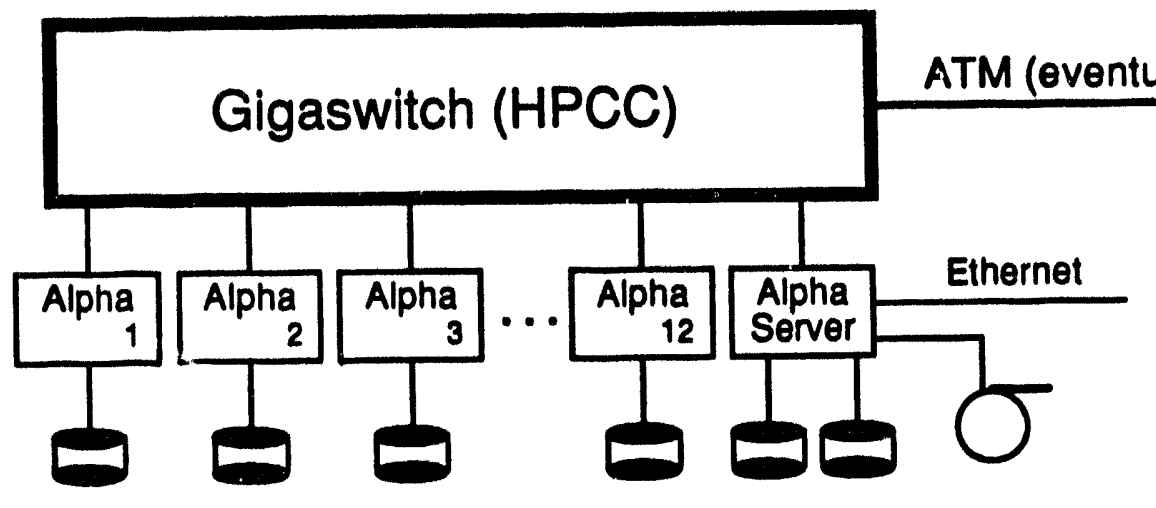

Florida configuration

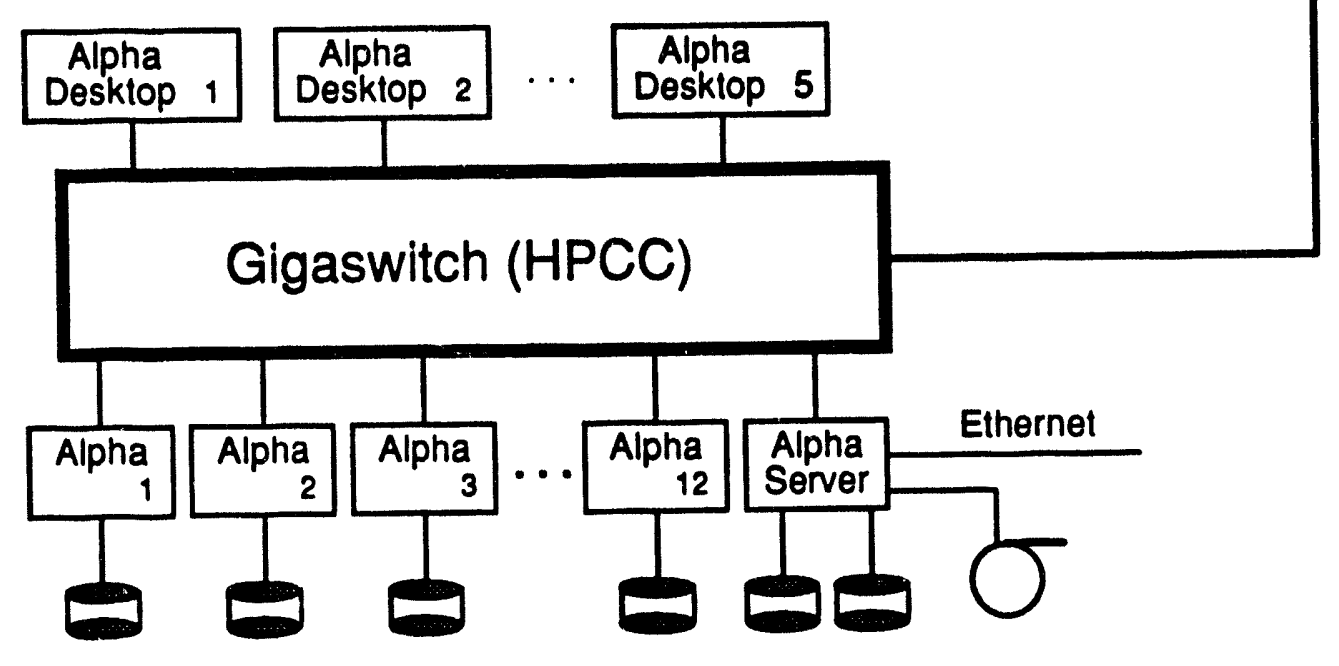


References

1. Paul Avery and Andrew White, "UFMulti: a New Parallel Processing Software System for HEP", in Proceedings of the International Conference in High Energy Physics, Ed. R.C.E. Devenish, North Holland, Oxford, England, 1989.

2. Andrew White and Paul Avery, "UFMulti: a New Parallel Processing Software System for HEP", in Proceedings of the International Conference in High Energy Physics, Santa Fe, NM, 1990.

3. Paul Avery, "A New Approach to Distributed Computing in High Energy Physics", in Proceedings of the XXVI International Conference on High Energy Physics, Ed. James Sanford, American Institute of Physics, Dallas, TX., August, 1992.

4. Paul Avery, "Distributed HEP Computing with NetQueues", in Proceedings of the 7th Meeting of the American Physical Society Division of Particles and Fields, Ed. Carl Albright, World Scientific, Chicago, Il., November, 1992.

Date END

$9 / 27 / 94$ 